\title{
The multilayer architecture of the global input-output network and its properties
}

\author{
Paolo Bartesaghi ${ }^{\mathrm{a}}$, Gian Paolo Clemente ${ }^{\mathrm{b}}$, Rosanna Grassi ${ }^{\mathrm{a}, *}$, Duc Thi Luu ${ }^{\mathrm{c}}$ \\ ${ }^{a}$ University of Milano - Bicocca, Via Bicocca degli Arcimboldi 8, 20126 Milano, Italy \\ email: paolo.bartesaghi@unimib.it; rosanna.grassi@unimib.it \\ ${ }^{b}$ Università Cattolica del Sacro Cuore di Milano, Largo Gemelli 1, 20123 Milano, Italy \\ email: gianpaolo.clemente@unicatt.it \\ ${ }^{c}$ Department of Economics, University of Kiel, Germany \\ email:d.t.luu@economics.uni-kiel.de
}

\begin{abstract}
We analyze the multilayer architecture of the global input-output network using sectoral trade data (WIOD, 2016 release). With a focus on the mesoscale structure and related properties, our multilayer analysis takes into consideration the splitting into industry-based layers in order to catch more peculiar relationships between countries that cannot be detected from the analysis of the single-layer aggregated network.

We can identify several large international communities in which some countries trade more intensively in some specific layers. However, interestingly, our results show that these clusters can restructure and evolve over time. In general, not only their internal composition changes, but the centrality rankings of the members inside are also reordered, industries from some countries diminishing their role and others from other countries growing importance. These changes in the large international clusters may reflect the outcomes and the dynamics of cooperation, partner selection and competition among industries and among countries in the global input-output network.
\end{abstract}

Keywords: Input-output linkages, Global trade, International trade clusters, Mesoscale structure, Multilayer architecture, Layer-layer interdependencies.

JEL CLASSIFICATION: C67, F10, F40

\section{Introduction}

The network architecture plays a central role in explaining the propagation of shocks and the robustness of different financial and economic systems. The 2007-2009 global recession and the subsequent European debt crisis have shown that the distress of some financial institutions in a particular country could be easily transmitted across borders into other countries, because of international inter-dependencies and the very high degree of financial integration (see, e.g., Cetorelli \& Goldberg, 2011; Shin, 2012, Kleimeier et al., 2013, Bostandzic \& Weiß, 2018, Hale et al., 2019, Park \& Shin, 2020). From the sphere of real economic activities, in an economic system of heterogeneous and interdependent agents (e.g., industries or firms), the understanding of the structure of their interactions plays a crucial role in exploring how shocks of a specific agent can be propagated to the others possibly leading to a large aggregate fluctuation or a systemic failure in the economy

\footnotetext{
${ }^{*}$ Corresponding author. email: rosanna.grassi@unimib.it
} 
(see, e.g., Acemoglu et al., 2012, Carvalho, 2014, Acemoglu et al., 2016, 2017, Atalay, 2017, Eppinger et al., 2020). It has been also suggested that such understanding at an international scale is useful in examining the cross-country transmission and the propagation of local shocks (see, e.g., Carvalho et al., 2016; Acemoglu et al., 2016; Luu et al., 2018a b; Boehm et al., 2019, Eppinger et al., 2020, Giammetti et al., 2020, among others). In summary, through input-output linkages in a production network, shocks to a particular node may have two potential effects on other nodes: (i) supply-side shocks generate propagation to the downstream customers, and (ii) demand-side shocks generate effects on upstream suppliers (e.g. see Acemoglu et al. 2016). Furthermore, direct and indirect propagations capture both the first-order impact on the "nearest neighbors" of the affected industry as well as the higher-order impacts on those who are the customers of the customers or the suppliers of the suppliers, and so on. As shown in Acemoglu et al. (2012), not only lowerorder structural properties among sectors, but also more complex properties of the economic network can play a defining role in explaining cascade effects in the whole system. More specifically, even if two different networks have the same first-order characteristics (e.g. same degree or strength sequence) different higherorder interactions among industries may lead to different severity levels of aggregate downturns Acemoglu et al. 2012) 1

More in general, once the network structure is taken into consideration, the understanding of topological properties, from a microscopic to a macroscopic perspective, is crucial to assess how a local shock or disruption to a specific node or cluster of nodes can be propagated and amplified to the rest of the system. This is why the understanding of the higher-order topological properties (such as those at the mesoscale level like the community structure studied in the present work) may have meaningful implications in managing complex economic systems. In contrast to homogeneous random and regular graphs, it is often observed that real networks properties are more heterogeneous (e.g. see Newman, 2003). For example, some nodes may have intensive interactions with many other nodes while some others only concentrate on few partners (heterogeneity in the degree and strength sequences). Furthermore, at a broader scale, nodes and edges may fall into different sets such that the internal interactions within these groups are stronger than those between different ones (heterogeneity in clustering behaviors). The latter feature is often referred to as the community structure, which has been intensively studied in the literature for a wide range of networked systems (e.g. see Fortunato, 2010, and the literature therein for the detection of this structure in various networks from different fields).

From a perspective of policy implications, the identification of such a mesoscale structure can be successfully utilized to assess the functioning, the stability and the evolution of networks. Let us consider an economic system of interacting agents as an example. First, if agents can be classified into different blocks, one could examine whether a local shock or a "communication" will spend most of the time or will even be trapped in a particular block or will be spread widely into the whole system. However, in the presence of large blocks (e.g. referring to the present work, large international trading communities), the diffusion of a shock may have a pervasive effect on many other internal nodes.

Moreover, further analysis of the importance and position of nodes within each of these groups may also

\footnotetext{
${ }^{1}$ Different higher-order of connectivity coefficients can be defined to capture more complex patterns of cascades in an inputoutput network. For example, at the third-order level of inter-connection, higher aggregate fluctuations could be observed if in the network the sectors having high strengths also share many common suppliers (see Acemoglu et al. 2012, for further details).
} 
provide meaningful implications, e.g. (i) central nodes that have many links to the other partners may play a crucial role in the functioning of that group, and (ii) boundary nodes that are mutually connected with those from different blocks may play an important role in blocks' communication and act as the "gatekeepers" or "middle men" that spread shocks and carry on them from a block to another one.

On top of this, such an identification can be used to explore the network origin of the business cycle comovements among sectors or countries, since agents who belong to a tightly connected cluster (or community) may tend to synchronize more their economic activities (e.g. see Long \& Plosser, 1983, Burstein et al., 2008. Di Giovanni \& Levchenko, 2010, Johnson, 2014, Di Giovanni et al., 2018, among others). For example, in a micro-founded model of business cycles, Long \& Plosser (1983) show that input-output interdependencies could be an important factor responsible for the co-movements among the outputs of different industries in the economy. At the international scale, Burstein et al. (2008) suggest that countries more engaged in sharing international productions exhibit a higher level of co-movement of business cycles. In another study, Di Giovanni \& Levchenko (2010) find that nations with strongly trading relations among themselves also often show a higher level of business cycle synchronization. In a similar vein, from a micro perspective, Di Giovanni et al. (2018) report that French firms, that have stronger trade linkages with a particular foreign country, also tend to have with it a higher correlation. Among different observed economic networks, perhaps the global trade system has received a more remarkable attention, due to its economic importance and data availability. It has been widely suggested that the world trade system exhibits a heterogeneous structure at different orders of interconnection. For example, using the methods of network analysis and analyzing the network of merchandise trade imbalances between countries over the period from 1948 to 2000, Serrano et al. (2007) find the presence of both local heterogeneity (when only few trade linkages possess a large percentage of the country's total flow) and global heterogeneity (when only a small proportion of all the trade linkages in the whole network carries most of its total flow). Fagiolo et al. (2008), carrying out a weighted network analysis over the period 1981-2000, show that many weak trade linkages coexist with some much stronger ones, and countries with intense trade relationships tend to be more clustered together. De Benedictis \& Tajoli (2011) analyze the World Trade Network (WTN) over the years from 1950 to 2000 and confirm the presence of trading blocks with a strong heterogeneity in the way countries select their trade partners. Focusing on the mesoscale structure of the WTN, Bartesaghi et al. (2020) show that the global trade system can be decomposed into different communities in which the internal interactions among members in each group are more intensive than the external ones. In addition, within each group, there exist some "main, central" countries "surrounded" by the other "satellite" members. The persistence of communities in the global trade system has been also investigated during the COVID-19 pandemic, focusing on centrality measures that are meaningful in capturing possible structural modifications Antonietti et al. 2022). Furthermore, although the global trade system has a certain degree of persistence over a definite time horizon, if we investigate deeper the temporal dynamics of different structural properties of the network, we can observe an evolution over time (e.g., see Serrano et al. 2007, De Benedictis \& Tajoli, 2011). The driving forces of such temporal dynamics can be traced back to several concepts and factors suggested by trade theories. For example, due to the extensive margin effects, the system can expand if new trade linkages are established. In contrast, with the intensive margin effects, the network dynamics can be driven by changes 
in the strength of the trade relationships over time (e.g., c.f. Felbermayr \& Kohler, 2006, Helpman et al. 2008, and the literature therein). There could be other factors governing the evolution of the global trade system, such as changes in the country fitness (e.g., Anderson, 1979, Garlaschelli \& Loffredo, 2005), new partnerships of bilateral and multi-bilateral trade agreements or currency unions (e.g., Subramanian \& Wei, 2007; Helpman et al., 2008; De Benedictis \& Tajoli, 2011). Mundt (2021) proposes an empirical model of network formation that takes into account the endogeneity of structural network characteristics. Applied to the Input-Output data, the model shows significant fluctuations of network ties over time, inducing changes also in the aggregate network structural properties. While much of empirical analysis of economic networks has so far either focused on single types of relations separately or combined all of them together into an aggregated one, less attention has been paid to a much richer structure of these networks, i.e. the so-called multilayer architecture (e.g. Bianconi, 2018; Battiston et al. 2018). In fact, in many real networks, various types of interactions may coexist: linkages among different nodes of the same type, linkages among different nodes from different types, and those between nodes with their copies (replicas). For example, in the financial system, banks often interact among themselves in different layers via different channels and various markets (e.g. see Leon et al., 2014; Poledna et al., 2015; Bargigli et al., 2015; Luu \& Lux, 2019). In a similar vein, the global trade system exhibits a multilayer structure, which should be considered as one of the critical factors for the emergence of multiple channels of cross-border propagation of economic crises (e.g. Lee \& Goh, 2016). In the increasing integration of global economies, each country may simultaneously trade with other countries on many different commodities-based layers (e.g. see Barigozzi et al., 2010, Gemmetto \& Garlaschelli, 2014. Gemmetto et al., 2016). Barigozzi et al. (2010) study the properties of the multilayer trade network where each of 97 layers represents trade relations among 162 countries in a particular commodity over the 19922003 period. Interestingly, their findings suggest that the distributions of link-weights (trade intensities), the averages of connectivity, the clustering coefficients and centrality levels vary across commodity-based layers and are very different from those of the aggregated trade network. The heterogeneity and dissimilarity among layers may reflect the increasing specialization process in the global trade network when countries tend to focus on certain products. Gemmetto \& Garlaschelli (2014) and Gemmetto et al. (2016) apply a statistical network approach to examine the role of the intrinsic heterogeneity in the local constraints like the degree sequences and/or strength sequences in explaining the overlaps and correlations among layers in the undirected and the directed versions of the international trade network. The network is comprised of more than 200 nodes (countries) with nearly 100 layers representing different traded commodities. Comparing across layers (traded commodities), the weighted analysis shows that while many pairs of layers display only a negligible degree of similarities between themselves, few layers exhibit a high level of overlaps, implying that some countries tend to export or import more with a similar set of trading partners in some particular commodities. These studies also suggest an important role of the distribution of hubs (i.e., countries with a high level network centrality) in explaining the relations between layers.

Empirical analyses, with a focus on the global production system that comprised supply-chain or inputoutput trade linkages among industries from different countries, have been received less attention due to fact that more comprehensive, global data only becomes available only recently. As pointed out in Baldwin \& Lopez-Gonzalez (2015), international production relations have been flourishing over the last few decades and

\footnotetext{
${ }^{2}$ In this case, the binary links already exist but the weights can become more (or less) intensive over time.
} 
they still continue to evolve. The authors also suggest that more work is needed to uncover the complexity and hidden patterns embedded inside the global production system, as the importance and the degree of integration or dependency varies across countries, sectors, types of intermediate and final goods in such system. To our best knowledge, one of the first attempts to analyze the topological properties of the world input-output network of the individual industries from different countries was the study of Cerina et al. (2015). Using the World Input Output Database (WIOD, 2013 release), Cerina et al. (2015) study different properties from the local to the global level of the world input-output network where nodes are the individual sectors while weighted links represent the monetary trading flows between them. Their results suggest that, overall, the binary links are very dense but the interaction intensities are highly asymmetric. At the local level, the authors show that the network analysis, based on different centrality and community coreness measures, can provide deeper insights into identification of the key sectors. In a recent work, focusing on the production network among industries from 20 country members in the European Monetary Union (EMU), Luu et al. (2018a) show that the external (cross-border) input-output linkages exhibit a hierarchical structure in which a small number of industries from few country members trade more intensely among themselves and form a cohesive core. In contrast, less active "satellite" industries mostly trade with the key sectors in the core. From a network perspective, these key sectors, indeed, play as hubs bridging industries from different country members of the EMU. As shown in Luu et al. (2018b), such an asymmetric network architecture has crucial implications for the understanding of the pathway on how a local shock to a particular sector in a country member of the EMU can be transmitted to those in other countries. Using the methods of the multilayer analysis, Russo et al. (2022) investigate the evolution of the international trading network of different components and parts of the automotive industry over the period 1993-2018. According to their study, several denser and more internally cohesive sub-networks can be identified like a cluster comprised by trade relations of Germany with some Central Eastern European countries, another cluster formed by the US and its partners in the preferential trade agreements such as Canada and Mexico, and another one with a prominent role of Japan and China. Russo et al. (2022) also show that the shape as well as the composition of clusters and the relative importance of countries also change over the years, with a remarkable rise of China after its accession to WTO.

The better understanding of the production networks can, of course, be useful for conducting effective industrial policies. For instance, successful experience and lessons from Japan, South Korea, Taiwan, and other "Newly Industrialized Country" (NIC) have pointed out that industrial policies, that select some "strategic" sectors to promote, can play a prominent role in facilitating the industrialization process (e.g., see Ciccone, 2002, Pack \& Saggi, 2006, Liu, 2019, Antràs \& de Gortari, 2020, among others). However, under the expansion of the global production network in which we often observe such a hierarchical structure dominated by few countries and their industries, the potential role of industrial policies (especially for less developed countries or for infant industries) might be more limited than what was traditionally supposed (Pack \& Saggi, 2006).

The main contribution of our study is to provide an analysis of the global trade network from a different perspective, i.e. the multilayer architecture of the input-output interdependencies among industries and countries. In our multilayer network countries are nodes and sectors represent layers and we focus on the mesoscale structure and related network properties spanning from nodes to nodes across layers. We consider a comprehensive structure of the network in which different types of connections (i.e. intralayer as well as interlayer ones) do exist all together. In addition, after classifying different clusters of nodes and associated 
layers, we further investigate the internal structural properties of each cluster and then compare with those of the other clusters. We also examine the role of individual countries and industries in forming and functioning different large international clusters. Through these analyses, we aim to uncover hidden structures and complexities inside the detected communities and shed light on the diversification and specialization patterns of countries when they trade their inputs and outputs in and between different industries-based layers. To these ends, we extend various network metrics and community detection methods used for single layer networks to those applicable for complex networks with a much richer multilayer architecture. In the next steps, we apply them to analyse the input-output relations between different sectors in different countries, using the world input-output database (WIOD, 2016 release; see Timmer et al. (2015, 2016)).

Our results show that the multilayer analysis is able to catch more peculiar trade relationships that cannot be detected at the mono-layer aggregate trade network. Among others, we find that, in the weighted version, while many layers have dissimilar internal structures, few others are somewhat more overlapped or correlated. On top of that, the interlayer interactions are also highly heterogeneous. Digging deeper at a broader mesoscale structure, we can identify several large international communities in which some countries trade more intensively in certain specific industry-based layers. However, it is worth to emphasize that such a mesoscale structure does evolve over time, which may reflect the competition as well as cooperation dynamics among industries and among nations. For example, in 2000, the first largest international community was comprised by sectors from the US, Japan together with several sectors from China, while the second largest cluster mainly consists of industries from the former Eastern Bloc's countries. In contrast, as for the data in 2014, we identify the first largest international community where relevant sectors of main Asian players (China, India, Japan, South Korea) together with those from Australia are clustered together. On the other hand, sectors from countries involved in the North American Free Trade Agreement (Canada, Mexico and USA) belong to the second largest one.

The remainder of this paper is organized as follows. In Section 2, we describe the multilayer representation of the global trade system as well as the proposed methodology used to analyze such a network. Section 3 summarizes preliminary analysis of data and the main results with related economic interpretation. A focus on temporal evolution is also provided. We conclude in Section 4 . Further information on the dataset and additional results are left to the Appendices.

\section{Network representation and methodology}

\subsection{Multilayer representation and fundamental metrics of the world input-output network}

We shall now introduce the general mathematical representation of a multilayer trade network. Formally, to deal with a multilayer network, one of the main approache 3 is to represent the trade interdependencies among $L$ sectors from $N$ different countries as a block matrix, with $L \times L$ blocks, each one of order $N$, called the weighted supra-adjacency matrix $W^{\text {supra }}$ with size $N_{\text {supra }} \times N_{\text {supra }}$, where $N_{\text {supra }}=N L$ Bianconi,

\footnotetext{
${ }^{3}$ An alternative approach consists in using tensors. For the sake of brevity, we refer here only to supra-adjacency representation. For further details of the tensor approach, we refer the reader to De Domenico et al. (2013) and Bartesaghi et al. (2022).
} 
2018):

$$
W^{\text {supra }}=\left[\begin{array}{cccc}
W^{[1,1]} & W^{[1,2]} & \ldots & W^{[1, L]} \\
W^{[2,1]} & W^{[2,2]} & \ldots & W^{[2, L]} \\
\vdots & \vdots & \ddots & \vdots \\
W^{[L, 1]} & W^{[L, 2]} & \ldots & W^{[L, L]}
\end{array}\right]
$$

Each $W^{[\alpha, \alpha]}$ is a $N$-square block matrix representing the intralayer interactions among $N$ nodes in layer $\alpha$, while when $\alpha \neq \beta$, each $W^{[\alpha, \beta]}$ with size $N \times N$ is a weighted (adjacency) block matrix capturing the interlayer interactions between nodes in layer $\alpha$ and nodes in layer $\beta$.

Its binary version, indicating the existence of links, is characterized by a supra-adjacency matrix $A^{\text {supra }}$ with the same size, where the block matrix $W^{[\alpha, \beta]}$ is replaced by the binary matrix $A^{[\alpha, \beta]}$.

The supra weighted matrix $W^{\text {supra }}$ can be further decomposed into two distinct parts $W^{\text {supra }}=W^{\text {intra }}+$ $W^{\text {inter }}$, in which the first part $W^{\text {intra }}$ consists of only intralayer linkages and the second part $W^{\text {inter }}$ consists of only external interlayer linkages:

$$
W^{i n t r a}=\left[\begin{array}{cccc}
W^{[1,1]} & O & \ldots & O \\
O & W^{[2,2]} & \ldots & O \\
\vdots & \vdots & \ddots & \vdots \\
O & O & \ldots & W^{[L, L]}
\end{array}\right]
$$

and

$$
W^{\text {inter }}=\left[\begin{array}{cccc}
O & W^{[1,2]} & \ldots & W^{[1, L]} \\
W^{[2,1]} & O & \ldots & W^{[2, L]} \\
\vdots & \vdots & \ddots & \vdots \\
W^{[L, 1]} & W^{[L, 2]} & \ldots & O
\end{array}\right]
$$

where $O$ is the $N$-square matrix whose elements are zero.

Similarly, in the binary version, we can also define $A^{\text {intra }}$ and $A^{\text {inter }}$ as the intralayer and interlayer adjacency matrices, respectively.

We can extend the standard vertex centrality measures in the multilayer context. Since each country or sector in the global trade network can be both the buyer and the seller at the same time, it is necessary to distinguish between the incoming and outgoing linkages with their partners. Given the matrix $W^{\text {supra }}$ showing the interaction intensities, and the matrix $A^{\text {supra }}$ representing the existent interactions, one can compute the different types of degrees and strengths of nodes (countries) across layers (sectors). We refer the reader to the Appendix A for the formal definitions of in- and out strengths (in and out-degree, respectively).

\subsection{Interrelations between layers}

We recall that $A^{[\alpha, \beta]}$ and $W^{[\alpha, \beta]}$ capture the interdependencies between nodes in layer $\alpha$ with nodes in layer $\beta$ in the binary and weighted versions, respectively. Hence, to examine how strong the interaction between two layers $\alpha$ and $\beta$ is, we measure the average connectivity and intensity based on the elements of 
these two matrices. Let us define the average link and weight between two layers $\alpha$ and $\beta$ as

$$
\left\langle a^{[\alpha, \beta]}\right\rangle=\frac{\sum_{i, j} A_{i j}^{[\alpha, \beta]}}{N^{2}}
$$

and

$$
\left\langle w^{[\alpha, \beta]}\right\rangle=\frac{\sum_{i, j} W_{i j}^{[\alpha, \beta]}}{N^{2}} .
$$

The average strength $\left\langle w^{[\alpha, \beta]}\right\rangle$ can be further normalized by $w^{*}$, that is the largest element of the weighted supra-adjacency matrix $W^{\text {supra }}$ :

$$
\left\langle w^{[\alpha, \beta]}\right\rangle_{n o r m}=\frac{\sum_{i, j} W_{i j}^{[\alpha, \beta]}}{N^{2} w^{*}},
$$

which will lead to $0 \leq\left\langle w^{[\alpha, \beta]}\right\rangle_{\text {norm }} \leq 1$.

In the following, we will briefly explain the methods used to measure the overall overlaps and correlations between layers. Following Gemmetto \& Garlaschelli (2014); Gemmetto et al. (2016); Luu \& Lux (2019), we define the overall (normalized) degree of overlaps between every pair of layers $\alpha$ and $\beta$ in the binary version as

$$
O_{b i n}^{[\alpha, \beta]}=\frac{2 \sum_{i, j} \min \left(A_{i j}^{[\alpha, \alpha]}, A_{i j}^{[\beta, \beta]}\right)}{\sum_{i, j}\left(A_{i j}^{[\alpha, \alpha]}+A_{i j}^{[\beta, \beta]}\right)} .
$$

Analogously, for the weighted version, the overall (normalized) level of overlaps between two layers $\alpha$ and $\beta$ is given by

$$
O_{w}^{[\alpha, \beta]}=\frac{2 \sum_{i, j} \min \left(W_{i j}^{[\alpha, \alpha]}, W_{i j}^{[\beta, \beta]}\right)}{\sum_{i, j}\left(W_{i j}^{[\alpha, \alpha]}+W_{i j}^{[\beta, \beta]}\right)} .
$$

It can be easily shown that $O_{b i n}^{[\alpha, \beta]}$ ranges in $[0,1]$, with $O_{b i n}^{[\alpha, \beta]}=0$ if and only if two layers $\alpha$ and $\beta$ have no overlaps at all, while $O_{b i n}^{[\alpha, \beta]}=1$ if the two adjacency matrices are identical. Similar interpretations apply to $O_{w}^{[\alpha, \beta]}$ used in the weighted version.

Alternatively, the overall degree of similarity between two layers $\alpha$ and $\beta$ could be measured by the Pearson correlation coefficient (element by element) between $A_{i j}^{[\alpha, \alpha]}$ and $A_{i j}^{[\beta, \beta]}$ or between $W_{i j}^{[\alpha, \alpha]}$ and $W_{i j}^{[\beta, \beta]}$ over all possible pairs of node indices $(i, j)$ :

$$
\begin{gathered}
R_{b i n}^{[\alpha, \beta]}=\frac{\left\langle A_{i j}^{[\alpha, \alpha]} A_{i j}^{[\beta, \beta]}\right\rangle-\left\langle A_{i j}^{[\alpha, \alpha]}\right\rangle\left\langle A_{i j}^{[\beta, \beta]}\right\rangle}{\sigma\left[A_{i j}^{[\alpha, \alpha]}\right] \sigma\left[A_{i j}^{[\beta, \beta]}\right]}, \\
R_{w}^{[\alpha, \beta]}=\frac{\left\langle W_{i j}^{[\alpha, \alpha]} W_{i j}^{[\beta, \beta]}\right\rangle-\left\langle W_{i j}^{[\alpha, \alpha]}\right\rangle\left\langle W_{i j}^{[\beta, \beta]}\right\rangle}{\sigma\left[W_{i j}^{[\alpha, \alpha]}\right] \sigma\left[W_{i j}^{[\beta, \beta]}\right]},
\end{gathered}
$$

where in general, $\langle X\rangle$ and $\sigma[X]$ are the notations for the mean and standard deviation of $X$.

It should be emphasized that while the average connectivity and the average intensity defined in Eqs. (4) and (6) are based on the interlayer linkages, the level of overlaps and the degree of similarity between two layers $\alpha$ and $\beta$ only depend on the intralayer linkages. In the context of the global input-output analyzed in our current work, the average connectivity and the average intensity for each pair of layers (sectors) $\alpha$ and 
$\beta$ indicate whether, over all different $N$ countries, these two sectors strongly interact or not. The level of overlap and of degree similarity, in contrast, returns the extent to which trade relations among $N$ countries in a particular industry $\alpha$ are also similar to trade relations among $N$ countries in another industry $\beta$.

\subsection{Multilayer communicability and its applications to community detection}

The communicability between every pair of nodes quantifies the number of all possible connection paths between them (Estrada et al. 2012). It should be emphasized that the basic network metrics such as degrees or strengths can only capture the second order structural interdependencies (from all other nodes to a node or from a node to all other nodes). Even the clustering coefficients, which are often used to analyse the clustering behaviors in complex networks (e.g. see Luu et al. (2017) and the literature therein), can explain the third order of the structural correlations among three nodes alone. In contrast, the communicability is able to catch richer information about the direct as well as indirect pathways associated with different orders of interconnectedness between nodes.

The communicability among nodes has already been used to detect the community structure in the context of monoplex networks (e.g. see Estrada \& Hatano, 2008; Bartesaghi et al., 2020). As a natural extension, the multilayer communicability indicates the number of paths through both possible intralayer and interlayer links that connect a given node in a particular layer to the other nodes in the multilayer architecture. In the context of global input-output network, communicability indicates the number of different upstream and downstream propagation channels between sectors and between countries, via both immediate and not immediate pathways. In fact, it can be somewhat related to the concept of the average propagation length often used to measure the economic distances between industries in the literature related to input-output analysis (e.g. Dietzenbacher et al., 2005, Miller \& Blair, 2009).

In the binary version, given a supra-adjacency matrix $A^{\text {supra }}$, the communicability matrix of the multilayer network is

$$
G_{b i n}=I+\frac{\left(A^{\text {supra }}\right)^{1}}{1 !}+\frac{\left(A^{\text {supra }}\right)^{2}}{2 !}+\ldots+\frac{\left(A^{\text {supra }}\right)^{k}}{k !}+\ldots=\exp \left(A^{\text {supra }}\right) .
$$

Note that $G_{b i n}$ can be further expressed in the form of a supra matrix as

$$
G_{b i n}=\exp \left(A^{\text {supra }}\right)=\left[\begin{array}{cccc}
G^{[1,1]} & G^{[1,2]} & \ldots & G^{[1, L]} \\
G^{[2,1]} & G^{[2,2]} & \ldots & G^{[2, L]} \\
\vdots & \vdots & \ddots & \vdots \\
G^{[L, 1]} & G^{[L, 2]} & \ldots & G^{[L, L]}
\end{array}\right]
$$

where in general $G^{[\alpha, \beta]}$ (size $\left.N \times N\right)$ is the matrix containing the communicability between pairs of nodes belonging to layer $\alpha$ and layer $\beta$ (Bianconi, 2018). As a special case, when $\alpha=\beta, G^{[\alpha, \alpha]}$ represents communicability among nodes within layer $\alpha$. However, it is worth to mention that $G^{[\alpha, \alpha]}$ may differ from $\exp \left(A^{[\alpha, \alpha]}\right)$ if there exist couplings among replicas and/or interlayer connections.

It is worth noting the similarity between the communicability matrix and the Leontief inverse matrix. They are both matrix functions obtained as a sum of a power series expansion. The Leontief inverse matrix is a resolvent-type matrix and unravels the technological interdependence within the productive systems in a given economy (e.g. Silva et al., 2017). Then, the Leontief inverse and the communicability matrix are 
related measures of aggregate fluctuation capacity and they differ in the way long walks in the network are penalized ${ }^{\mathbb{4}}$. While in the Leontief inverse equal weights are assigned to walks of different length, in the communicability matrix a walk of length $k$ is penalized $5^{5}$ in the sum by a factor $1 / k$ !. This choice accounts, in a more realistic way, for the fact that a shock originated in a node will have a reduced impact on the latter nodes in the production chain.

Exploiting the communicability matrix we can define suitable centrality measures for the nodes in the multilayer. In particular, in the directed version, for a node $i$ on layer $\alpha$, it is necessary to distinguish the incoming paths from all nodes in a layer $\beta$ to $i$ and the outgoing paths from $i$ to all nodes in a layer $\beta$. Hence, we define the receive (communicability) centrality $\mathrm{rc}_{i}^{[\alpha \leftarrow \beta]}$ and the broadcast (communicability) centrality $\mathrm{bc}_{i}^{[\alpha \rightarrow \beta]}$ as follows:

$$
\mathrm{rc}_{i}^{[\alpha \leftarrow \beta]}=\sum_{j=1}^{N} G_{j i}^{[\beta, \alpha]}, \quad \mathrm{bc}_{i}^{[\alpha \rightarrow \beta]}=\sum_{j=1}^{N} G_{i j}^{[\alpha, \beta]} .
$$

The total communicability centralities of node $i$ in layer $\alpha$ from/to all nodes in all $L$ layers are then given by

$$
\mathrm{rc}_{i}^{[\alpha, \text { total }]}=\sum_{\beta=1}^{L} \mathrm{rc}_{i}^{[\alpha, \beta]}, \quad \mathrm{bc}_{i}^{[\alpha, \text { total }]}=\sum_{\beta=1}^{L} \mathrm{bc}_{i}^{[\alpha, \beta]} .
$$

It is straightforward to extend the communicability matrix and the definitions of centrality measures to their weighed counterparts. In the next step, we can define the communicability matrix $G_{w}$ for the weighted version as

$$
G_{w}=I+\frac{\bar{W}^{\text {supra }}}{1 !}+\frac{\left(\bar{W}^{\text {supra }}\right)^{2}}{2 !}+\ldots+\frac{\left(\bar{W}^{\text {supra }}\right)^{k}}{k !}+\ldots=\exp \left(\bar{W}^{\text {supra }}\right) .
$$

where $\bar{W}^{\text {supra }}$ represents the weighted supra-matrix after a suitable normalization. The normalization helps to avoid the excessive influence of links with higher weights in the network as pointed out in Crofts \& Higham 2009) and Estrada (2011). The element $\bar{W}_{i j}^{[\alpha, \beta]}$ of the normalised matrix $\bar{W}^{\text {supra }}$ represents the incidence of the trade between sector $\alpha$ in economy $i$ and sector $\beta$ in economy $j$ with respect to the average volumes they trade.

Note that the communicability matrix, considering all possible walks between two nodes, accounts also for the incidence of the trade between sector $\alpha$ in $i$ and sector $\beta$ in $j$ through intermediate sectors/nodes. As for the inverse of the Leontief matrix, $G_{w}$ takes into consideration both the effects of indirect and direct trades, but communicability assigns a decreasing weight to indirect connections when their length (i.e., the length of the walk between two nodes) increases.

Based on the weighted communicability matrix $G_{w}$, one can also easily derive the weighted receive (communicability) centralities and broadcast (communicability) centralities, both for layer-layer communicability centralities and for the total ones, similar to the binary counterparts defined in Eqs. (13) and (14).

In the following, we will explain how to apply communicability centralities to identify communities in a multilayer network. We start by introducing on the network a suitable distance based on the idea of commu-

\footnotetext{
${ }^{4}$ Notice that, as for the Leontief matrix, the input-output interdependencies play a key role in the communicability matrix. Therefore, a similar interpretation based on micro-founded models as in Long \& Plosser (1983) can be further investigated

${ }^{5}$ Notice that a similar assignment of decreasing weight on walks can be given representing the influence structure induced by a Neumann series.
} 
nicability. To ensure that the introduced distance is well defined, we need to work with a symmetrized matrix. To this end, we first symmetrize the matrix $W^{\text {supra }}$ by constructing $W^{\text {symm }}=\frac{1}{2}\left(W^{\text {supra }}+\left(W^{\text {supra }}\right)^{T}\right)$. To avoid the excessive influence of links with higher weights in the network, we then normalize the new matrix $W^{\text {symm }}$ by $\bar{W}^{\text {symm }}=S^{-\frac{1}{2}} W^{\text {symm }} S^{-\frac{1}{2}}$, where $S$ is the (supra) diagonal matrix of the strengths of each node in each level. As expressed by the definition, the communicability between two nodes is a weighted sum of the number of all walks connecting the pair (see Estrada et al. (2012)). Indeed, the fact that two nodes can be connected by means of all possible walks, and not only by shortest paths, is implicit in the idea of communicability.

In general, the communicability-based distance $\xi_{i j}^{[\alpha, \beta]}$ between node $i$ in layer $\alpha$ and node $j$ in layer $\beta$ is defined as

$$
\xi_{i j}^{[\alpha, \beta]}=G_{i i}^{[\alpha, \alpha]}-2 G_{i j}^{[\alpha, \beta]}+G_{j j}^{[\beta, \beta]},
$$

where $G$ is computed as in Eq. (11) in the binary version and as in Eq. 15 in the weighted one by replacing $\bar{W}^{\text {supra }}$ with $\bar{W}^{\text {symm }}$. Let us also emphasize that we are assigning the same meaning to distances between nodes in the same layer, distances between versions of the same node in different layers, and distances between different nodes in different layers.

Following Chang et al. (2016) and Bartesaghi et al. (2020) we can compute the cohesion function $\gamma_{i j}^{[\alpha, \beta]}$, between any couple of nodes, as

$$
\gamma_{i j}^{[\alpha, \beta]}=\bar{\xi}_{i i}^{[\alpha, \alpha]}+\bar{\xi}_{j j}^{[\beta, \beta]}-\xi_{i j}^{[\alpha, \beta]}-\bar{\xi},
$$

where $\bar{\xi}_{i i}^{[\alpha, \alpha]}$ is the average communicability distance of node $i$ belonging to layer $\alpha$ from all the other nodes in all layers and $\bar{\xi}$ is the average communicability distance over the whole network.

The cohesion function $\gamma_{i j}^{[\alpha, \beta]}$ can be interpreted as a cohesion measure between the two nodes. Specifically, when positive (respectively, negative), it represents the gain (respectively, the cost) of grouping nodes $i$ in layer $\alpha$ and $j$ in layer $\beta$ in the same community of a given partition. We assume then to maximize the global cohesion function $\mathcal{Q}$ :

$$
\mathcal{Q}=\sum_{[i, \alpha],[j, \beta]} \gamma_{i j}^{[\alpha, \beta]} x_{[i, \alpha],[j, \beta]},
$$

where $x_{[i, \alpha],[j, \beta]}$ is the Kronecker delta function, which is equal to 1 if two nodes $[i, \alpha]$ and $[j, \beta]$ are in the same cluster and 0 otherwise. Hence, we obtain the best possible partition via maximization of the function $\mathcal{Q}$ defined in 18 .

More specifically, the network partition that allows to identify clusters and the consequent optimal partition are established according to the steps of the following algorithm.

1. let $\mathscr{G}$ be the original multilayer (in general, directed and weighted) network that has $L$ layers and $N$ nodes per layer, and let $W^{\text {supra }}$ be the corresponding supra weighted matrix;

2. to apply the communicability-based method, we first build the undirected weighted network associated with the symmetric supra weighted matrix defined as $W^{\text {symm }}=\frac{1}{2}\left(W^{\text {supra }}+\left(W^{\text {supra }}\right)^{T}\right)$;

3. we then build the undirected weighted network associated with the normalised weighted supra-adjacency matrix $\bar{W}^{\text {symm }}=S^{-\frac{1}{2}} W^{\text {symm }} S^{-\frac{1}{2}}$;

4. we compute the distances according to formula (16) and define the threshold interval $\left[\xi_{\min }, \xi_{\max }\right]$, where 
$\xi_{\min }$ and $\xi_{\max }$ represent the minimum and the maximum communicability distances between couples of nodes, respectively and set $\xi_{h}=\xi_{\text {min }}$ with the initial index $h=0$;

5. we define a $N L \times N L$ matrix $M_{h}^{\text {supra }}$ whose entries are 1 if $\xi_{i j}^{[\alpha, \beta]} \leq \xi_{h}$ and 0 otherwise and build the undirected unweighted network from the binary supra-adjacency matrix $M_{h}^{\text {supra }}$;

6. we select the partition $P_{h}$ given by the components of the network associated to $M_{h}^{\text {supra }}$ and compute the partition quality function $\mathcal{Q}$ in equation 18

7. we set the number of iterations $r$, compute the step increment $k=\frac{\xi_{\max }-\xi_{\min }}{r}$, set $\xi_{h}=\xi_{h-1}+k$ and $h=h+1$ and repeat steps 5-6 until $\xi_{h}=\xi_{\text {max }}$; and select the optimal partition $P_{h}^{\star}$ as the partition $P_{h}$ that provides the optimal $\mathcal{Q}$.

It is worth stressing few key points of the presented methodology. We aim at clustering nodes and layers $[i, \alpha]$ where $i$ are countries and $\alpha$ are sectors on the basis of a specific communicability distance. Our procedure is based on the selection of the optimal threshold. The value of the threshold allows indeed to disentangle the role of very tight relationships between couple of nodes. For instance, a very low threshold leads to a great number of isolated nodes, while higher values produce instead larger communities. In the proposed approach, the value of the threshold is not selected as a prior, but it is computed by the procedure assuring the optimal partition obtained from the maximization of the cohesion function $\mathcal{Q}$ defined in formula (18).

\section{Multilayer community structure in the global input-output network}

\subsection{Data}

We analyze the mesoscale structure and the related topological properties of the global trade network from the perspective of the multilayer architecture, using an updated version of the world input-output database (WIOD, 2016 release) 6 The updated version released in 2016 is the second wave of WIOD data, providing yearly information for trade in input-output among 56 different sectors in 44 countries and the aggregate of the rest-of-the-world (ROW) over the period from 2000 to 2014 (see Tables B.1 and B.2 in the Appendix B for a list of countries and sectors). In total, we have $56 \times 44=2464$ industries worldwide. Traded amounts (weights) among them are expressed in millions of dollars. We provide more detailed descriptive statistics of the weights in Table B.3 An initial analysis of the topology of the network has been developed. Since it includes standard techniques of network analysis, for the sake of brevity, we refer the reader to the Appendix C, The results naturally lead to the following related question from a topological perspective at a broader scale of the global trade system in input-output. Are there clusters of countries whose members interact stronger among themselves simultaneously in different layers associated with different industries? To answer this question, we conduct an analysis of a multilayer community structure in the global input-output network.

\subsection{Multilayer community structure in the global input-output network}

In this section, we report main results obtained by applying the procedure based on multilayer communicability defined in Section 2.3. The proposed approach has been applied directly to the multilayer network,

\footnotetext{
${ }^{6}$ The database is described in detail by Timmer et al. (2016). It is publicly available at: http://www.wiod.org/database/wiots16.
} 
which has been preliminarily transformed in an undirected one by substituting each pair of bilateral directed links with an undirected link, with a weight equal to the average weight.

Starting from a supra-adjacency matrix with 2464 nodes, given by 44 countries and 56 sectors and using volumes of trade in 2014, the methodology provides 117 communities (except some isolated nodes). Notice that clustered groups of nodes are located both intralayers and interlayers. This means that we can find countries that are members of the same community in a specific sector, as well as countries sharing the same community in more sectors. In particular, two large international communities (namely Community 1 and Community 2) are detected, with 443 and 318 nodes, respectively. The other ten communities have instead a smaller size, being between 30 and 50 nodes. A graphical representation of all members of the top twelve communities, in terms of number of nodes, can be found in Figure D.1 Appendix D.

Looking at the clusters obtained, some noticeable elements can be identified. First of all, we observe an Australian-Asian community, where all relevant sectors of main Asian players (China, India, Japan, South Korea) are clustered together. On the other hand, countries involved in the North American Free Trade Agreement (Canada, Mexico and USA) belong to the second community.

Furthermore, it is interesting to explore the clustering behavior of European countries that are "in the middle" between these two relevant communities. Indeed, we find that almost all of them are clustered in Community 1 for some sectors and in Community 2 for other sectors (see, for instance, Germany and Great Britain). To further explain this result, we compute for each country the number of sectors that belong to these two communities. We also compute 7 the Gini heterogeneity index 8 to give an indication of the dispersion of sectors for each country along the communities. A higher value of this index for a particular country means that, overall, its sectors are more spread out between different communities.

As regard to European countries, we notice how some of them (Bulgaria, Germany, Greece, Norway, Portugal) show a behavior similar to the Australian-Asian countries, being these countries concentrated in the first community for most trade of sectors. However, looking at the sectors, this participation occurs with a different degree of heterogeneity, as indicated by the Gini index. For instance, Germany has 37 sectors in Community 1 and only 6 sectors in Community 2. In contrast, Czech Republic, France, Great Britain, Ireland, Poland, Russia are instead more concentrated in Community 2. The remaining European countries have only a limited number of sectors belonging to the top two communities, while the other sectors are concentrated in specific groups.

Moreover, it is worth pointing out the presence of countries with a lower level of heterogeneity in the community location of their sectors, characterized by a concentration in the same community for almost all sectors (except isolated ones). Indeed, we observe that besides the top largest international clusters, the next large communities are mainly formed by different sectors of the same country. These are, for example, the cases of Spain (Community 3), Italy (Community 4), Brazil (Community 5), Finland (Community 6), Croatia (Community 7), Turkey (Community 8), Indonesia (Community 9). This behavior can be explained by stronger domestic trades with respect to relations with sectors of other countries.

The presence of large international communities together with that of smaller ones leaning to domestic or

\footnotetext{
${ }^{7}$ Detailed results are reported in Table D.1 in Appendix D

${ }^{8}$ In particular, since we deal with nominal variables, we compute the index $H_{j}$ to quantify the heterogeneity of a country $j$ as $H_{j}=1-\sum_{i=1}^{c} p_{i, j}^{2}$ where $c$ is the total number of clusters detected by the procedure explained in Section 2.3 and $p_{i, j}$ is the proportion of sectors of country $j$ in the cluster $i$ such that $\sum_{i=1}^{c} p_{i, j}=1$. This formula is also known in the literature as the Gini-Simpson index.
} 
regional input-output relations have been also found in the other studies such as Baldwin \& Lopez-Gonzalez (2015), Cerina et al. (2015), Luu et al. (2018b), Luu et al. (2018a). Indeed, industries of different countries seem to have different degrees of internationalization. Some of them have been more engaged in cross-border trade linkages and, hence, can contribute to or even lead the functioning of regional or international clusters. It should be emphasized that this is not necessary related to the size of the country (or the size of domestic market), as we do observe that the largest international communities are comprised of both sectors from large economies like the US and China and those from smaller ones like Taiwan, Ireland or Czech Republic and ROW.9 Among possible explanations, perhaps the relations between sectors from "headquarter economies" and sectors from "factory economies" play a prominent role (e.g., see Baldwin, 2006, Baldwin \& Lopez-Gonzalez, 2015). In line with the findings by De Benedictis \& Tajoli (2011) for the World Trade network, our conjecture is that the selection of the trading partners does matters for input-output relations. As discussed in Baldwin \& Lopez-Gonzalez (2015), "headquarter economies" with more advanced technology and higher wages (e.g., the US, Japan, Germany) tend to offshore certain stages of production to low-wage nations (the factory economies like Mexico, Poland and Czech Republic, Taiwan), which may lead to the creation of regional or international input-output chains across the world such as "Factory Asia", "Factory North America" and "Factory Europe". In between these two distinct types of countries, the "hybrid economies" can simultaneously play a certain role as a headquarter economy and a certain role as a factory one.

Furthermore, some industries from "headquarter economies" act as hubs with many linkages with different industries from different nations in the global input-output network. In contrast, those from "factory economies" tend to rely more on few key players from "headquarter economies". This is also, indeed, inline with the results from basic network analysis in the Appendix C, where we find in the weighted version of the network that some nodes have a much higher level of the incoming and outgoing strengths.

Now, if we look at each single sector-based layer, we can further explore how countries in the same layer are classified in communitie:10. Overall, we find that the analysis for sectors shows a higher level of heterogeneity (as shown by the Gini index), compared to that for countries. This implies that, in the same considered layer (industry), countries are on average split in several different communities. However, except for sectors "T" and "U" where countries act almost always as isolated communities, the highest number of countries in each sector belongs to one of the two largest international communities. It is also interesting to note that sectors with a lower heterogeneity (as "Mining and quarrying (B)" and "Manufacture of coke and refined petroleum products (C19)") are characterized by a concentration of countries in Community 1.

To explore the similarity between sectors, we computed the Jaccard similarity index (see Figure 3.1), displaying only coefficients higher than 0.7. In this way, we emphasize couple of sectors characterized by a similar classification of countries in communities. We observe a highest similarity between the sectors "Crop and animal production, hunting and related activities (A01)" and "Manufacture of food products, beverages and tobacco products (C10-C12)". We have indeed that all countries (except Ireland) have been classified in the same way in these two sectors. This result can be easily explained by the fact that activities involved in these two sectors are closely related (see also the analysis of the interrelations between layer-based sectors

\footnotetext{
${ }^{9}$ Note that ROW consists of different nations not listed in the database, in which important members are, for example, those of OPEC nations and ASEAN 6.

${ }^{10}$ Detailed results can be found in Table D.2 Appendix D
} 
in the Appendix C). Another interesting case is represented by the sectors F and L68 (Construction and Real Estate) that show 41 countries analogously classified (except Malta, Mexico and Ireland). The same behavior is also observed for the other two couples G47-L68 (Retail Trade - Real Estate) and G47-K64 (Retail Trade - Financial Service).

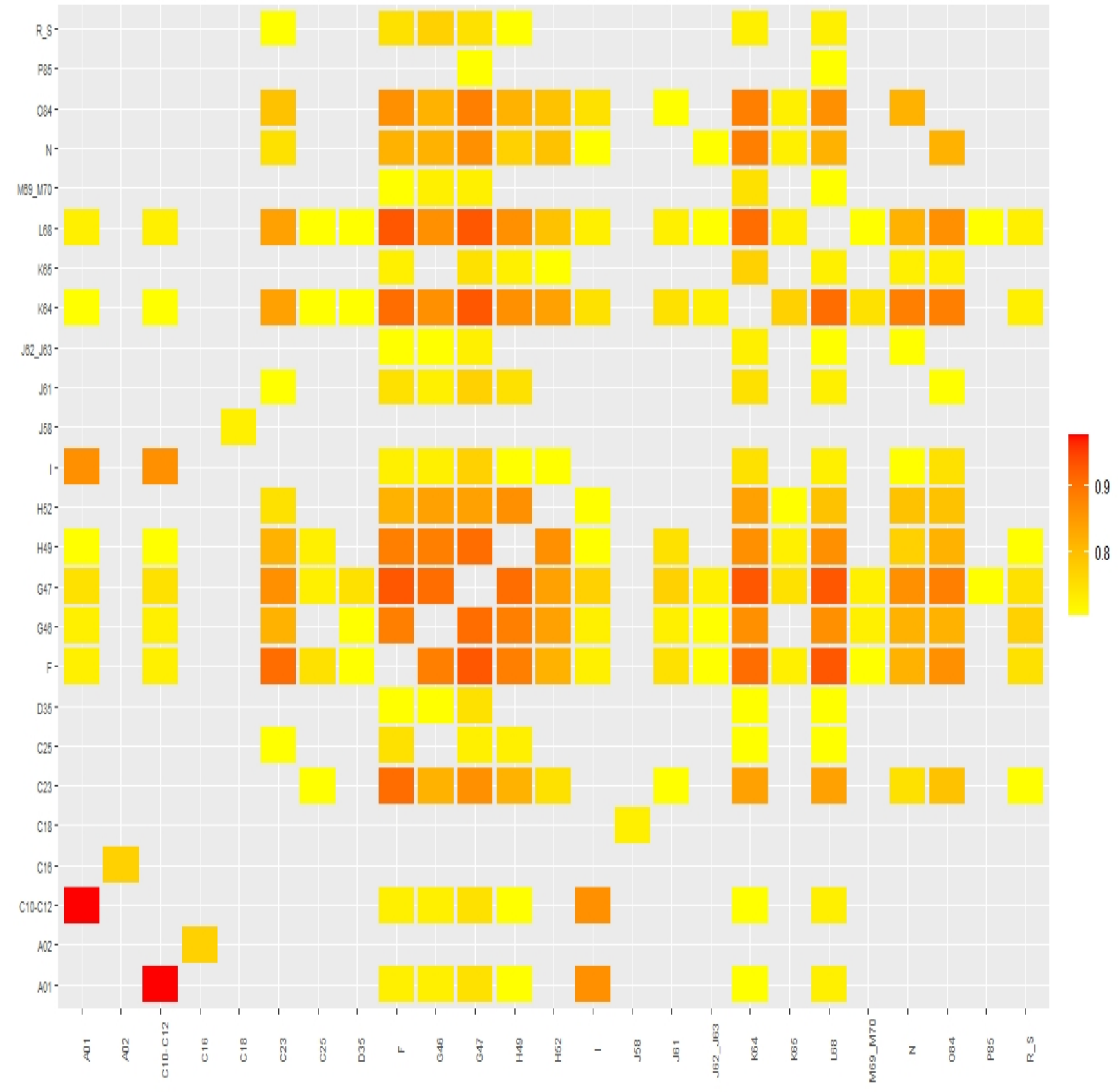

Figure 3.1: Matrix of Jaccard similarity coefficients between sectors. Only coefficients higher or equal than 0.7 have been reported (it corresponds to $90 \%$ quantile approximatively).

So far, we have focused on the analysis of the mesoscale structure in the multilayer version of the global 
input-output network, with 44 nodes and 56 layers. In the next step, we shall compare previous results with the community structure detected from the mono-layer aggregate version, where each node is a country and a link now considers the total trade between a couple of countries. For consistency, we apply the communicability-based approach proposed in Bartesaghi et al. (2020) to the mono-layer aggregate network. We obtain seven communities (except five isolated nodes) and report their composition in Table 3.1. It is noteworthy that two big communities are detected also in this case but the composition of large communities is mainly driven by geographical patterns. This is in line with the results already detected in the literature for aggregate trade network (see Barigozzi et al. (2010), Bartesaghi et al. (2020)). It is interesting to note that the first community includes almost all European Countries. Only some well-known couples are clustered alone (see for instance Spain and Portugal in community 4 or Great Britain and Ireland in community 5). The second community groups instead together North American and Asian players.

However, the analysis of the mono-layer aggregate version may lead to an oversimplified conclusion that the input-output network among countries was simply composed by regional blocks. Indeed, we do observe the presence of large international communities in the multilayer version. The aggregate version also ignores the fact that trade inter-dependencies among countries do vary across industry-based slices. All taken together, the results allow us to emphasize how the multilayer analysis, that takes into consideration distinction among 56 industry-based layers, is able to catch more peculiar relationships between countries that cannot be detected at the aggregate level.

\begin{tabular}{ll}
\hline \hline Community & Countries \\
\hline 1 & AUT, BEL, BGR, CZE, DEU, DNK, FRA, GRC, HRV, HUN, ITA, LUX, NLD, NOR, POL, \\
& ROU, RUS, SVK, SVN, SWE, TUR \\
2 & BRA, CAN, CHN, IND, JPN, MEX, USA, ROW \\
3 & AUS, IDN \\
4 & ESP, PRT \\
5 & EST, FIN \\
6 & GBR, IRL \\
7 & LTU, LTV \\
Isolated & CHE, CYP, KOR, MLT, TWN \\
\hline \hline
\end{tabular}

Table 3.1: The composition of the communities computed on the aggregate mono-layer network in 2014.

We further investigate the internal topological properties of the communities detected in the multilayer version of the network 11 . Our main purpose here is to identify the "key actors" in these communities. To this end, we start selecting countries and sectors that belong to each identified community and we build a subnetwork. More specifically, only the links among members of the community are maintained and analyzed, while those externally built with nodes in the other communities are discarded. In particular, we focus on the two most representative communities, i.e. the Community 1 and 2, since these two largest clusters consist of different sectors from various countries. Figure 3.2 shows the ranking of the top sectors in each community based on the (total) node strengths. The results again confirm the dominance of China in the first largest community and that of USA in the second community at the industry levels as many of the influential sectors in the communities 1 and 2 are actually from these two countries. Looking at the industry codes of the most influential sectors, we also observe an interesting difference in economic and industrial structure between the two communities and hence between the two nations. While influential sectors from China are mostly

\footnotetext{
${ }^{11}$ Detailed results can be found in Figure D.1 and Table D.2 in Appendix D
} 
manufacturing-related industries, those coming from the US are more dominated by real-estate, finance, technology or service-related sectors.

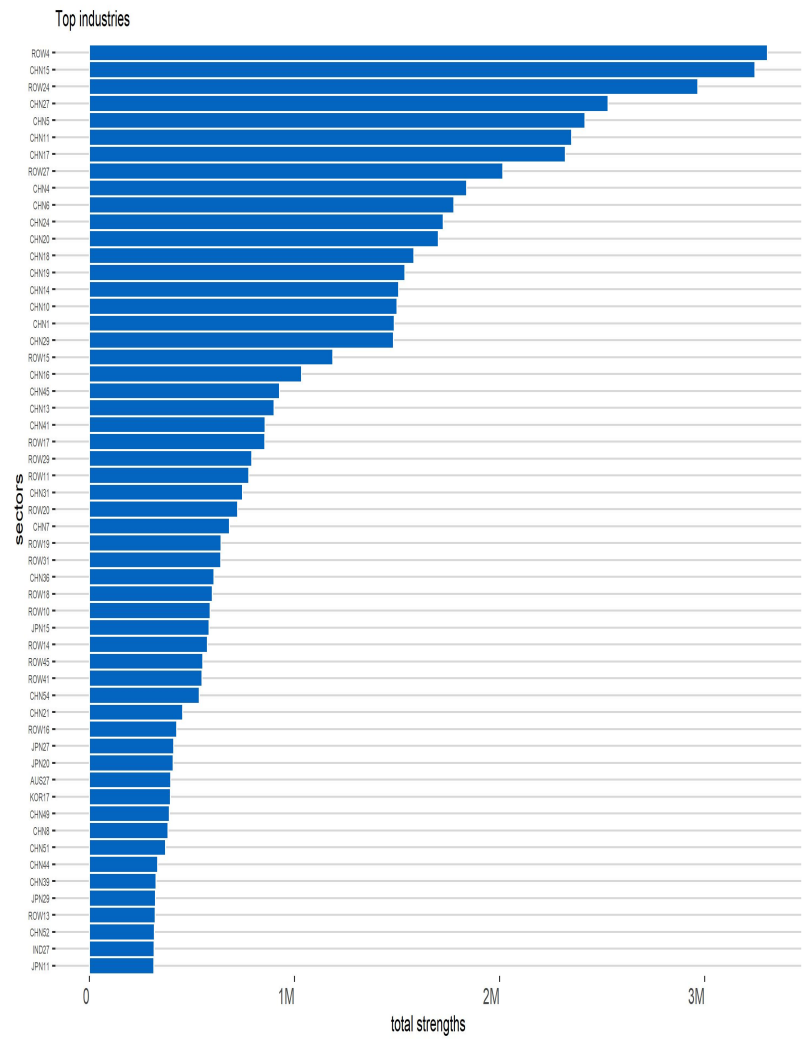

(a) Top industries in Community 1, 2014

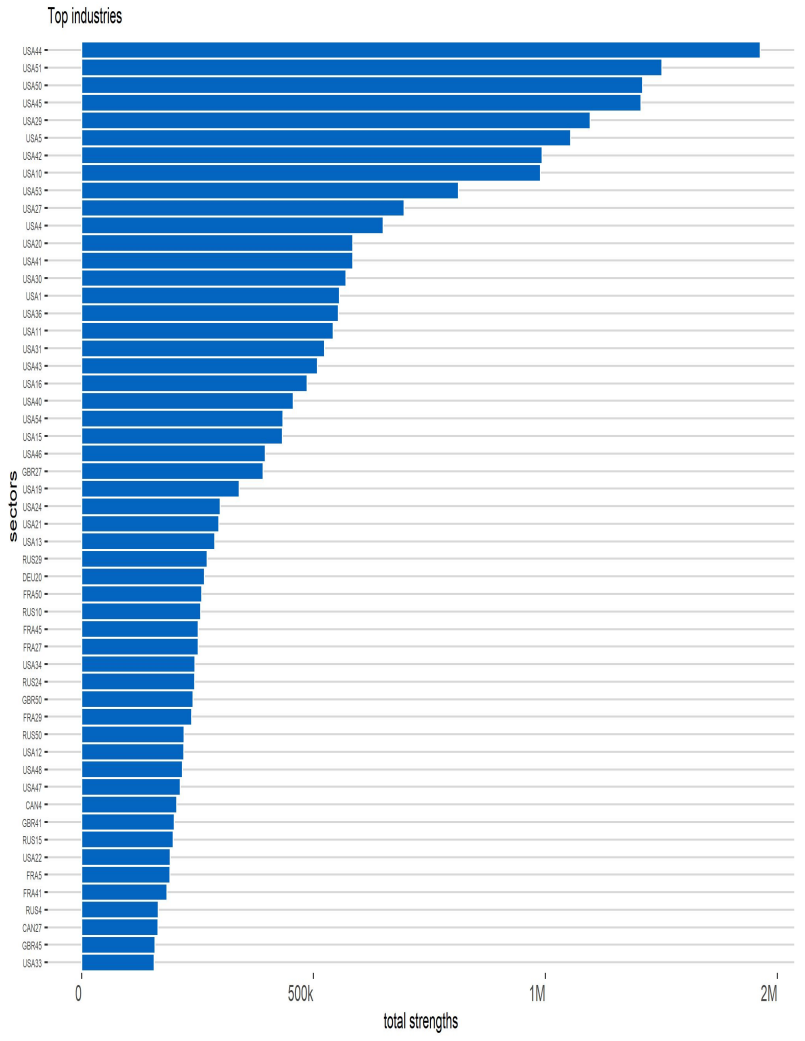

(b) Top industries in Community 2, 2014

Figure 3.2: Ranking of top industries in two important international communities in 2014, based on the (total) node strengths. Labels on the $y$ axis are given by the country code and by the number related to the sector.

\subsection{Analysis of temporal evolution of communities}

Notice that for the sake of illustration, in Section 3.2 we have focused on mesoscale structure of the considered network in the selected year 2014, which provides the latest available information we can have from the WIOD database. We shall now investigate the temporal evolution of the international communities. To this end, we considered the period 2000-2014 and we applied the method based on the multilayer communicability distance described in Section 2.3 to extract different clusters for each of these selected years. Remarkably, we find evidence that the clustering behaviors of trades in input-output among industries and countries do restructure over the period from 2000 to 2014. In general, not only the community composition changes, but the centrality rankings of the members inside each community is also reordered, with the diminishing of some industries and the emergence of new "key players".

In particular, we start analyzing the similarity between the communities obtained over time. To do this, we computed the Adjusted Rand Index (ARI) between clusters for each couple of years (see Hubert \& Arabie (1985) and Rand (1971)). This index falls in the interval [0,1] and it is equal to one only if two partitions are completely identical. Numerous measures for comparing clusterings have been proposed in the literature, 


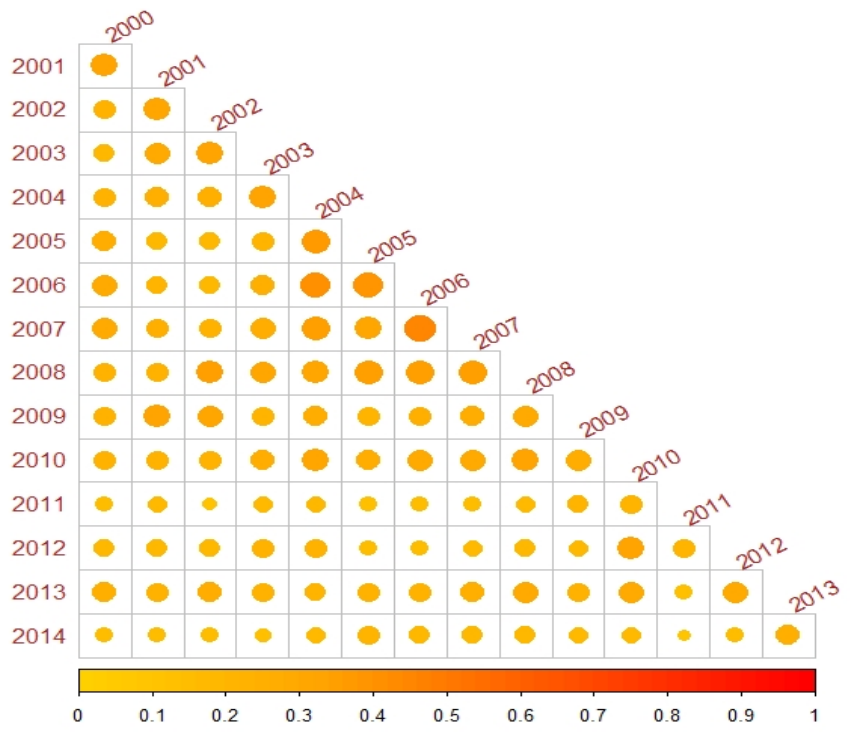

Figure 3.3: Values of ARI computed between clusters based on couple of years. The color bar and the size of bubbles indicates the value of ARI in the interval $[0,1]$. A higher value of ARI implies more similar community structures in the two corresponding years.

however the AR ${ }^{12}$ remains the most well-known and widely used (Steinley (2004)). We display in Figure 3.3 values of the index for each pair of years. We often find that while the mesoscale structure exhibits a certain level of persistence when two subsequent years are compared, it becomes less and less similar after longer time periods. Such temporal behavior can be related to substitution and margin effects from international trade theories and the basic network properties that we have analyzed in the Appendix C. Qualitative substitution and extensive margin effects, based on countries and industries that change their trade partners or even obtain new ones, are indeed not very important, since the binary version of the network in the period 2000-2014 is always (and already) very dense. Hence the network can display a certain degree of persistence. Nevertheless, similarly to what has recently been found for the World Trade network (e.g., see Felbermayr \& Kohler, 2006; Helpman et al., 2008, De Benedictis \& Tajoli, 2011), intensive margin effects play a more important role in explaining the evolution of the global input-output network. Intuitively, strengthening trade relations may take time and lead to the structural change in the whole network only once the magnitude of traded amount has been sufficiently increased. It should be also emphasized that not all trade relations are simultaneously intensified, as different sectors and countries can play different roles in such a hierarchical system. Furthermore, exogenous shocks and events such as the formation or break of trade agreements, economic zones, monetary unions may also lead to disruptive changes in the community structure of the network.

To provide deeper insights into the changes in the internal structure of the communities, we shall now

\footnotetext{
${ }^{12}$ Given a set of elements and two different partitions, we compute the sum of the number of pairs of elements that are in the same subset in both partitions and the number of pairs of elements that are in the different subsets in both partitions. The ratio between this value and the total number of pairs is the Rand Index and gives a frequency of occurrence of agreements over the total pairs. ARI is the corrected-for-chance version of the Rand index and it gives the overall concordance of two methods taking into account that the agreement between partitions could arise by chance alone.
} 
analyze their composition over time 13 As shown in Figure 3.4 (panel a) starting in 2000, the first largest community, with 571 members, is mainly composed by sectors from the US, Japan and interestingly several ones from China. In contrast, the second largest cluster (with 167 members) mainly consists of industries from the former Eastern Bloc's countries, led by those from Russia and Poland 14

Over the next few consecutive years, first major changes are observed in 2002 (see Figure 3.4 (panel b)). We have indeed an important reduction of the total number of communities and a higher concentration in the largest group with 895 members. In particular, Community 1 is enriched by the inclusion of several sectors from European countries: Austria, Belgium, Germany and Great Britain join the other European countries (Italy, France, Spain, Ireland) in this big community. On the other hand, the presence of Eastern Bloc's country in Community 2 is reducing. The composition of Community 2 is indeed affected by the effects of the disintegration of the Eastern Bloc with the dissolution of the Warsaw Pact and Comecon. We find that the former members of the Warsaw Pact, that joined NATO (as Poland, Hungary and Czech Republic) in 1999, moved from Community 2 (including Russia) to Community 1 (including United States) ${ }^{15}$. A relevant aspect that could lead to major changes in the mesoscale structure of the global input-output network from 2002 is the entrance of China in World Trade Organization (WTO) in December 2001 16 China's accession to the WTO brought a number of benefits to both China as well as the world. Reduced barriers to trade and larger foreign direct investment inflows boosted export as well as import markets of this country.

Notice that the composition of the first largest community remains almost the same in the next few consecutive years (as can be seen from Figure 3.4). However, some Chinese sectors become more and more important. For example, the role of Chinese manufacturing industries assumes in 2004 a higher relevance in this community (see also Figure D.4 (a) in the Appendix D).

This result is in line with Chen (2009) and De Benedictis \& Tajoli (2011) that show an important increase of labor-intensive manufactured goods' exportation and of importation of raw materials that come from China and the other developing Asian economies (aggregated in ROW) during that period. In contrast, looking at the internal structure of the second largest community in 2004, we find that sectors from the former Eastern Bloc's countries play a less important role, but those from Belgium and France become more dominant (for details see also Figure D.4 in the Appendix D.

Overall, we observe that the structure of the largest international communities still continues to evolve in the subsequent years. For example, as for the data in 2009, a number of influential industries from China no longer stay in the first largest community with those from US but emerge as the "key players" in the second largest one. In particular, as displayed in Figure 3.4 (d), a large community dominated by Asian countries is noticeable (in particular formed by sectors of South Korea, China and Taiwan) 17 The role of Asian countries still continue to increase over time, leading China in 2014 (as already reported and discussed in Section 3.2 to form the largest international community together with some sectors from the other Asian countries such as South Korea, Taiwan, India, Japan. Altogether, the aforementioned observations may be

\footnotetext{
${ }^{13}$ For the sake of illustration and conciseness, we only report the results related to some selected years before 2014 . Results for other years are available upon request to the authors.

${ }^{14}$ See also Figure D.2 in the Appendix D for additional results.

${ }^{15}$ See also Figure $\overline{D .3}$ in the Appendix D for additional results.

${ }^{16}$ See, for example, Subramanian \& Wei (2007) for further discussions on the impacts of WTO on trade.

${ }^{17}$ Additional results can be found in Figure D.5 Appendix D
} 
the signals that the global trade in input-output system has been moving from a former US-Russia bipolar system to another bipolar (or multipolar) one, with the rising role of sectors from China in the recent years.

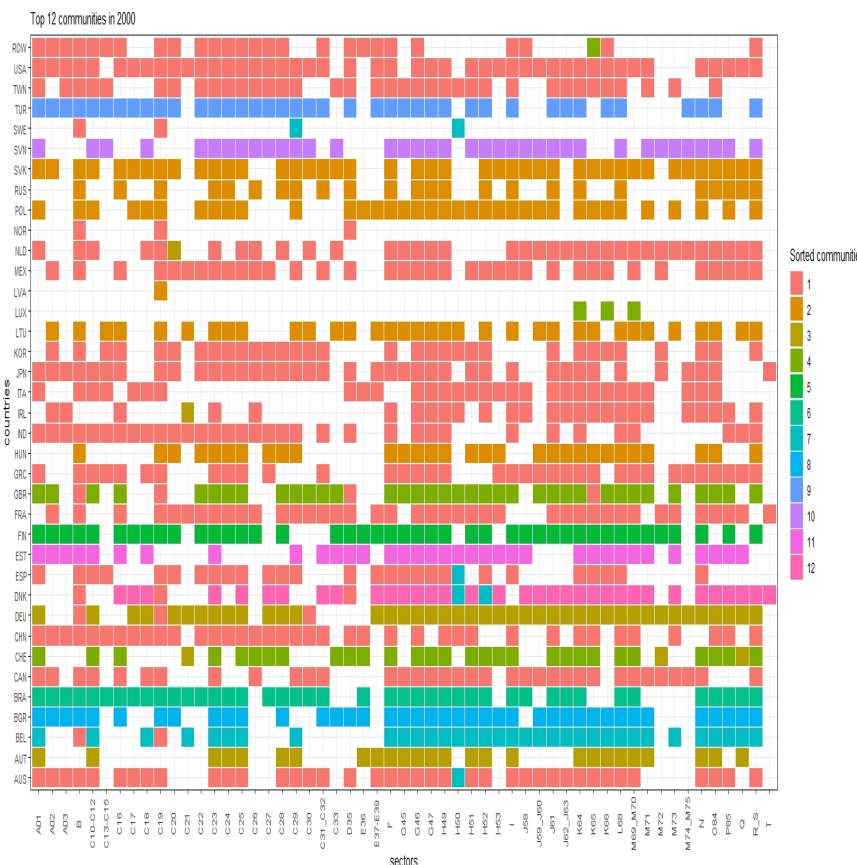

(a) Top communities in 2000

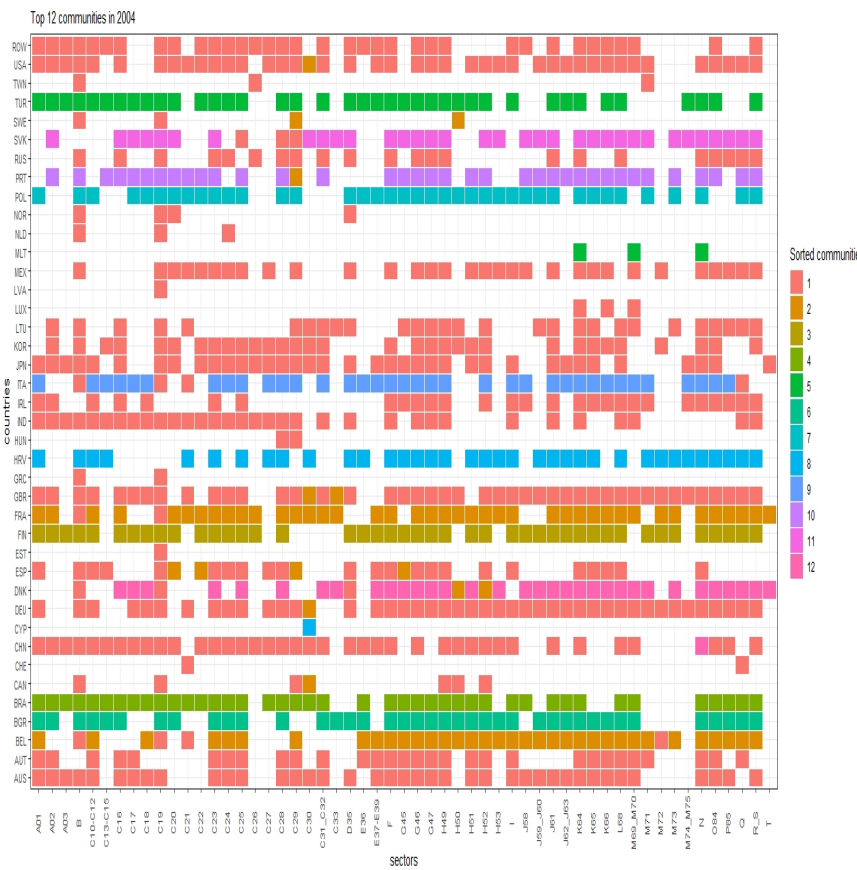

(c) Top communities in 2004

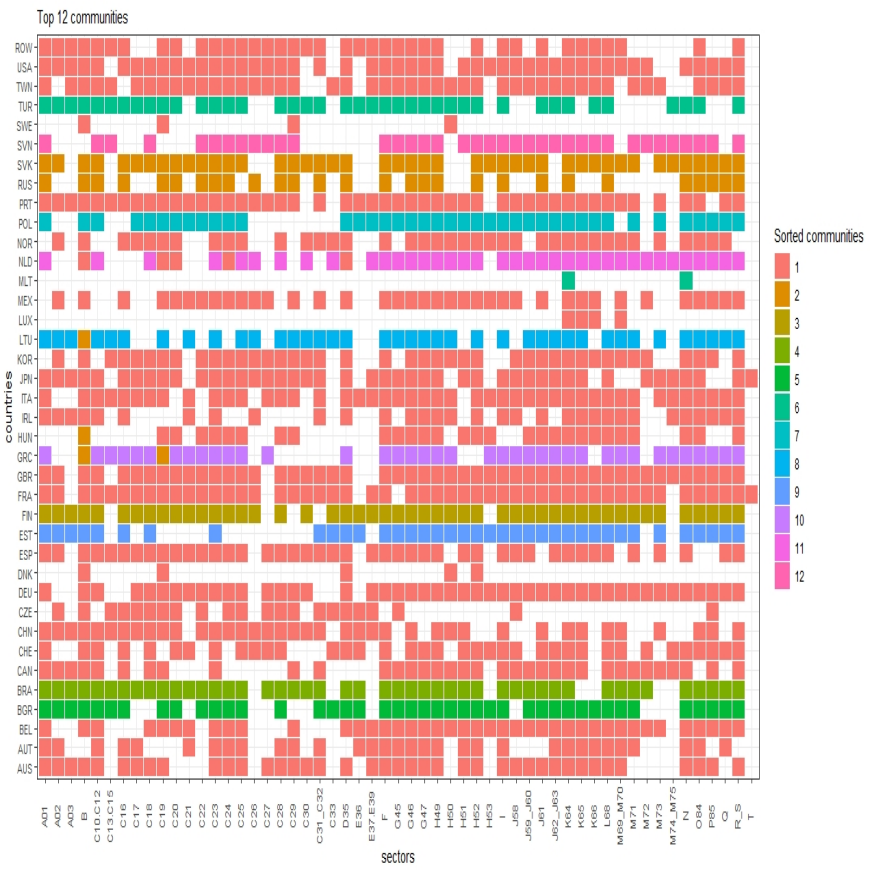

(b) Top communities in 2002

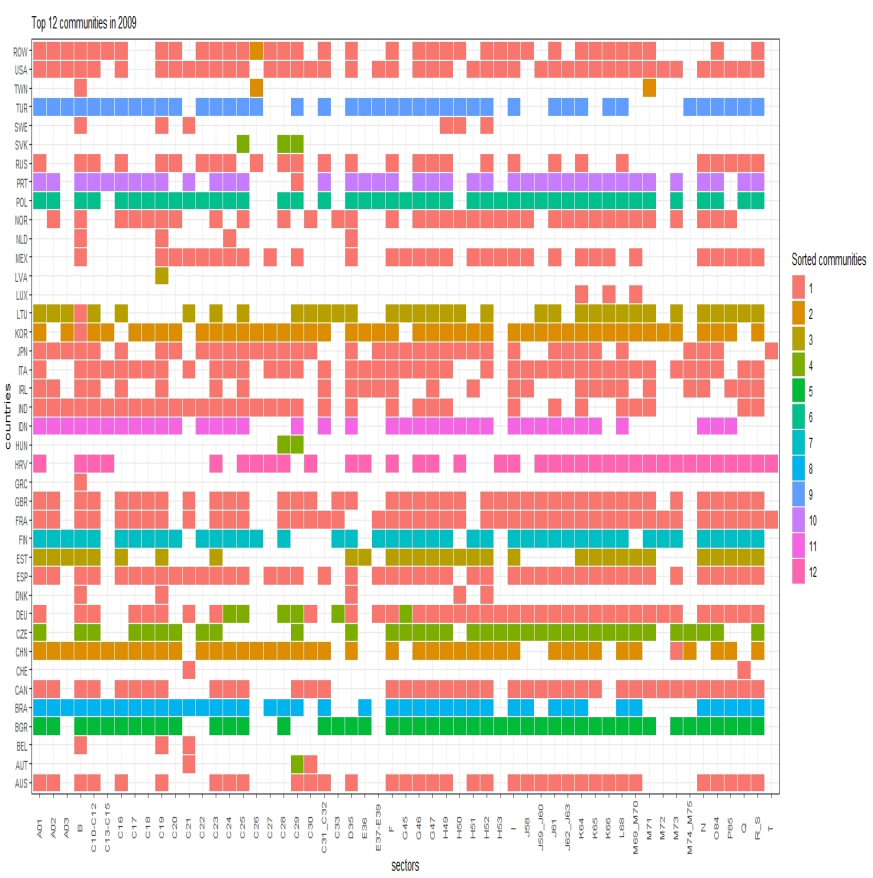

(d) Top communities in 2009

Figure 3.4: Detected communities in the global trade network in input-output in selected years 2000, 2002, 2004 and 2009. 
To bring our temporal analysis of the mesoscale structure of the global trade network in input-output to a close, we report in Figure 3.5 the Gini index, computed for each year, at either country or sector level ${ }^{18}$

We notice, on average, a lower level of heterogeneity for countries, confirming the presence of relevant domestic trades (see Figure 3.5, panel (a)). As reported and discussed in Section 3.2 industries from a number of countries engage less actively in the global input-output network, and many of them still mainly rely on their domestic counterparts. The Gini indices for these countries, therefore, are relatively lower. On the other hand, a higher level of heterogeneity is observed for several Eastern European and Mediterranean Sea countries. We argue that for these countries, a relatively stronger international diversification occurs primarily at the (quantitative) intensive margin effects rather than the extensive ones, since the binary version of the network capturing the existence of trade links is already very dense and does not change very much. Indeed, these countries derived great benefits from their proximity to the European Union, large amounts of investment from their Western European neighbors and the far-ranging domestic reforms on which their accession was conditional (see for details Ait Ali et al. (2022)). In a similar vein, some Asian developing countries benefited from a strong export orientation and the increased intra-regional integration, also due to the proximity to the Chinese growth pole. Relevant players (as United States, Germany, Japan and China) show instead a moderate level of heterogeneity due to a greater balance between domestic and international trade linkages.

Concerning sectors (see Figure 3.5 panel (b)), we notice relatively higher values for their Gini indices, with few exceptions. As already observed for 2014 in Section 3.2 the two sectors "Mining and quarrying (B)" and "Manufacture of coke and refined petroleum products (C19)" also show systematically a lower heterogeneity and are mainly involved in Community 1 in the previous years. This is not very surprising since from a perspective of the factors of production, over different countries worldwide, these two industries are very closely connected to each other.

In addition, major changes in the mesoscale structure of the global input-output network are again confirmed by a significant drop in the Gini index in 2002 for all sectors, where a very high concentration of countries and sectors in the largest community is observed. As discussed before, the restructure of trade relations among European countries, the disintegration of the Eastern Bloc, the rising role of sectors from China after its accession to WTO, etc. may explain such noticeable changes in the network in that year.

${ }^{18}$ Notice that we have computed the Gini index for each country as well as for each sector in 2014 (Tables D.1 and D.2 in Appendix D last column). Then we compute the index for each country and sector in the other years, to examine its evolution over time from 2000 to 2014. As explained previously, a higher value of this index for a country means that on average its sectors are more spread out between different communities. In contrast, a higher value of this index for a sector (layer) implies that, in the same traded layer, countries are on average split in various communities. 


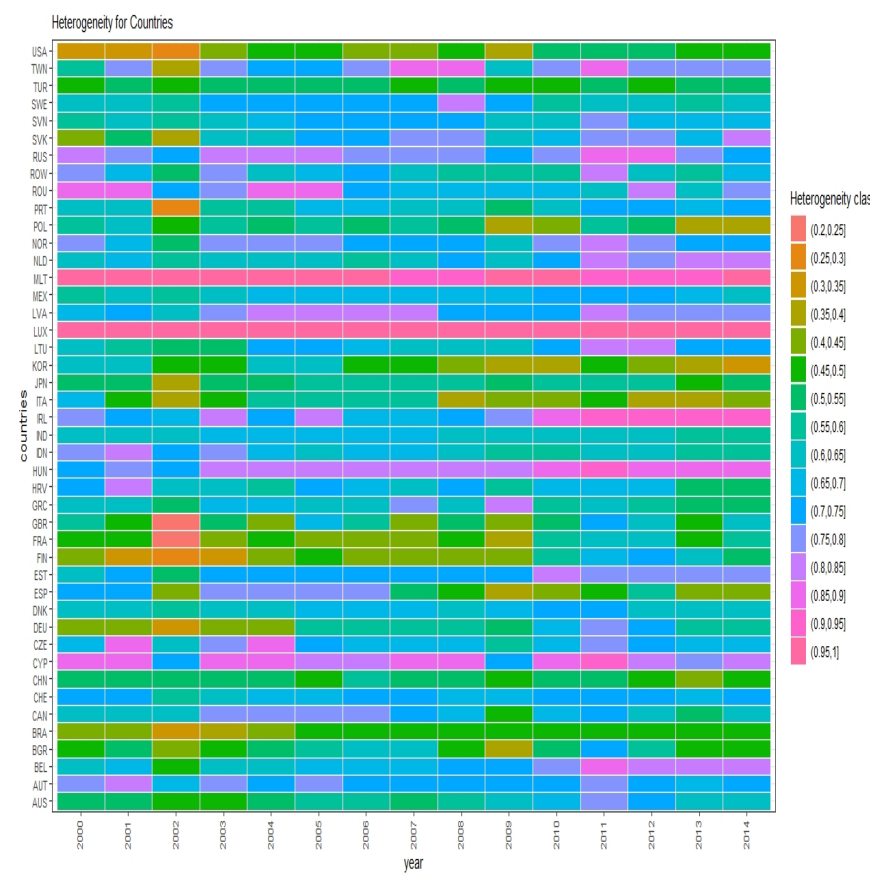

(a) Heterogeneity index computed for each country

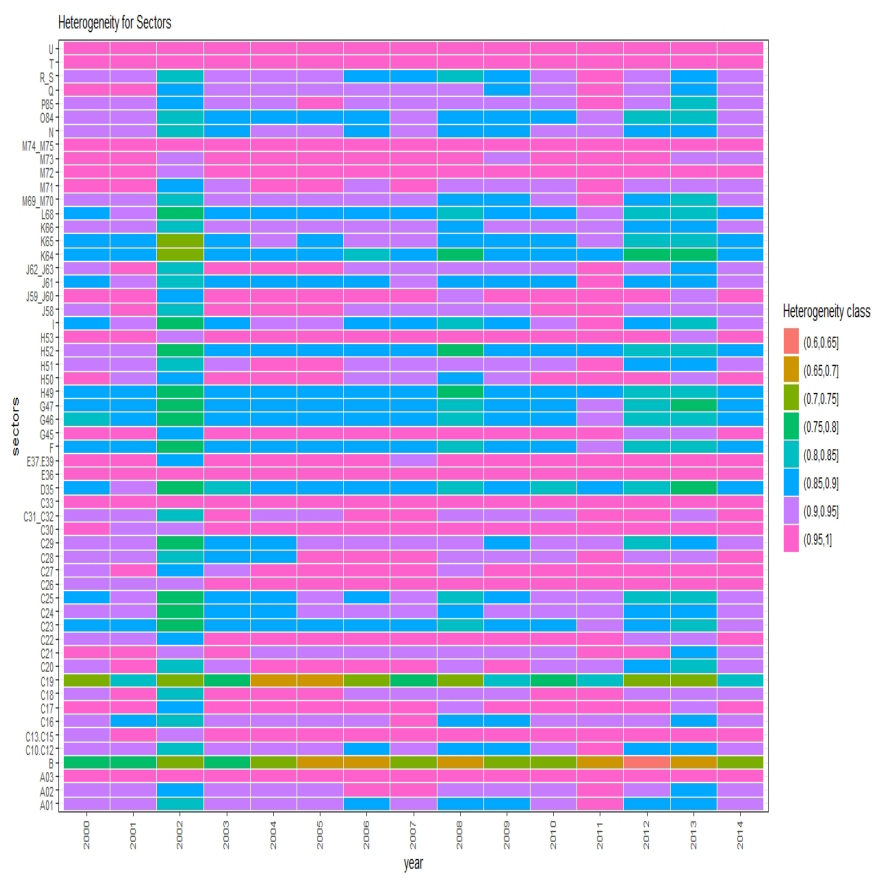

(b) Heterogeneity index computed for each sector

Figure 3.5: Heterogeneity index computed for each year at country and sector level, respectively. The color bar indicates the value of Gini index in the interval $[0,1]$. The index provides an indication of the dispersion of sectors (countries) for each country (sector) along the communities. A larger value of this index implies a higher degree of dispersion.

\section{Conclusions}

This work analyses the global trade network in input-output, using the recently released WIOD database. We show that, by viewing the network through the lens of a multilayer architecture, we are able to extract richer information on interdependencies between countries and industries around the world that cannot be easily detected at the aggregate mono-layer level.

We first analyse the heterogeneity and the diversification in input-output relationships and find that although the list of trading partners is generally broad, some sectors trade more intensively with a number of other sectors in different countries. We then view countries as nodes and industries as layers and explore the similarities and interactions among the layers. Again, in the weighted version, we observe that the similarity levels and the interaction strengths are varied across pairs of layers and that few couples of layers tend to be more overlapped and/or more strongly interacting with each other. Such first insights motivate us to examine the multilayer architecture at a broader scale rather the node-node or layer-layer interrelations alone.

As investigated in our work, at the mesoscale level there exist several large international communities in which some countries trade their inputs and outputs more intensively in some specific industry-based layers. In such a network structure, the world somewhat seems to have bipolar or multipolar trade system with different clusters that are more internally cohesive.

Furthermore, interestingly, we also observe that the clustering behaviors of trades in input-output among industries and countries restructure over the period from 2000 to 2014. In general, not only the internal 
composition changes, but the centrality rankings of the members inside each community also reorder, with the diminishing role of industries from some countries and the growing importance of those from some other countries.

As in 2000, the first largest community is still mainly comprised by sectors from the US, Japan together with several sectors from China. In contrast, the second largest cluster consists of industries mostly from the former Eastern Bloc's countries (e.g. Russia and Poland). As in the data for 2014, the most recent year available in the WIOD database, we identify an Australian-Asian community, where relevant industries of main Asian players (China, India, Japan, South Korea) and Australia are clustered together. On the other hand, those from countries involved in the North American Free Trade Agreement (Canada, Mexico and USA) belongs to the next largest community.

The empirical results obtained from our present work provide several meaningful implications. First, it is worth mentioning that large international clusters comprised by different industries from different nations detected in our present work may pave the potential pathways for large cascading failures. In particular, a shock to a "key" sector (hub) of a "key" country in one of these clusters could be transmitted and propagated to many other foreign sectors located in the same cluster via cross-border input-output relations, and possibly lead to a large downturn in the global trade. As shown during the time of the Covid pandemics, the shortages of inputs provided by some industries can lead to disruptions in a number of subsequent dependent industries in other countries (e.g., Eppinger et al., 2020). In addition, the presence of such large international clusters, in which members of each cluster interact more strongly among themselves, may also imply meaningful network origins (i.e., those related to international input-output relations) of business cycle synchronizations among different countries in the world (e.g., Burstein et al. 2008, Johnson, 2014). Moreover, as pointed out in our present work, the multilayer analysis helps to uncover the complexity of the relations among countries when they trade within and between different layers-based industrial sectors. The proposed approach also allows us to explore the interdependencies among layers rather than just the node-node pairwise relations in the aggregate mono-layer analysis. As shown in Lee \& Goh (2016), Korniyenko et al. (2018) and in Tzavellas (2022), more severe cascading effects can occur in an architecture of multiple layers. This is mainly because the simultaneous presence of intralayer as well as interlayer linkages in such an architecture can lead to collective dynamics of interdependent layers and create multiple potential channels of the diffusion of shocks among countries that are ignored by the analysis of the aggregate version or the analysis of single layers alone.

Our present work opens several directions for future research. First, the findings on the emergence of several international communities and their structural changes over time need to be investigated further to determine the underlying economic and other potential mechanisms. Second, since in this study we focus on the mesoscale structure and the related properties of the global input-output network, in our future work, we plan to extend our analysis to study and quantify the other important network measures and properties such as the multilayer clustering coefficients and the multilayer centrality (e.g. see De Domenico et al., 2013. Bartesaghi et al. 2022). Third, we believe that incorporating additional layers representing other economic relations such as financial links, trade in final goods and services on top of the input-output interrelations among countries would be able to give a more comprehensive and richer analysis of the multilayer architecture of the world-wide economic network. Such an analysis certainly provides useful inputs to the study of different potential channels of cascading failures and transmission of shocks in the global economy (e.g., see Lee \& Goh, 2016: Korniyenko et al. 2018. 


\section{References}

Acemoglu, D., Akcigit, U., \& Kerr, W. (2016). Networks and the macroeconomy: An empirical exploration. NBER Macroeconomics Annual, 30, 273-335.

Acemoglu, D., Carvalho, V. M., Ozdaglar, A., \& Tahbaz-Salehi, A. (2012). The network origins of aggregate fluctuations. Econometrica, 80, 1977-2016.

Acemoglu, D., Ozdaglar, A., \& Tahbaz-Salehi, A. (2017). Microeconomic origins of macroeconomic tail risks. American Economic Review, 107, 54-108.

Ait Ali, A., Doghan, M., Bhatti, B., Braga, C., Dadush, U., Darandary, A., González, A., \& Poitiers, N. (2022). Diversification and the World Trading System. T-20 Secretariat Secretariat T-20.

Anderson, J. E. (1979). A theoretical foundation for the gravity equation. The American Economic Review, $69,106-116$.

Antonietti, R., Falbo, P., Fontini, F., Grassi, R., \& Rizzini, G. (2022). The world trade network: country centrality and the covid-19 pandemic. Applied Network Science, 7, 1-29.

Antràs, P., \& de Gortari, A. (2020). On the geography of global value chains. Econometrica, 84, 1553-1598.

Atalay, E. (2017). How important are sectoral shocks? American Economic Journal: Macroeconomics, 9, $254-80$.

Baldwin, R., \& Lopez-Gonzalez, J. (2015). Supply-chain trade: A portrait of global patterns and several testable hypotheses. The World Economy, 38, 1682-1721.

Baldwin, R. E. (2006). Multilateralising regionalism: Spaghetti bowls as building blocs on the path to global free trade. The World Economy, 29, 1451-1518.

Bargigli, L., di Iasio, G., Infante, L., Lillo, F., \& Pierobon, F. (2015). The multiplex structure of interbank networks. Quantitative Finance, 15, 673-691.

Barigozzi, M., Fagiolo, G., \& Garlaschelli, D. (2010). Multinetwork of international trade: A commodityspecific analysis. Phys. Rev. E, 81, 046104.

Bartesaghi, P., Clemente, G. P., \& Grassi, R. (2020). Community structure in the world trade network based on communicability distances. Journal of Economic Interaction and Coordination, (pp. 1-37).

Bartesaghi, P., Clemente, G. P., \& Grassi, R. (2022). A tensor-based unified approach for clustering coefficients in financial multiplex networks. Information Sciences, 601, 268-286.

Battiston, S., Caldarelli, G., \& Garas, A. (2018). Multiplex and multilevel networks.

Bianconi, G. (2018). Multilayer networks: Structure and function. Oxford University Press.

Boehm, C. E., Flaaen, A., \& Pandalai-Nayar, N. (2019). Input Linkages and the Transmission of Shocks: Firm-Level Evidence from the 2011 Tōhoku Earthquake. The Review of Economics and Statistics, 101, 60-75. 
Bostandzic, D., \& Weiß, G. N. (2018). Why do some banks contribute more to global systemic risk? Journal of Financial Intermediation, 35, $17-40$.

Burstein, A., Kurz, C., \& Tesar, L. (2008). Trade, production sharing, and the international transmission of business cycles. Journal of Monetary Economics, 55, 775-795.

Carvalho, V. M. (2014). From micro to macro via production networks. Journal of Economic Perspectives, 28, 23-48.

Carvalho, V. M., Nirei, M., Saito, Y., \& Tahbaz-Salehi, A. (2016). Supply Chain Disruptions: Evidence from the Great East Japan Earthquake. Working Paper No. 2017-01 Becker Friedman Institute for Research in Economics.

Cerina, F., Zhu, Z., Chessa, A., \& Riccaboni, M. (2015). World input-output network. PLOS ONE, 10, $1-21$.

Cetorelli, N., \& Goldberg, L. (2011). Global banks and international shock transmission: Evidence from the crisis. IMF Economic Review, 59, 41-76.

Chang, C., Liao, W., Chen, Y., \& Liou, L. (2016). A mathematical theory for clustering in metric spaces. IEEE Transactions on Network Science and Engineering, 3, 2-16.

Chen, C. (2009). China's Integration with the Global Economy: WTO Accession, Foreign Direct Investment, and International Trade. Cheltenham, UK: Edward Elgar Pub.

Ciccone, A. (2002). Input chains and industrialization. The Review of Economic Studies, 69, 565-587.

Crofts, J., \& Higham, D. (2009). A weighted communicability measure applied to complex brain networks. Journal of the Royal Society, Interface / the Royal Society, 6, 411-4.

De Benedictis, L., \& Tajoli, L. (2011). The world trade network. The World Economy, 34, 1417-1454.

De Domenico, M., Solé-Ribalta, A., Cozzo, E., Kivelä, M., Moreno, Y., Porter, M. A., Gómez, S., \& Arenas, A. (2013). Mathematical formulation of multilayer networks. Phys. Rev. X, 3, 041022.

Di Giovanni, J., \& Levchenko, A. A. (2010). Putting the parts together: Trade, vertical linkages, and business cycle comovement. American Economic Journal: Macroeconomics, 2, 95-124.

Di Giovanni, J., Levchenko, A. A., \& Mejean, I. (2018). The micro origins of international business-cycle comovement. American Economic Review, 108, 82-108.

Dietzenbacher, E., Romero Luna, I., \& Bosma, N. S. (2005). Using Average Propagation Lengths to Identify Production Chains in the Andalusian Economy/Empleando Longitudes Medias de Propagación para identificar Cadenas Productivas en la Economía Andaluza. Estudios de Economia Aplicada, 23, 405-422.

Eppinger, P., Felbermayr, G. J., Krebs, O., \& Kukharskyy, B. (2020). Covid-19 Shocking Global Value Chains. CESifo Working Paper Series 8572 CESifo.

Estrada, E. (2011). The Structure of Complex Networks: Theory and Applications. Oxford University Press. 
Estrada, E., \& Hatano, N. (2008). Communicability in complex networks. Phys. Rev. E, 77, 036111.

Estrada, E., Hatano, N., \& Benzi, M. (2012). The physics of communicability in complex networks. Physics Reports, 514, $89-119$.

Fagiolo, G., Reyes, J., \& Schiavo, S. (2008). On the topological properties of the world trade web: A weighted network analysis. Physica A: Statistical Mechanics and its Applications, 387, 3868-3873. Applications of Physics in Financial Analysis.

Felbermayr, G. J., \& Kohler, W. (2006). Exploring the intensive and extensive margins of world trade. Review of World Economics / Weltwirtschaftliches Archiv, 142, 642-674.

Fortunato, S. (2010). Community detection in graphs. Physics Reports, 486, $75-174$.

Garlaschelli, D., \& Loffredo, M. I. (2005). Structure and evolution of the world trade network. Physica A: Statistical Mechanics and its Applications, 355, 138-144. Market Dynamics and Quantitative Economics.

Gemmetto, V., \& Garlaschelli, D. (2014). Multiplexity versus correlation: The role of local constraints in real multiplexes. Scientific Reports, 5.

Gemmetto, V., Squartini, T., Picciolo, F., Ruzzenenti, F., \& Garlaschelli, D. (2016). Multiplexity and multireciprocity in directed multiplexes. Phys. Rev. E, 94, 042316.

Giammetti, R., Russo, A., \& Gallegati, M. (2020). Key sectors in input-output production networks: An application to brexit. The World Economy, 43, 840-870.

Hale, G., Kapan, T., \& Minoiu, C. (2019). Shock Transmission Through Cross-Border Bank Lending: Credit and Real Effects. The Review of Financial Studies, .

Helpman, E., Melitz, M., \& Rubinstein, Y. (2008). Estimating Trade Flows: Trading Partners and Trading Volumes*. The Quarterly Journal of Economics, 123, 441-487.

Hirschman, A. O. (1964). The paternity of an index. The American Economic Review, 54, 761-762.

Hubert, L., \& Arabie, P. (1985). Comparing partitions. Journal of Classification, 2, 193-218.

Johnson, R. C. (2014). Trade in intermediate inputs and business cycle comovement. American Economic Journal: Macroeconomics, 6, 39-83. doi $10.1257 /$ mac.6.4.39.

Kleimeier, S., Sander, H., \& Heuchemer, S. (2013). Financial crises and cross-border banking: New evidence. Journal of International Money and Finance, 32, 884-915.

Korniyenko, Y., Patnam, M., Del Río-Chanona, R. M., \& Porter, M. (2018). Evolution of the global financial network and contagion: A new approach. IMF Staff Papers, 18.

Kvålseth, T. O. (2018). Relationship between concentration ratio and herfindahl-hirschman index: A reexamination based on majorization theory. Heliyon, 4, e00846.

Lee, K.-M., \& Goh, K. I. (2016). Strength of weak layers in cascading failures on multiplex networks: case of the international trade network. Scientific Reports, (pp. 1-9). 
Leon, C., Berndsen, R., \& Renneboog, L. (2014). Financial stability and interacting networks of financial institutions and market infrastructures. SSRN Electronic Journal, .

Liu, E. (2019). Industrial Policies in Production Networks*. The Quarterly Journal of Economics, 134, $1883-1948$.

Long, J. B., \& Plosser, C. I. (1983). Real business cycles. Journal of Political Economy, 91, 39-69.

Luu, D. T., \& Lux, T. (2019). Multilayer overlaps and correlations in the bank-firm credit network of spain. Quantitative Finance, 19, 1953-1974.

Luu, D. T., Lux, T., \& Yanovski, B. (2017). Structural correlations in the italian overnight money market: An analysis based on network configuration models. Entropy, 19.

Luu, D. T., Napoletano, M., Fagiolo, G., Roventini, A., \& Sgrignoli, P. (2018a). Shock Diffusion in the European Production Network:Systemic Importance and Cascading Failures. ISIGrowth Working Paper 38/2018 July ISIGrowth.

Luu, D. T., Napoletano, M., Fagiolo, G., Roventini, A., \& Sgrignoli, P. (2018b). Uncovering the Network Complexity in Input-Output Linkages among Sectors in European Countries. ISIGrowth Working Paper 17/2018 May ISIGrowth.

Miller, R. E., \& Blair, P. D. (2009). Input-Output Analysis: Foundations and Extensions. Cambridge University Press.

Mundt, P. (2021). The formation of input-output architecture: Evidence from the european union. Journal of Economic Behavior \& Organization, 183, 89-104.

Newman, M. E. J. (2003). The structure and function of complex networks. SIAM Rev., 45, 167-256.

Pack, H., \& Saggi, K. (2006). The case for industrial policy: a critical survey. Policy Research Working Paper Series 3839 The World Bank.

Park, C.-Y., \& Shin, K. (2020). Contagion through national and regional exposures to foreign banks during the global financial crisis. Journal of Financial Stability, 46, 100721.

Poledna, S., Molina-Borboa, J. L., Martínez-Jaramillo, S., [van der Leij], M., \& Thurner, S. (2015). The multi-layer network nature of systemic risk and its implications for the costs of financial crises. Journal of Financial Stability, 20, $70-81$.

Rand, W. M. (1971). Objective criteria for the evaluation of clustering methods. Journal of the American Statistical Association, 66, 846-850.

Russo, M., Alboni, F., Sanginés, J., De Domenico, M., Mangioni, G., Righi, S., Righi, S., \& Simonazzi, A. (2022). The Changing Shape of the World Automobile Industry: A Multilayer Network Analysis of International Trade in Components and Parts. Working Paper Series 173 Institute for New Economic Thinking. 
Serrano, M., Boguñá, M., \& Vespignani, A. (2007). Patterns of dominant flows in the world trade web. Journal of Economic Interaction and Coordination, 2, 111-124.

Shin, H. S. (2012). Global banking glut and loan risk premium. IMF Economic Review, 60, 155-192.

Silva, T. C., da Silva, M. A., \& Tabak, B. M. (2017). Systemic risk in financial systems: A feedback approach. Journal of Economic Behavior \& Organization, 144, 97-120.

Steinley, D. (2004). Properties of the Hubert-Arabie adjusted Rand index. Psychol Methods, 9, 386-396.

Subramanian, A., \& Wei, S.-J. (2007). The wto promotes trade, strongly but unevenly. Journal of International Economics, 72, 151-175.

Timmer, M., Los, B., Stehrer, R., \& de Vries, G. (2016). An Anatomy of the Global Trade Slowdown based on the WIOD 2016 Release. GGDC Research Memorandum GD-162 Groningen Growth and Development Centre, University of Groningen.

Timmer, M. P., Dietzenbacher, E., Los, B., Stehrer, R., \& de Vries, G. J. (2015). An illustrated user guide to the world input-output database: the case of global automotive production. Review of International Economics, 23, 575-605.

Tzavellas, H. (2022). A Multilayer View of Systemic Importance and Aggregate Fluctuations. Working Paper Virginia Tech. 


\section{Appendix A. Node centrality in a multilayer architecture}

We report here the definitions of node centrality measures applied in the text. The intralayer in-strength and in-degree for each node $i$ in a layer $\alpha$ are given by

$$
s_{i, i n}^{[\alpha \leftarrow \alpha]}=\sum_{j \neq i} W_{j i}^{[\alpha, \alpha]}
$$

and

$$
k_{i, i n}^{[\alpha \leftarrow \alpha]}=\sum_{j \neq i} A_{j i}^{[\alpha, \alpha]} .
$$

In a similar vein, we can define the intralayer out-strength and out-degree of node $i$ in layer $\alpha$ as

$$
\begin{aligned}
& s_{i, \text { out }}^{[\alpha \rightarrow \alpha]}=\sum_{j \neq i} W_{i j}^{[\alpha, \alpha]}, \\
& k_{i, \text { out }}^{[\alpha \rightarrow \alpha]}=\sum_{j \neq i} A_{i j}^{[\alpha, \alpha]} .
\end{aligned}
$$

In addition, for every pair of different layers $\alpha$ and $\beta$, one can quantify the interlayer in-degree and in-strength of interactions from all nodes in a layer $\beta$ to a specific node $i$ in layer $\alpha$ as

$$
\begin{aligned}
& s_{i, i n}^{[\alpha \leftarrow \beta]}=\sum_{j \neq i} W_{j i}^{[\alpha, \beta]}, \\
& k_{i, i n}^{[\alpha \leftarrow \beta]}=\sum_{j \neq i} A_{j i}^{[\alpha, \beta]} .
\end{aligned}
$$

Similarly, the interlayer out-strength and out-degree from a particular node $i$ in the layer $\alpha$ to all nodes in a layer $\beta$ are given by

$$
s_{i, o u t}^{[\alpha \rightarrow \beta]}=\sum_{j \neq i} W_{i j}^{[\alpha, \beta]}
$$

and

$$
k_{i, \text { out }}^{[\alpha \rightarrow \beta]}=\sum_{j \neq i} A_{i j}^{[\alpha, \beta]} .
$$

In the context of an input-output network, the aforementioned degrees and strengths represent the distributions of the numbers of trading partners and of the magnitudes of trade in input-output across nodes, respectively. A higher incoming (outgoing) degree of a node indicates that it has a more diversified portfolio of input sellers (output buyers) in a particular layer. Nodes with higher incoming (outgoing) strengths trade their inputs (outputs) more intensively with the rest of nodes in a certain layer.

The total in-strength and in-degree of a node $i$ in a layer $\alpha$ from nodes in all $L$ layers can be straightforwardly extended as

$$
\begin{aligned}
& s_{i, \text { in }}^{[\alpha, \text { total }]}=\sum_{\beta} s_{i, i n}^{[\alpha \leftarrow \beta]}, \\
& k_{i, \text { in }}^{[\alpha, \text { total }]}=\sum_{\beta} k_{i, i n}^{[\alpha \leftarrow \beta]} .
\end{aligned}
$$


Analogously, we can define the total out-strength and out-degree of a node $i$ in a layer $\alpha$ across all layers as

$$
\begin{aligned}
& s_{i, \text { out }}^{[\alpha, \text { total }]}=\sum_{\beta} s_{i, \text { out }}^{[\alpha \rightarrow \beta]}, \\
& k_{i, \text { out }}^{[\alpha, \text { total }]}=\sum_{\beta} k_{i, \text { out }}^{[\alpha \rightarrow \beta]} .
\end{aligned}
$$

Notice that from the definitions of the total in-strength $s_{i, \text { in }}^{[\alpha, t a t]}$ and the intralayer in-strength $s_{i, i n}^{[\alpha \leftarrow \alpha]}$, we can easily obtain the total interlayer in-strength, which is given by

$$
s_{i, i n}^{[\alpha, \text { inter }]}=s_{i, \text { in }}^{[\alpha, \text { total }]}-s_{i, i n}^{[\alpha \leftarrow \alpha]}=\sum_{\beta \neq \alpha} s_{i, i n}^{[\alpha \leftarrow \beta]}
$$

This captures the overall intensity of the dependency of node $i$ in the layer $\alpha$ on the inputs provided by nodes from the other layers. Similarly, one can derive the total interlayer in-degree, the total interlayer out-strength and the total interlayer out-degree:

$$
\begin{aligned}
& k_{i, \text { in }}^{[\alpha, \text { inter }]}=k_{i, \text { in }}^{[\alpha, \text { total }]}-k_{i, \text { in }}^{[\alpha \leftarrow \alpha]}=\sum_{\beta \neq \alpha} k_{i, \text { in }}^{[\alpha \leftarrow \beta]}, \\
& s_{i, \text { out }}^{[\alpha, \text { inter }]}=s_{i, \text { out }}^{[\alpha, \text { total }]}-s_{i, \text { out }}^{[\alpha \rightarrow \alpha]}=\sum_{\beta \neq \alpha} s_{i, \text { out }}^{[\alpha \rightarrow \beta]},
\end{aligned}
$$

and

$$
k_{i, \text { out }}^{[\alpha, \text { inter }]}=k_{i, \text { out }}^{[\alpha, \text { total }]}-k_{i, \text { out }}^{[\alpha \rightarrow \alpha]}=\sum_{\beta \neq \alpha} k_{i, \text { out }}^{[\alpha \rightarrow \beta]} .
$$




\section{Appendix B. Lists and tables}

In this subsection, we summarize the list of 56 sectors and the list of 44 countries and areas in the WIOD table (2016 release version).

\begin{tabular}{|c|c|c|}
\hline no. & sector name & "sector code \\
\hline 1 & Crop and animal production, hunting and related service activities & A01 \\
\hline 2 & Forestry and logging & A02 \\
\hline 3 & Fishing and aquaculture & A03 \\
\hline 4 & Mining and quarrying & $\mathrm{B}$ \\
\hline 5 & Manufacture of food products, beverages and tobacco products & $\mathrm{C} 10-\mathrm{C} 12$ \\
\hline 6 & Manufacture of textiles, wearing apparel and leather products & $\mathrm{C} 13-\mathrm{C} 15$ \\
\hline 7 & Manufacture of wood and of products of wood and cork, except furniture; manufacture of articles of straw and plaiting materials & C16 \\
\hline 8 & Manufacture of paper and paper products & $\mathrm{C} 17$ \\
\hline 9 & Printing and reproduction of recorded media & $\mathrm{C} 18$ \\
\hline 10 & Manufacture of coke and refined petroleum products & $\mathrm{C} 19$ \\
\hline 11 & Manufacture of chemicals and chemical products & $\mathrm{C} 20$ \\
\hline 12 & Manufacture of basic pharmaceutical products and pharmaceutical preparations & $\mathrm{C} 21$ \\
\hline 13 & Manufacture of rubber and plastic products & $\mathrm{C} 22$ \\
\hline 14 & Manufacture of other non-metallic mineral products & $\mathrm{C} 23$ \\
\hline 15 & Manufacture of basic metals & $\mathrm{C} 24$ \\
\hline 16 & Manufacture of fabricated metal products, except machinery and equipment & $\mathrm{C} 25$ \\
\hline 17 & Manufacture of computer, electronic and optical products & $\mathrm{C} 26$ \\
\hline 18 & Manufacture of electrical equipment & $\mathrm{C} 27$ \\
\hline 19 & Manufacture of machinery and equipment n.e.c. & $\mathrm{C} 28$ \\
\hline 20 & Manufacture of motor vehicles, trailers and semi-trailers & $\mathrm{C} 29$ \\
\hline 21 & Manufacture of other transport equipment & $\mathrm{C} 30$ \\
\hline 22 & Manufacture of furniture; other manufacturing & $\mathrm{C} 31 \mathrm{C} 32$ \\
\hline 23 & Repair and installation of machinery and equipment & $\mathrm{C} 33$ \\
\hline 24 & Electricity, gas, steam and air conditioning supply & D35 \\
\hline 25 & Water collection, treatment and supply & E36 \\
\hline 26 & Sewerage; waste collection, treatment and disposal activities; materials recovery; remediation activities and other waste management services & E37-E39 \\
\hline 27 & Construction & $\mathrm{F}$ \\
\hline 28 & Wholesale and retail trade and repair of motor vehicles and motorcycles & G45 \\
\hline 29 & Wholesale trade, except of motor vehicles and motorcycles & G46 \\
\hline 30 & Retail trade, except of motor vehicles and motorcycles & G47 \\
\hline 31 & Land transport and transport via pipelines & H49 \\
\hline 32 & Water transport & H50 \\
\hline 33 & Air transport & H51 \\
\hline 34 & Warehousing and support activities for transportation & $\mathrm{H} 52$ \\
\hline 35 & Postal and courier activities & H53 \\
\hline 36 & Accommodation and food service activities & I \\
\hline 37 & Publishing activities & $\mathrm{J} 58$ \\
\hline 38 & Motion picture, video and television programme production, sound recording and music publishing activities; programming and broadcasting activities & J59 J60 \\
\hline 39 & Telecommunications & $\mathrm{J} 61$ \\
\hline 40 & Computer programming, consultancy and related activities; information service activities & J62 J63 \\
\hline 41 & Financial service activities, except insurance and pension funding & K64 \\
\hline 42 & Insurance, reinsurance and pension funding, except compulsory social security & K65 \\
\hline 43 & Activities auxiliary to financial services and insurance activities & K66 \\
\hline 44 & Real estate activities & L68 \\
\hline 45 & Legal and accounting activities; activities of head offices; management consultancy activities & M69 M70 \\
\hline 46 & Architectural and engineering activities; technical testing and analysis & M71 \\
\hline 47 & Scientific research and development & M72 \\
\hline 48 & Advertising and market research & M73 \\
\hline 49 & Other professional, scientific and technical activities; veterinary activities & M74 M75 \\
\hline 50 & Administrative and support service activities & $\mathrm{N}$ \\
\hline 51 & Public administration and defence; compulsory social security & O84 \\
\hline 52 & Education & P85 \\
\hline 53 & Human health and social work activities & Q \\
\hline 54 & Other service activities & RS \\
\hline 55 & Activities of households as employers; undifferentiated goods- and services-producing activities of households for own use & $\mathrm{T}$ \\
\hline 56 & Activities of extraterritorial organizations and bodies & $\mathrm{U}$ \\
\hline
\end{tabular}

Table B.1: List of 56 sectors in each of 44 countries in the WIOD table. 


\begin{tabular}{|c|c|c|}
\hline no & country name & country code \\
\hline 1 & Australia & AUS \\
\hline 2 & Austria & AUT \\
\hline 3 & Belgium & BEL \\
\hline 4 & Bulgaria & BGR \\
\hline 5 & Brazil & BRA \\
\hline 6 & Canada & $\mathrm{CAN}$ \\
\hline 7 & Switzerland & $\mathrm{CHE}$ \\
\hline 8 & China & $\mathrm{CHN}$ \\
\hline 9 & Cyprus & CYP \\
\hline 10 & Czech Republic & $\mathrm{CZE}$ \\
\hline 11 & Germany & DEU \\
\hline 12 & Denmark & DNK \\
\hline 13 & Spain & ESP \\
\hline 14 & Estonia & EST \\
\hline 15 & Finland & FIN \\
\hline 16 & France & FRA \\
\hline 17 & United Kingdom & GBR \\
\hline 18 & Greece & GRC \\
\hline 19 & Croatia & $\mathrm{HRV}$ \\
\hline 20 & Hungary & HUN \\
\hline 21 & Indonesia & IDN \\
\hline 22 & India & IND \\
\hline 23 & Ireland & IRL \\
\hline 24 & Italy & ITA \\
\hline 25 & Japan & JPN \\
\hline 26 & Korea & KOR \\
\hline 27 & Lithuania & LTU \\
\hline 28 & Luxembourg & LUX \\
\hline 29 & Latvia & LVA \\
\hline 30 & Mexico & MEX \\
\hline 31 & Malta & MLT \\
\hline 32 & Netherlands & NLD \\
\hline 33 & Norway & NOR \\
\hline 34 & Poland & POL \\
\hline 35 & Portugal & PRT \\
\hline 36 & Romania & ROU \\
\hline 37 & Russian Federation & RUS \\
\hline 38 & Slovak Republic & SVK \\
\hline 39 & Slovenia & SVN \\
\hline 40 & Sweden & SWE \\
\hline 41 & Turkey & TUR \\
\hline 42 & Taiwan & TWN \\
\hline 43 & United States & USA \\
\hline 44 & Rest of the World & ROW \\
\hline
\end{tabular}

Table B.2: List of 44 countries (nations) and areas in the WIOD table. 


\begin{tabular}{rrrrrr}
\hline \hline & mean & median & max & min & skewness \\
\hline 2000 & 4.14 & 0.00036 & 108243.68 & 0.00 & 194.82 \\
2001 & 4.09 & 0.00040 & 112267.14 & 0.00 & 196.01 \\
2002 & 4.19 & 0.00048 & 107060.84 & 0.00 & 196.77 \\
2003 & 4.74 & 0.00070 & 122185.72 & 0.00 & 199.23 \\
2004 & 5.46 & 0.00098 & 137786.65 & 0.00 & 204.64 \\
2005 & 6.14 & 0.00120 & 153480.65 & 0.00 & 218.59 \\
2006 & 6.81 & 0.00144 & 177839.87 & 0.00 & 226.11 \\
2007 & 7.85 & 0.00194 & 224846.10 & 0.00 & 240.24 \\
2008 & 8.90 & 0.00229 & 292710.91 & 0.00 & 281.04 \\
2009 & 8.00 & 0.00203 & 269025.66 & 0.00 & 279.77 \\
2010 & 9.00 & 0.00205 & 295837.80 & 0.00 & 300.23 \\
2011 & 10.34 & 0.00241 & 378726.83 & 0.00 & 337.85 \\
2012 & 10.63 & 0.00235 & 444664.66 & 0.00 & 345.17 \\
2013 & 11.10 & 0.00242 & 509681.09 & 0.00 & 362.35 \\
2014 & 11.45 & 0.00243 & 541342.11 & 0.00 & 367.45 \\
\hline \hline
\end{tabular}

Table B.3: Descriptive statistics for the weights (traded amounts in millions of dollars) among different 2464 industries from 44 countries over the period 2000-2014. Mathematically, the weights are the elements of the $W^{\text {supra }}$ matrix. 


\section{Appendix C. Basic network properties of the global input-output network}

Trade diversity and concentration from the total degrees and strengths of nodes:

Figure C.1 shows the distributions of the total degrees and strengths of 2464 industries from 44 countries based on the original input-output table in 2014. Each degree indicates the total number of trading partners while each strength represents the total volume of trades for a particular sector. Note that we also distinguish the in-coming degrees (strengths) and the out-going degrees (strengths) to illustrate input and output dependencies, respectively. Further descriptive statistics for the degrees and strengths of sectors in different years are reported in Tables C.1 and C.2.

It is interesting to observe that, in the binary version, almost all of nodes have degrees larger than 1500 and a negative skewness, i.e. a longer tail on the left side of the distribution, is observed (see Figure C.1 (a) and (b)). This implies that, overall, industries have a relatively diversified list of trading partners from different countries. This is consistent with the fact that the binary version of the network is very dense, for both intra-country and inter-country links.

In contrast, when the intensity of trading relationships is taken into account in the weighted version, as can be seen from Figure C.1 (c) and (d), both total in-strengths and out-strengths exhibit a rightskewed distribution, where only few nodes have strengths much larger than those of the rest. To further investigate the overall heterogeneity level of trading intensities across partners for each sector, we compute the Herfindahl-Hirschman (H-H) indices (e.g., see Kvålseth (2018); Hirschman (1964)) associated with total in- and out-strengths, which are, respectively, defined as

$$
h_{i, i n}^{[\alpha]}=\sum_{j, \beta}\left(w_{j i}^{[\alpha, \beta]} / s_{i, \text { in }}^{[\alpha, \text { total }]}\right)^{2} \quad \text { and } \quad h_{i, \text { out }}^{[\alpha]}=\sum_{j, \beta}\left(w_{i j}^{[\alpha, \beta]} / s_{i, \text { in }}^{[\alpha, \text { total }]}\right)^{2} .
$$

Generally speaking, the $\mathrm{H}-\mathrm{H}$ indices defined in C.1 will range from $1 / N_{\text {supra }}=1 /(N L)$ to 1 , and a larger value indicates a higher level of the concentration (or specialization) of the inputs (purchased from fewer seller-sectors) or outputs (distributed across limited buyer-sectors).

In our work, we find that while almost all industries in different countries have a moderate or negligible level of concentration of trading across partners, some other industries do intensively trade with few input suppliers or output customers in the global production network (see Figure C.3 and the list of the top 100 sectors that have highest $\mathrm{H}-\mathrm{H}$ concentration levels in Figure C.4. Digging deeper, we find that indeed these less quantitatively diversified sectors come from different countries with various industrial codes, hence no overall definite conclusion can be drawn. However, since they are more concentrated with a limited number of trading partners, they may not be very active in the large international industrial clusters that will be discussed later. 


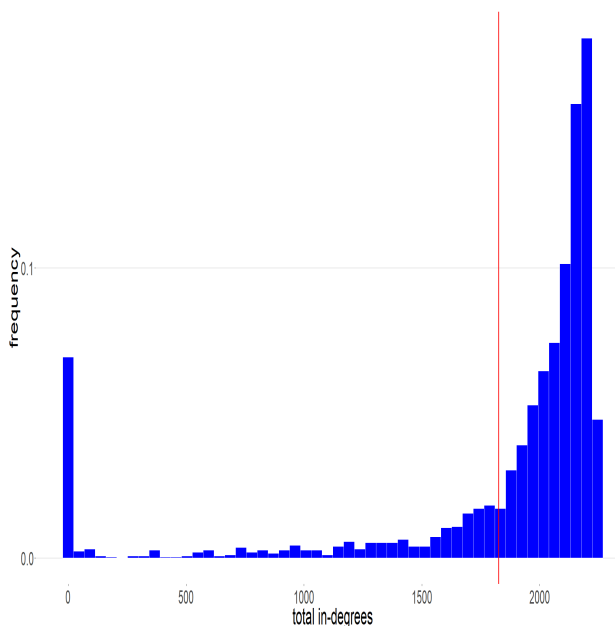

(a)

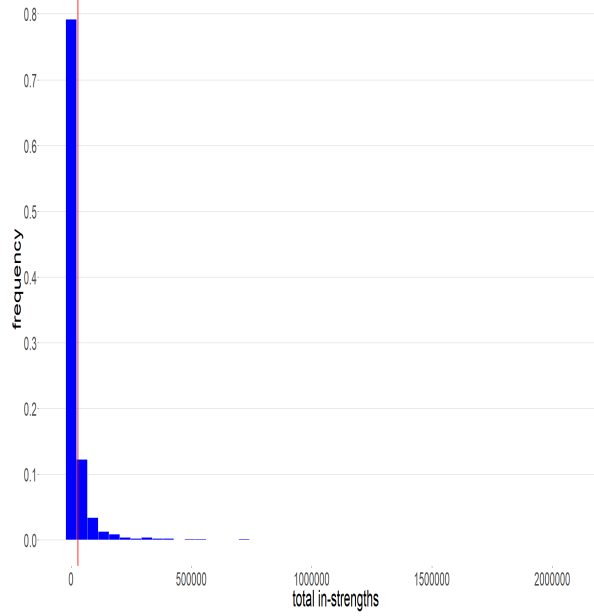

(c)

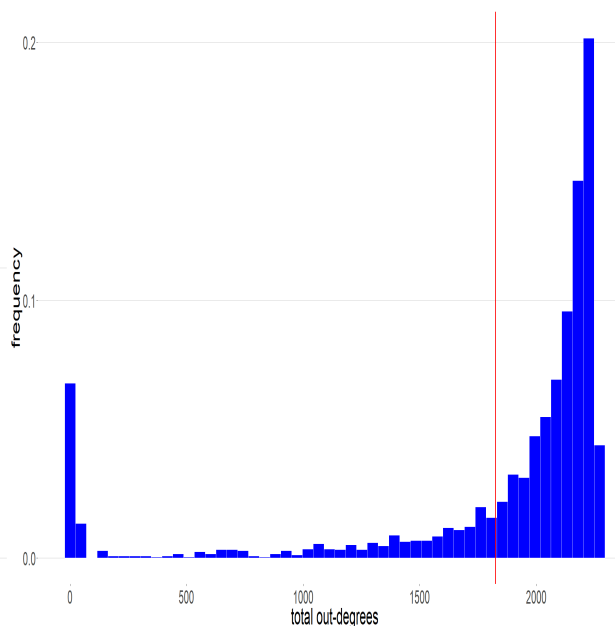

(b)

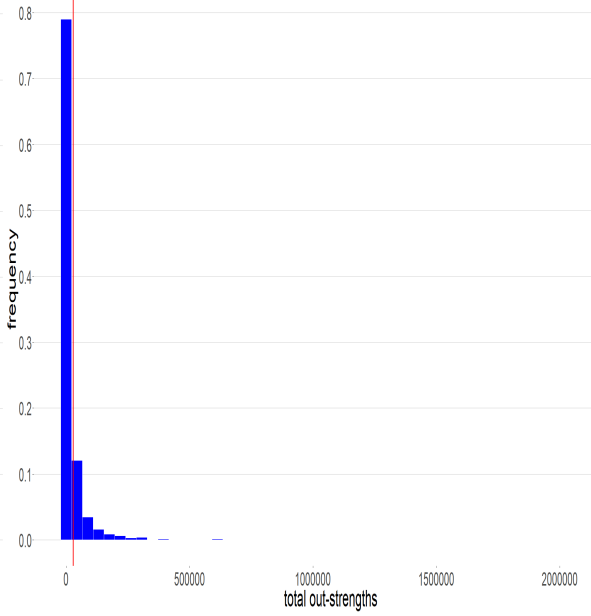

(d)

Figure C.1: Distributions of total in- and out-degrees (panels (a) and (b)) and distributions of total in- and outstrengths (panels (c) and (d)). The red vertical line indicates the mean value. 


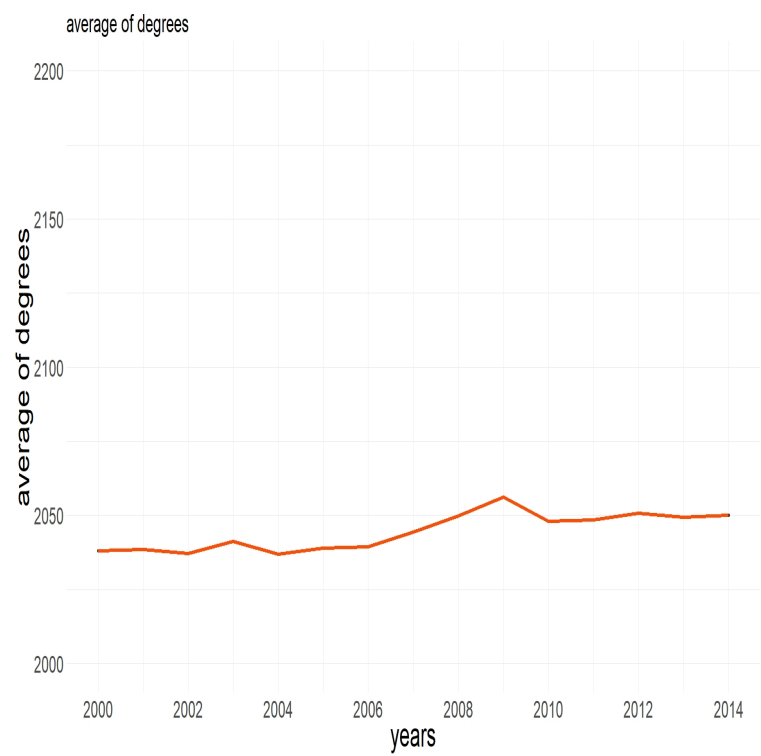

(a) The average of degrees

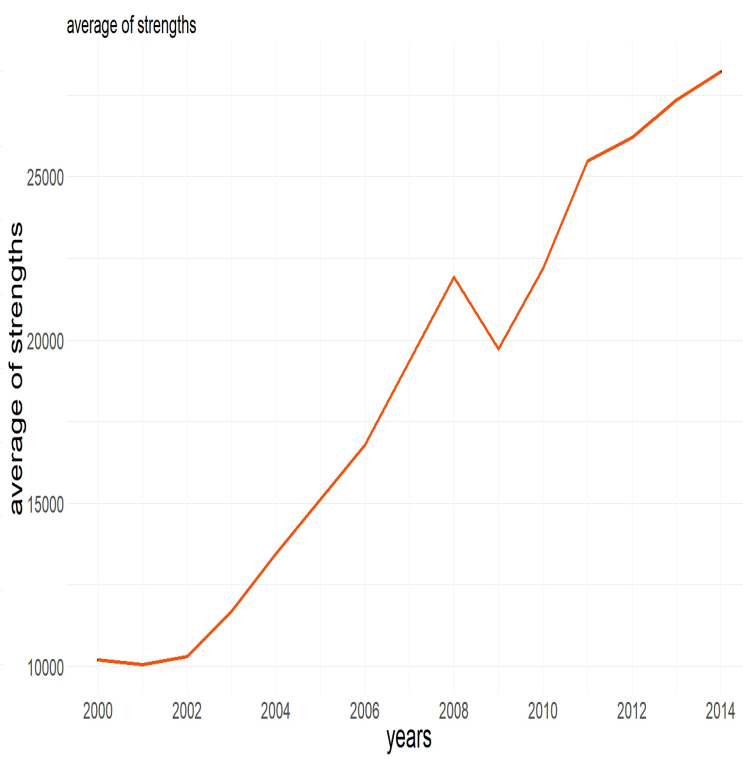

(b) The average of strengths

Figure C.2: The average of degrees (numbers of trading partners) and the average of the strengths (the total traded amounts in millions of dollars) of industries over the period 2000-2014.

\begin{tabular}{lllllllllll}
\hline \hline & \multicolumn{4}{c}{ out-degree } & \multicolumn{4}{c}{ in-dregree } \\
& mean & median & $\max$ & $\min$ & skewness & mean & median & max & min & skewness \\
\hline 2000 & 2038.14 & 2292.00 & 2294.00 & 0.00 & -2.59 & 2038.14 & 2198.00 & 2251.00 & 0.00 & -3.36 \\
2001 & 2038.57 & 2293.00 & 2295.00 & 0.00 & -2.59 & 2038.57 & 2198.00 & 2251.00 & 0.00 & -3.37 \\
2002 & 2037.24 & 2293.00 & 2295.00 & 0.00 & -2.58 & 2037.24 & 2197.00 & 2250.00 & 0.00 & -3.37 \\
2003 & 2041.16 & 2293.00 & 2295.00 & 0.00 & -2.61 & 2041.16 & 2201.00 & 2254.00 & 0.00 & -3.37 \\
2004 & 2036.86 & 2294.00 & 2297.00 & 0.00 & -2.57 & 2036.86 & 2196.00 & 2248.00 & 0.00 & -3.38 \\
2005 & 2038.90 & 2293.00 & 2295.00 & 0.00 & -2.60 & 2038.90 & 2198.00 & 2250.00 & 0.00 & -3.36 \\
2006 & 2039.37 & 2294.00 & 2296.00 & 0.00 & -2.59 & 2039.37 & 2198.00 & 2247.00 & 0.00 & -3.38 \\
2007 & 2044.27 & 2294.00 & 2296.00 & 0.00 & -2.63 & 2044.27 & 2202.00 & 2255.00 & 0.00 & -3.37 \\
2008 & 2049.89 & 2295.00 & 2297.00 & 0.00 & -2.66 & 2049.89 & 2208.00 & 2256.00 & 0.00 & -3.39 \\
2009 & 2056.17 & 2294.00 & 2296.00 & 0.00 & -2.71 & 2056.17 & 2215.00 & 2258.00 & 0.00 & -3.39 \\
2010 & 2047.98 & 2294.00 & 2297.00 & 0.00 & -2.64 & 2047.98 & 2206.00 & 2257.00 & 0.00 & -3.39 \\
2011 & 2048.43 & 2295.00 & 2297.00 & 0.00 & -2.64 & 2048.43 & 2204.00 & 2254.00 & 0.00 & -3.40 \\
2012 & 2050.75 & 2295.00 & 2297.00 & 0.00 & -2.66 & 2050.75 & 2207.00 & 2257.00 & 0.00 & -3.40 \\
2013 & 2049.36 & 2295.00 & 2297.00 & 0.00 & -2.64 & 2049.36 & 2205.00 & 2256.00 & 0.00 & -3.40 \\
2014 & 2050.11 & 2295.00 & 2297.00 & 0.00 & -2.65 & 2050.11 & 2206.00 & 2259.00 & 0.00 & -3.40 \\
\hline \hline
\end{tabular}

Table C.1: Descriptive statistics for the total in-degrees (in-deg.) and total out-degrees (out-deg.) of industries of different countries over the period 2000-2014. These degrees indicate the total number of input providers and output customers of industries, respectively. 


\begin{tabular}{rrrrrrrrrrr}
\hline \hline \multicolumn{1}{c}{ out-strength } & \multicolumn{3}{c}{ in-strength } \\
& mean & median & max & min & skewness & mean & median & max & min & skewness \\
\hline 2000 & 10203.69 & 1482.62 & 520662.94 & 0.00 & 7.76 & 10203.69 & 1528.46 & 601310.93 & 0.00 & 8.11 \\
2001 & 10067.04 & 1505.09 & 520018.16 & 0.00 & 7.89 & 10067.04 & 1544.44 & 662165.45 & 0.00 & 8.94 \\
2002 & 10318.20 & 1683.61 & 516178.66 & 0.00 & 8.04 & 10318.20 & 1703.52 & 710021.48 & 0.00 & 9.53 \\
2003 & 11678.59 & 1968.91 & 550096.73 & 0.00 & 7.81 & 11678.59 & 2018.69 & 757248.20 & 0.00 & 9.23 \\
2004 & 13460.95 & 2312.89 & 590936.72 & 0.00 & 7.74 & 13460.95 & 2366.31 & 814150.19 & 0.00 & 8.76 \\
2005 & 15119.28 & 2581.88 & 829788.19 & 0.00 & 8.56 & 15119.28 & 2621.40 & 880764.57 & 0.00 & 8.49 \\
2006 & 16778.55 & 2922.45 & 1036754.48 & 0.00 & 9.03 & 16778.55 & 2958.67 & 948754.53 & 0.00 & 8.25 \\
2007 & 19347.83 & 3492.05 & 1175484.54 & 0.00 & 8.72 & 19347.83 & 3500.86 & 1006047.59 & 0.00 & 7.69 \\
2008 & 21928.81 & 3895.32 & 1693442.94 & 0.00 & 10.71 & 21928.81 & 4006.56 & 1094105.94 & 0.00 & 7.73 \\
2009 & 19717.81 & 3399.06 & 1208091.06 & 0.00 & 8.31 & 19717.81 & 3453.91 & 1119248.06 & 0.00 & 9.00 \\
2010 & 22184.88 & 3497.00 & 1589086.09 & 0.00 & 9.37 & 22184.88 & 3579.50 & 1250019.40 & 0.00 & 8.67 \\
2011 & 25484.10 & 3932.58 & 2105071.02 & 0.00 & 10.77 & 25484.10 & 4021.78 & 1561448.76 & 0.00 & 8.62 \\
2012 & 26201.51 & 3789.64 & 2237154.63 & 0.00 & 10.77 & 26201.51 & 3758.29 & 1772866.98 & 0.00 & 9.09 \\
2013 & 27347.11 & 3930.67 & 2183110.41 & 0.00 & 9.91 & 27347.11 & 3852.11 & 1995572.56 & 0.00 & 9.46 \\
2014 & 28209.77 & 4011.40 & 2145115.96 & 0.00 & 9.44 & 28209.77 & 3847.23 & 2200291.27 & 0.00 & 9.84 \\
\hline \hline
\end{tabular}

Table C.2: Descriptive statistics for the total in-strengths (in-str.) and total out-strengths (out-str.) of industries of different countries over the period 2000-2014. Values of strengths are in millions of dollars. They indicate the total amounts of purchased inputs and sold outputs of industries, respectively.

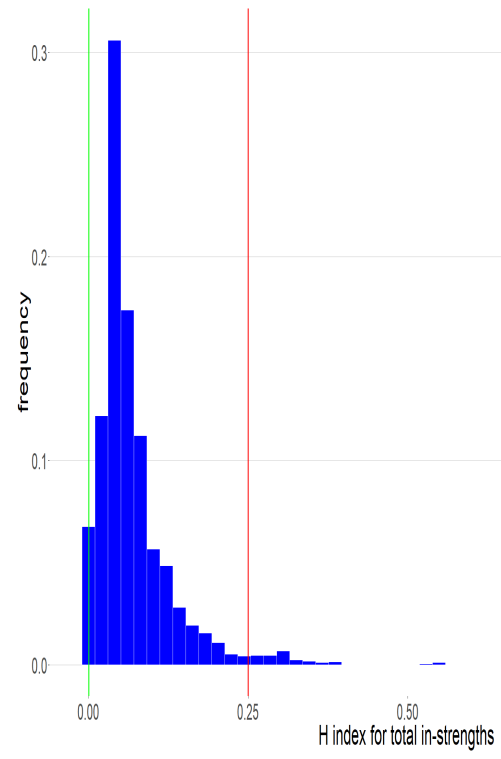

(a)

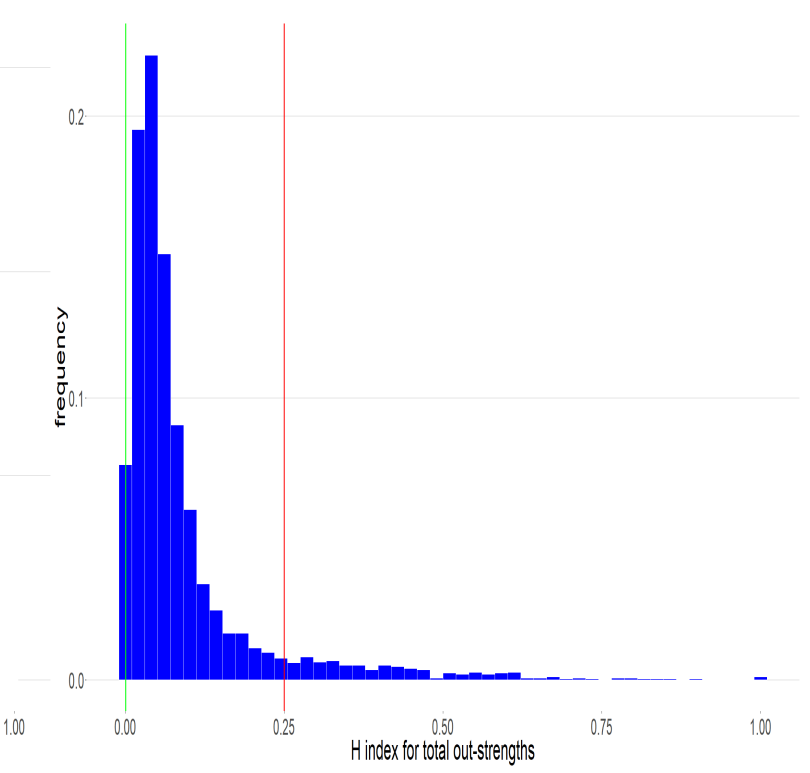

(b)

Figure C.3: Distributions of the H-H indices of total in- and out-strengths. The vertical green line correspond to the minimum $1 / N_{\text {supra }}$. The vertical red line represents the critical value 0.25 , which indicates a high level of concentration if $h>0.25$. 


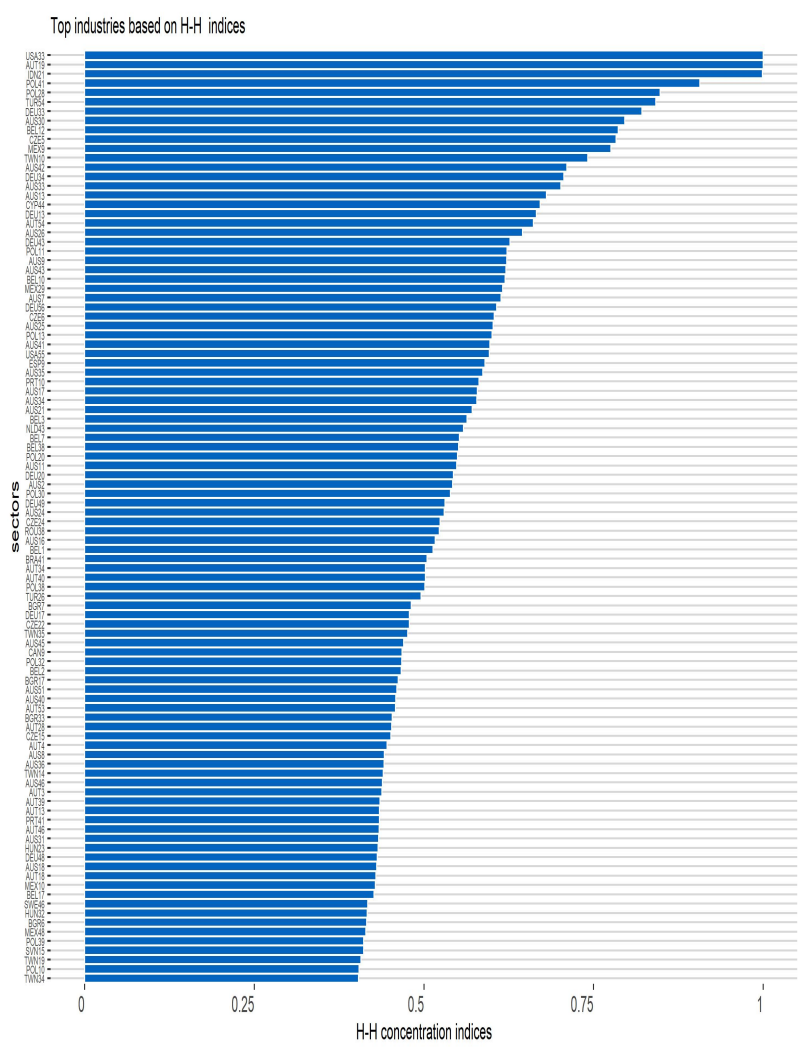

(a) Based on H-H concentration in out-strengths, 2014

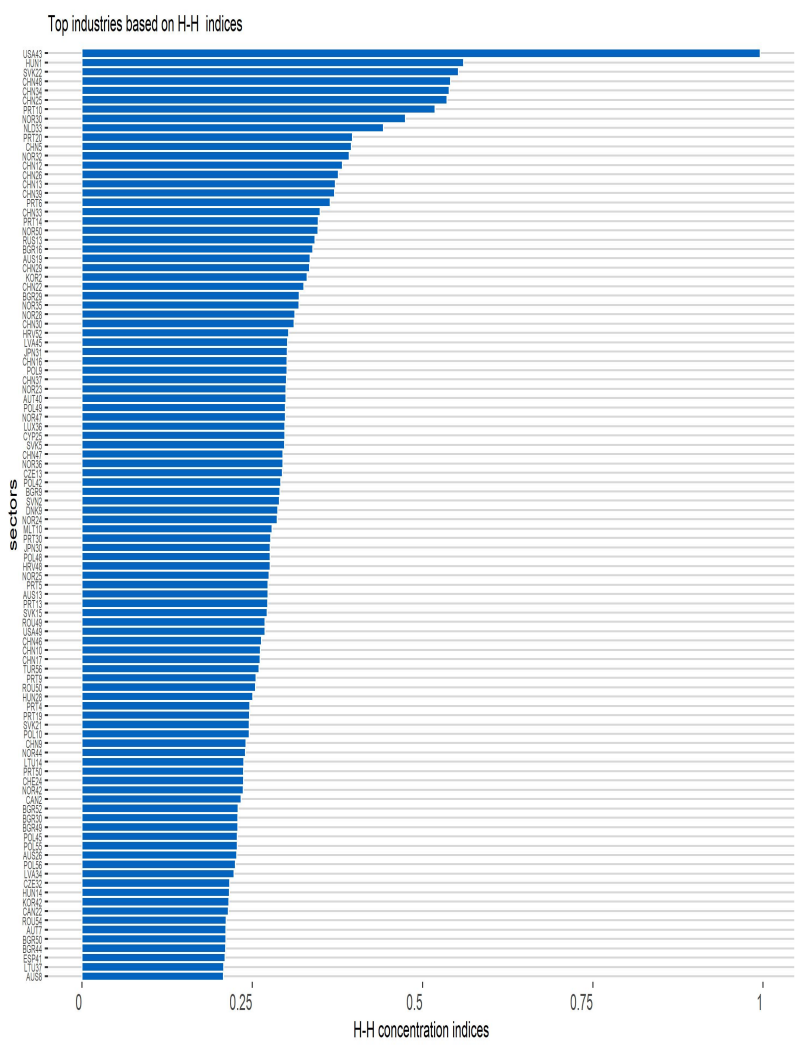

(b) Based on H-H concentration in in-strengths, 2014

Figure C.4: Ranking of top industries based on H-H concentration indices: A larger value indicates a higher level of the concentration of the inputs (purchased from fewer seller-sectors) or outputs (distributed across limited buyer-sectors). Labels on the $y$ axis are given by the country code and by the number related to the sector.

We report the corresponding Pearson correlation coefficients in Figure C.5 to illustrate the overall correlations among different types of node degrees, strengths and the total communicability centralities. First, we can see that the correlation between in-degrees and out-degrees is relatively high. This is not an unexpected result since many nodes have a high level of in- and out-degrees (see Figure C.1 (a) and (b)) and the binary network is very dense. However, the results also show that the correlation levels between degrees and strengths are relatively weaker, which again confirms the distinguished characteristics of the two versions of the network. As stressed before, from the binary analysis we find that on average each sector trades with a relatively diversified list of partners. However, the results from the weighted counterpart point out that the distribution of traded amounts across partners is not homogeneous and several sectors tend to concentrate higher volumes of trades with selected partners.

Furthermore, given the high degree of heterogeneity in the node strengths, it is interesting to observe that interlayer in-strengths and out-strengths are synchronized together, to a certain extent. This implies that, if a sector in a country exports more its outputs, it also purchases more inputs from sectors in other countries and vice versa. Hence, the international diversification in the weighted version seems to be aligned from both the input purchase and output sale perspectives. For a more comprehensive assessment, we also include in Figure C.5 the total receiving and broadcasting communicability centralities defined for the weighted version 
of the network and compute the correlations between them and the various degrees and strengths. As expected, since these two additional measures are able to capture all possible travelling paths between nodes, they are less synchronized with the other lower-order network metrics like the node degrees and strengths.

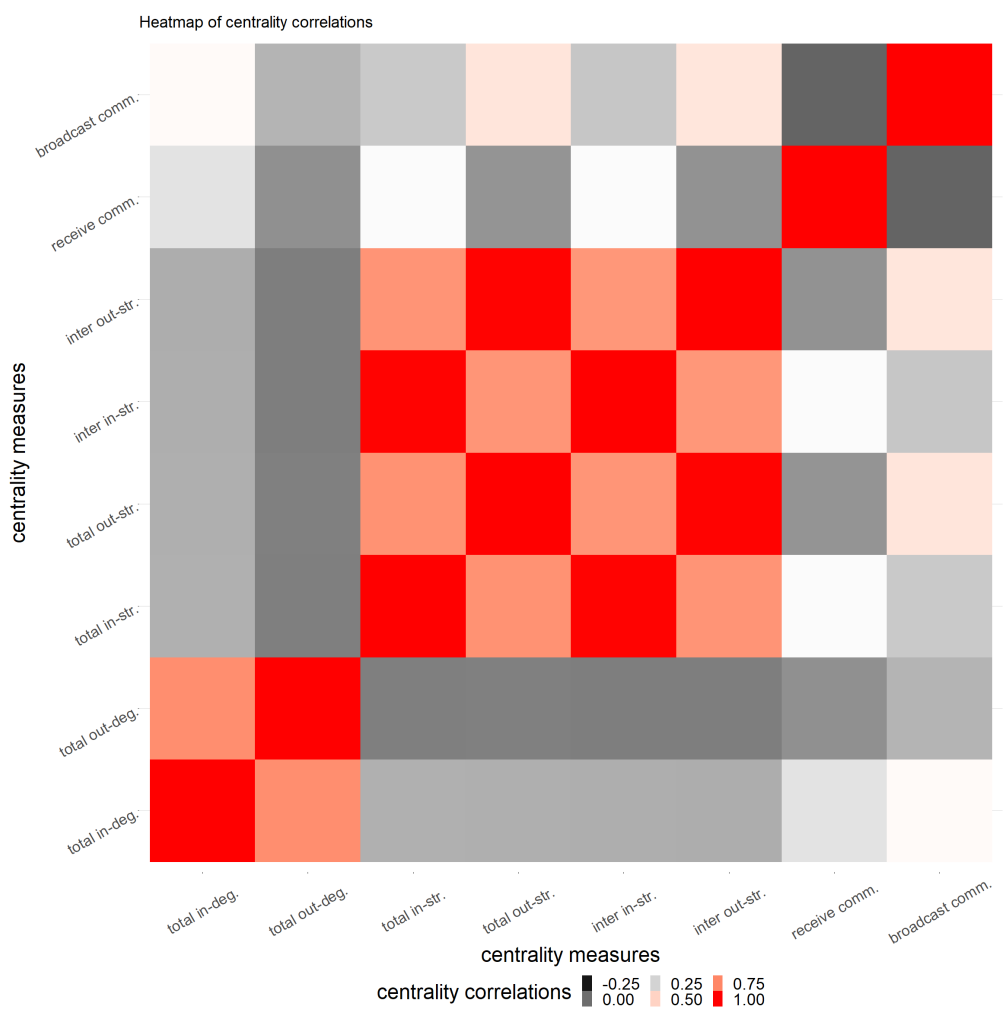

(a)

Figure C.5: Correlations among node degrees, strengths and the total (weighted) communicability centralities. Total

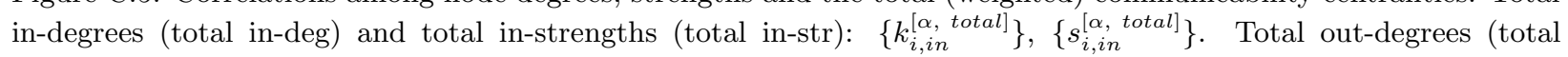
out-deg) and total out-strengths (total out-str): $\left\{k_{i, \text { out }}^{[\alpha, \text { total }]}\right\},\left\{s_{i, \text { out }}^{[\alpha, \text { tal }]}\right\}$. Interlayer in-strengths (inter in-str) and interlayer out-strengths (inter out-str): $\left\{s_{i, \text { in }}^{[\alpha, \text { inter }]}\right\},\left\{s_{i, \text { out }}^{[\alpha, \text { inter }]}\right\}$. The total communicability centralities of a node from/to all nodes in all layers (in the weighted version of the network): receive and broadcast communicability.

\section{Interrelations between layers in a multilayer architecture:}

We shall now reorganize the original input-output table into an equivalent network with a multilayer architecture. In such structure, an intralayer link represents a trade link from a country to another one in a particular sector. An interlayer link, in contrast, implies a trade link from a sector in one country to another sector in the same or another country.

To examine how strongly a layer interacts with another one, we measure the average connectivity and intensity between them. Figure C.6 shows again distinct behaviors in the two facets of the multilayer network. On the one hand, in the binary version, the density of the connections between many layers is very high (see panels (a) and (b) of Figure C.6 19 On the other hand, once the link weights (i.e. the traded amounts) are considered, average intensities are highly heterogeneous. In particular, as shown in panels (c) and (d) of

\footnotetext{
${ }^{19}$ For illustration purpose, in panels (a) and (c) of Figure C.6 we only report the sector indices in the rows and columns of the two color-coded matrices. The list of the corresponding sector names and codes is provided in Table B.1.
} 
Figure C.6. few pairs of layers interact more intensively, suggesting that these industry-based layers trade more between themselves than with the others. We can identify, for example, "Mining and quarrying (B)" and "Manufacture of refined petroleum products (C19)", "Crop and animal production, hunting and related service activities (A01)" and "Manufacture of food products, beverages and tobacco products (C10-C12)", "Manufacture of other non-metallic mineral products (C23)" and "Construction (F)", "Mining and quarrying (B)" and "Electricity, gas, steam and air conditioning supply (D35)" as the couples of industries that, on average, interact more intensively. Intuitively, this illustrates the "natural and universal" input-output dependencies of some industries on important factors of production provided by their counterparts.

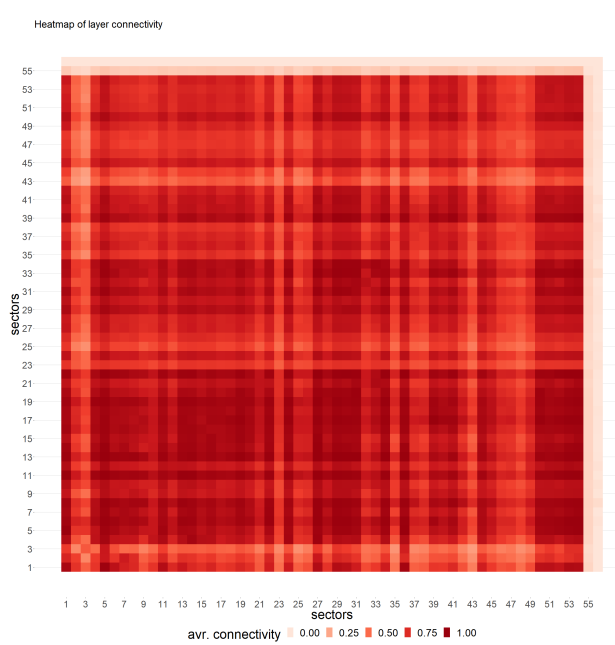

(a)

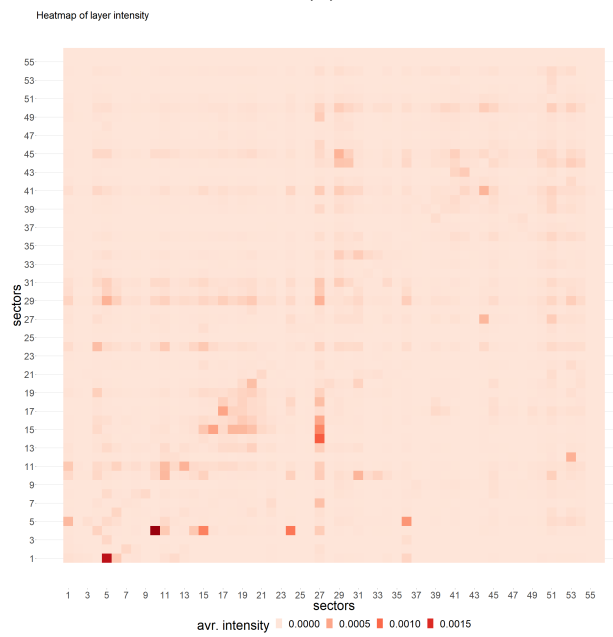

(c)

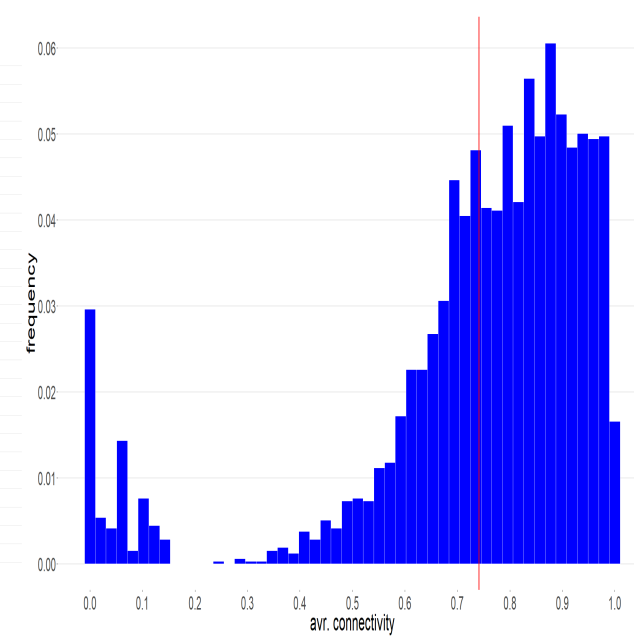

(b)

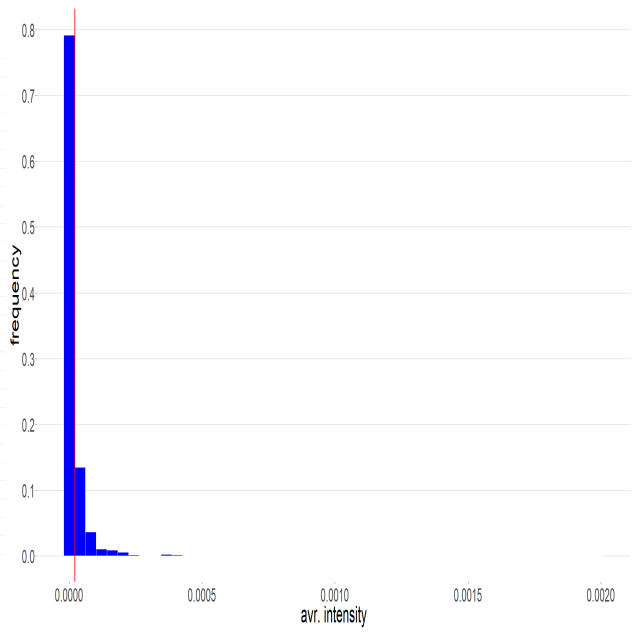

(d)

Figure C.6: Average connectivity and average intensity between layers. The measures for these averages are explained in formulas (4) and (6). The vertical red line in each histogram shows the mean value. Note that here layers represent 56 sectors, while nodes in each layer are 44 countries. The rows and columns of the two color-coded matrices (panel (a) and panel (c)) represent the sector indices. The more detailed list of sector names and codes is reported in Table B.1.

\section{Layer overlaps and correlations.}

Comparing across layers, we want to examine whether layers exhibit similar internal structures. In particular, if trade relations among 44 countries are strong in a particular layer (sector), do they also exist 
and tend to form intensive relations in the other layers (sectors)? To answer this question, we measure the overall overlap coefficient and Pearson correlation coefficient for every pair of layers (Gemmetto \& Garlaschelli, 2014, Gemmetto et al., 2016, Luu \& Lux, 2019). In general, on the one hand, trade relations among 44 countries in binary version are more similar across many couples of layers (see Figure C.7). This is not a very surprising result, since most of countries have a well diversified list of partners when they trade among themselves in each industry. On the other hand, results obtained from the weighted analysis show a more hierarchical structure in which several clusters of layers exhibit a relatively higher level of similarity, while many other couples of layers are much less aligned (see Figures C.7 and C.8 as well as the dendrogram ${ }^{20}$ shown in Figures C.9 and C.10. From this perspective, the way countries trade among themselves is, therefore, not the same in all layers.

\footnotetext{
${ }^{20}$ The analysis of the layer correlation matrices $\left(\left\{R_{b i n}^{[\alpha, \beta]}\right\},\left\{R_{w}^{[\alpha, \beta]}\right\}\right)$ and that of the layer overlap matrices $\left(\left\{O_{b i n}^{[\alpha, \beta]}\right\},\left\{O_{w}^{[\alpha, \beta]}\right\}\right)$ reveal that some layers are more correlated or overlapped. To provide a more detailed refinement and visualization of clustering behaviors among them, we report in Figures C.9 and C.10 the dendrograms obtained from these four matrices. In these figures, the euclidean distance method and average agglomeration method are used to plot the dendrograms.
} 


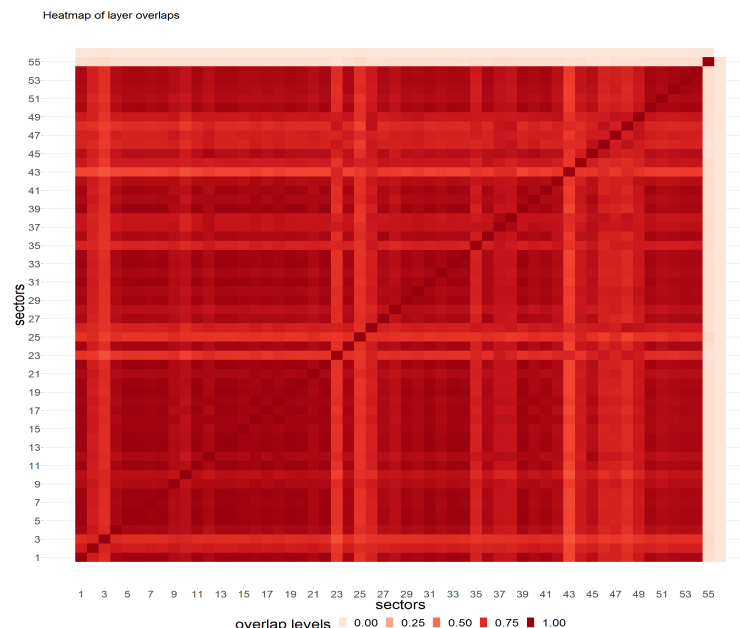

(a)

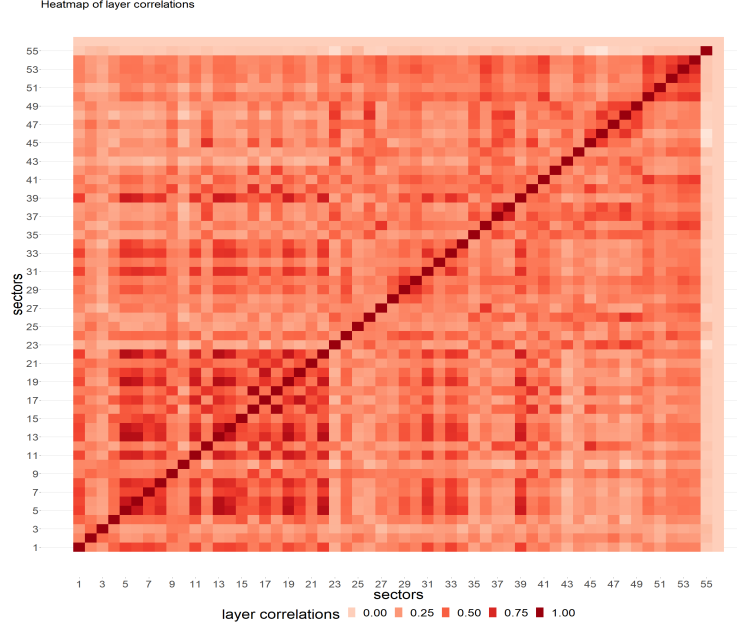

(c)

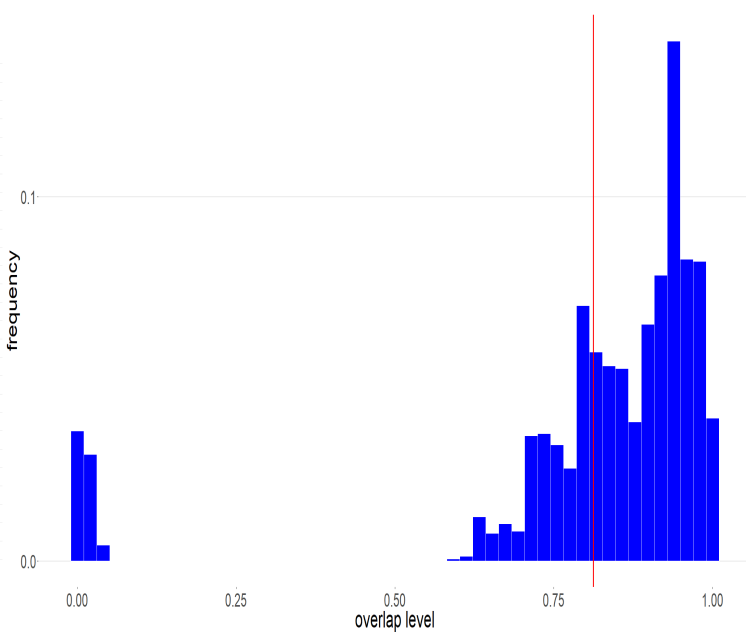

(b)

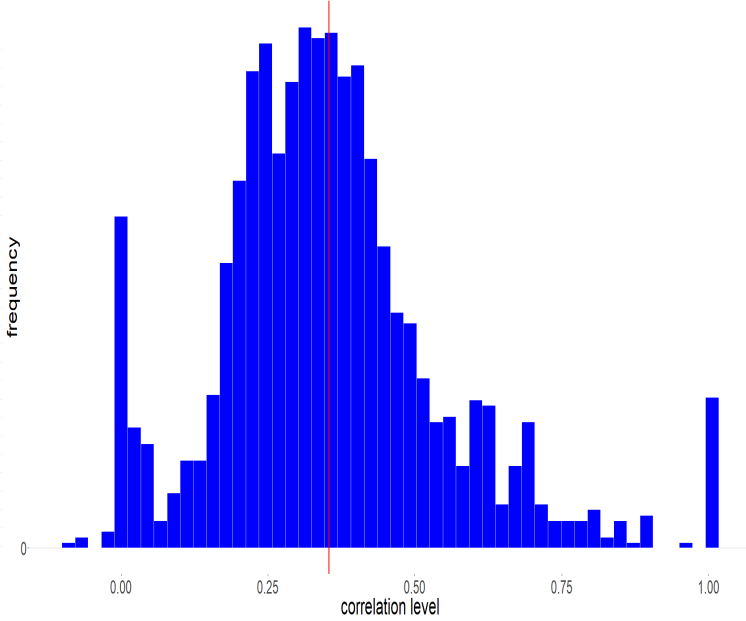

(d)

Figure C.7: Overlaps and correlations between layers in the binary version. The methods used to compute the overlap and correlation coefficients are explained in $(2.2)$. Layers represent 56 sectors, while nodes in each layer are 44 countries. The rows and columns of the two color-coded matrices (panel (a) and panel (c)) represent the sector indices. The more detailed list of sector names and codes is reported in Table B.1. 


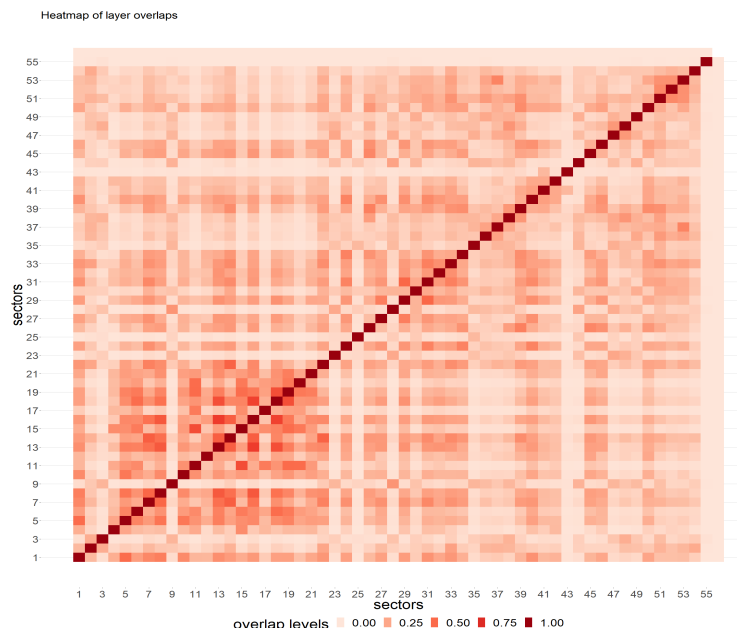

(a)

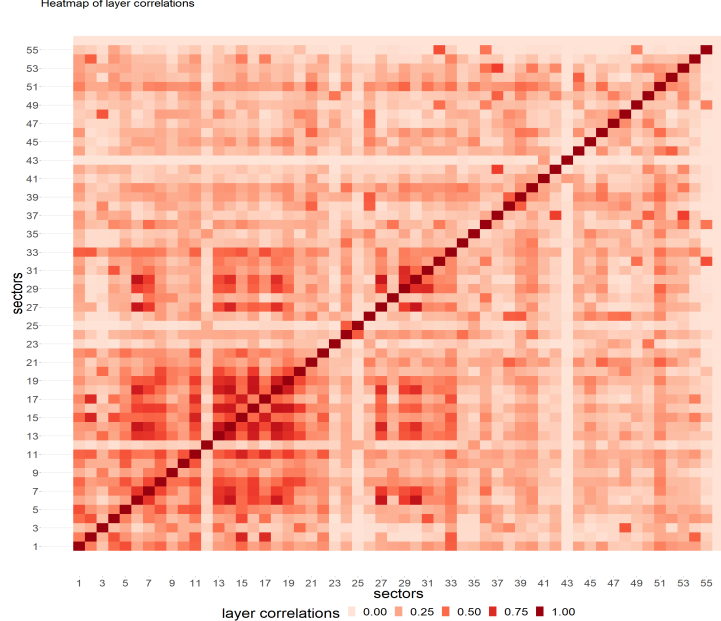

(c)

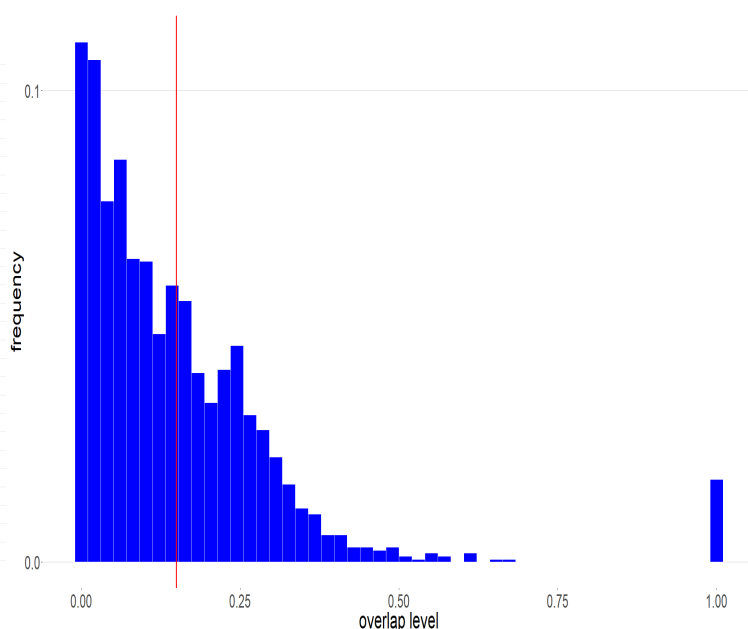

(b)

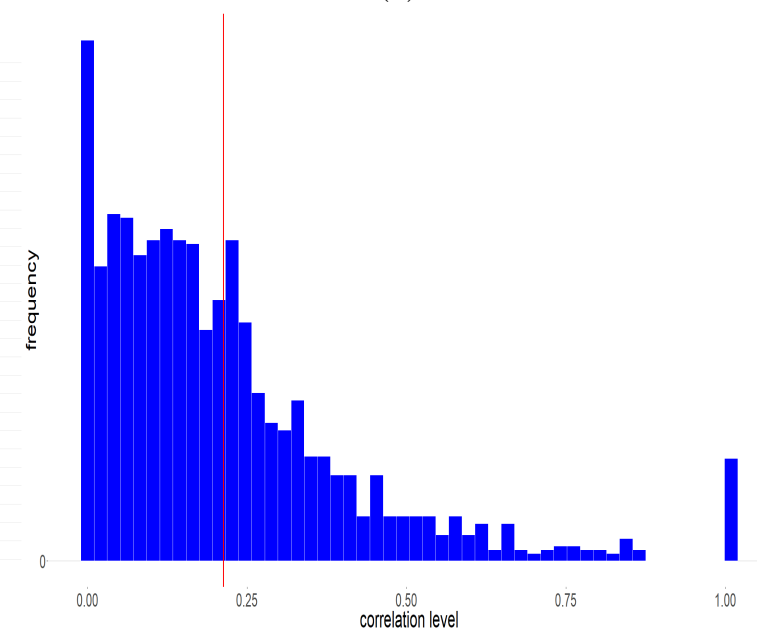

(d)

Figure C.8: Overlaps and correlations between layers in the weighted version. The methods used to compute the overlap and correlation coefficients are explained in (2.2). Layers represent 56 sectors, and nodes in each layer are 44 countries. The rows and columns of the two color-coded matrices (panel (a) and panel (c)) represent the sector indices. The more detailed list of sector names and codes is reported in Table B.1. 


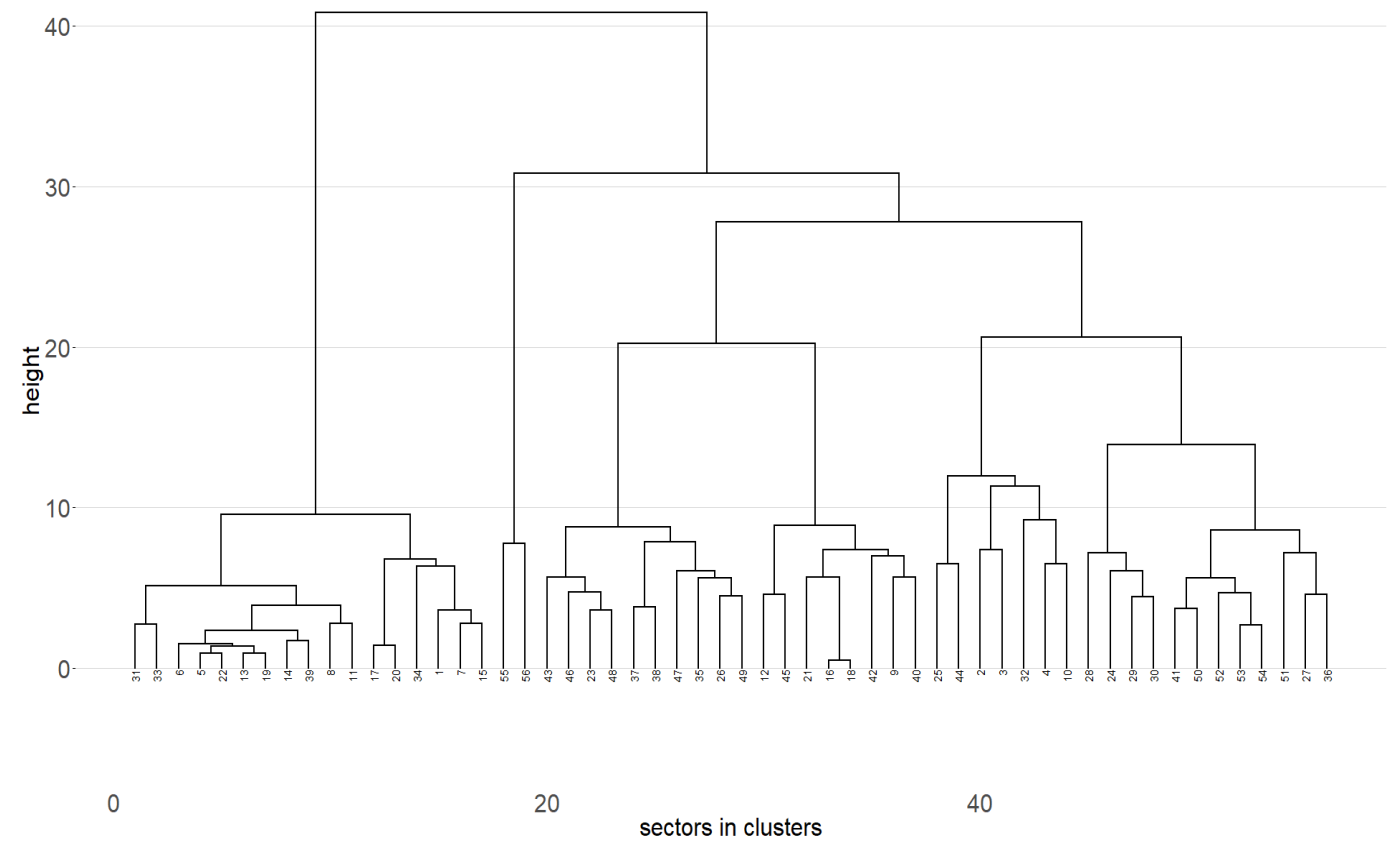

(a) for binary version

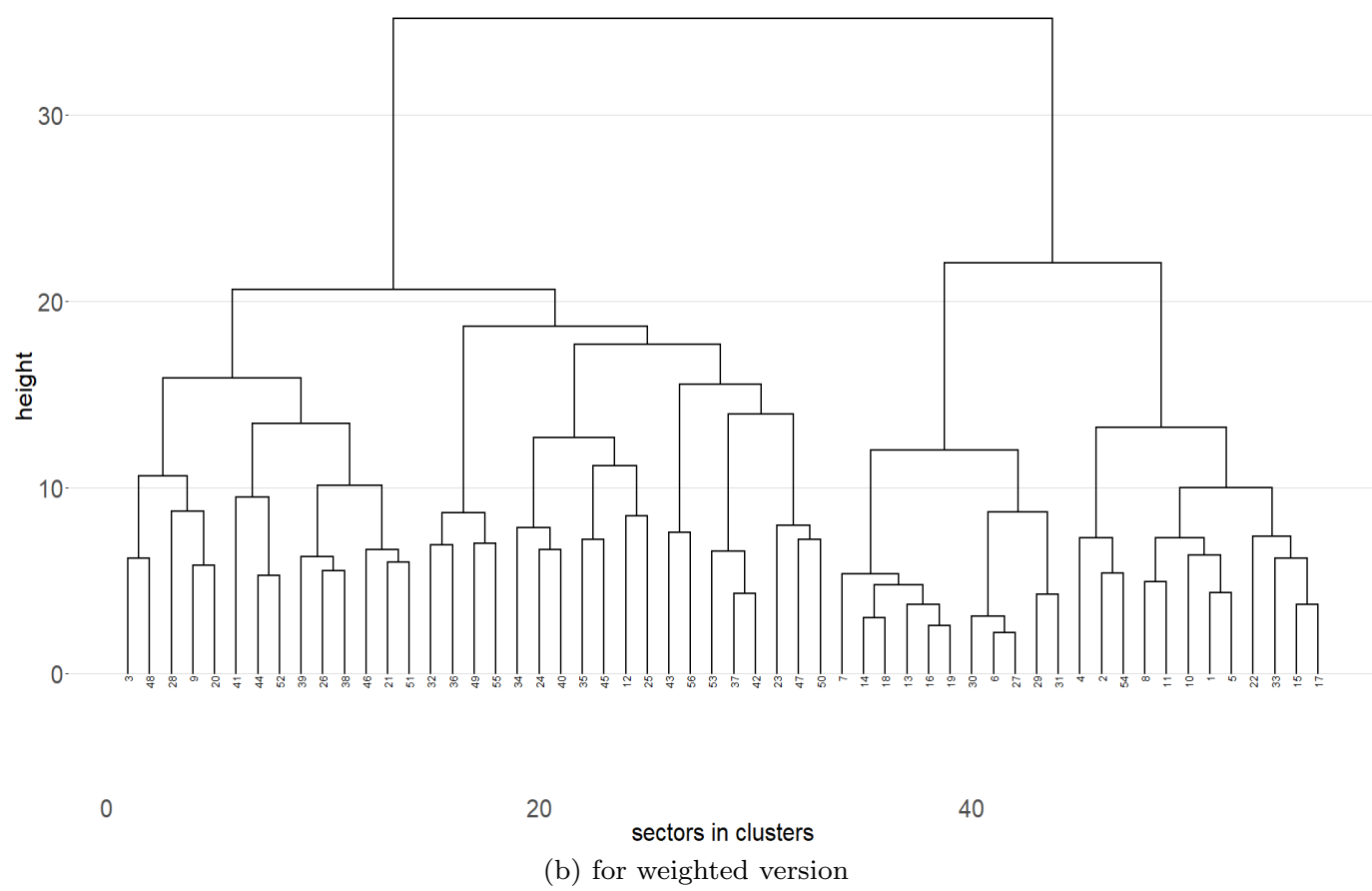

Figure C.9: Dendrogram plot obtained from the layer-layer correlation matrices for the binary version (panel a) and the weighted version (panel (b)). 


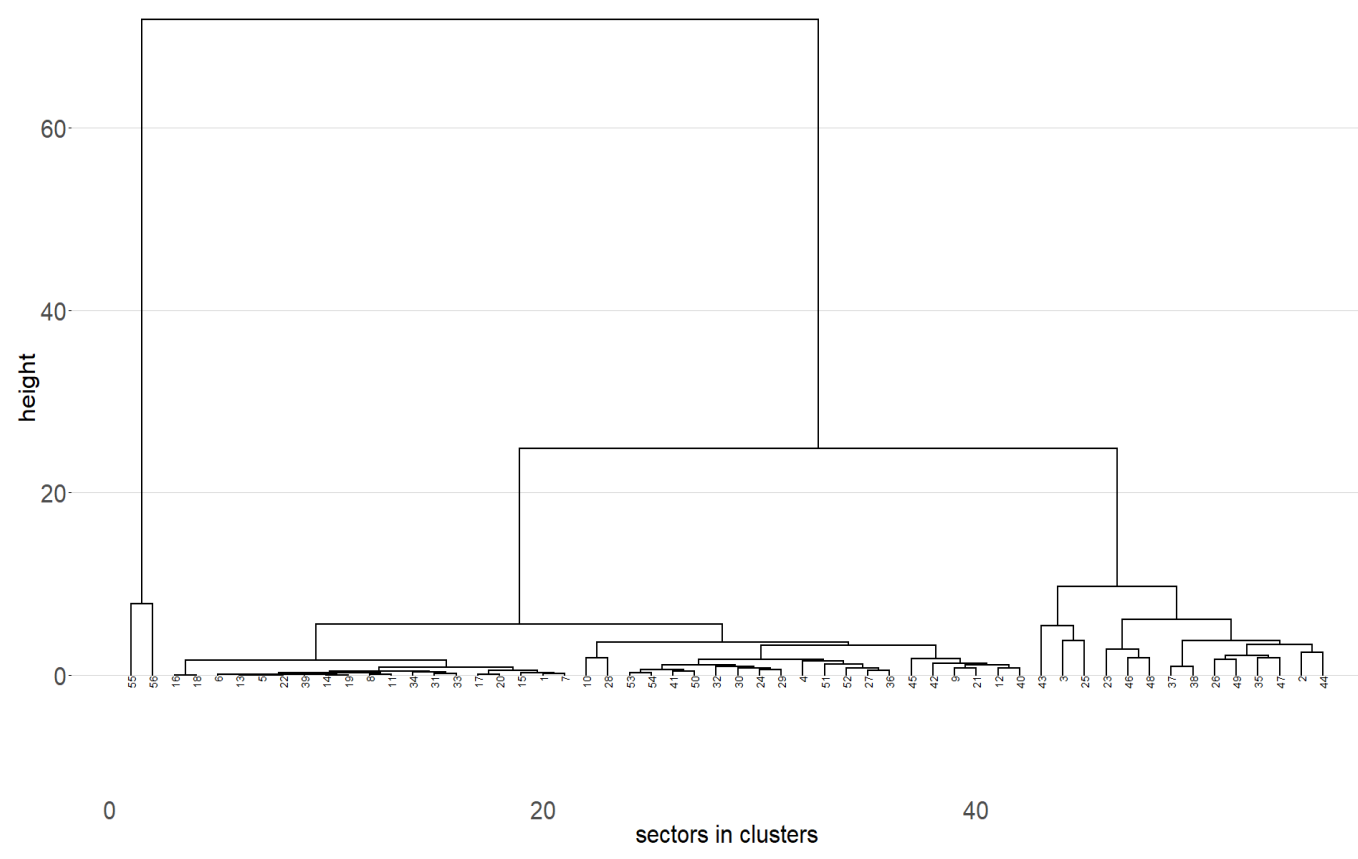

(a) for binary version

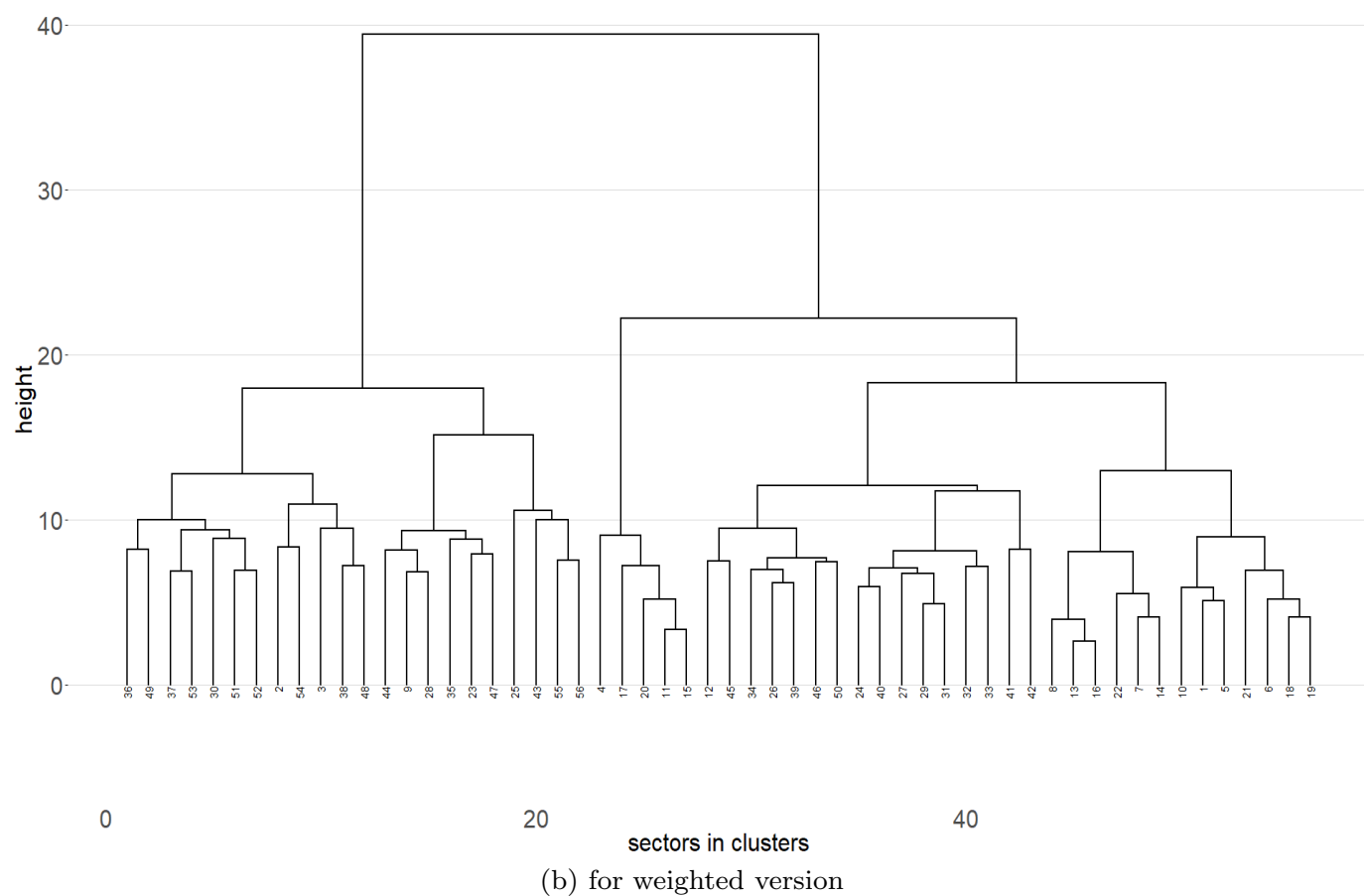

Figure C.10: Dendrogram plot obtained from the layer-layer overlap matrices for the binary version (panel a) and the weighted version (panel (b)).

In summary, our analysis of the basic network properties reveals that the global trade network in inputoutput possesses a complex architecture. The network exhibits a complex and mixed nature of the trade diversification as well as specialization of different countries in different sectors. 
First, binary and the weighted versions have distinct topological features. The results obtained from the binary analysis reveal that a number of industries establish trade links with many partners from different countries. Nevertheless, once the intensity of trade relations is taken into account, we observe a more hierarchical structure of interactions between sectors and countries. The analysis of the weighted version also reveals a heterogeneity in the way they select their input providers and their output customers. These observations are somewhat similar to those found from different empirical analyses of the aggregated World Trade network (see Garlaschelli \& Loffredo, 2005, Serrano et al., 2007, Fagiolo et al., 2008, De Benedictis \& Tajoli, 2011).

In addition, the presence of an already well diversified trading list for many industries and countries in the densely connected binary version suggests that what mainly govern the temporal dynamics of the global trade network in input-output are not the extensive margin effects (i.e. obtaining new partners through new links). Instead, the intensive margin effects (i.e., when the intensities of existing trade linkages in the weighted version are adjusted and/or strengthened over time) may play a more prominent role in explaining the changes in the network structure(Felbermayr \& Kohler, 2006, Helpman et al. 2008). Furthermore, once various types of trade linkages are considered in a multilayer architecture, we find that the interactions between layers vary across pairs of layers in the weighted version. On top of that, although many layers have strongly dissimilar internal structures, few others are somewhat more overlapped or correlated. Therefore, the trade interrelations among countries, while being not homogeneous in general, may span over a certain number of industries. Interestingly, to a certain extent, this property is also similar to what has been found in the multiplex trade network in which each layer is associated with a particular commodity (see Barigozzi et al., 2010, Gemmetto \& Garlaschelli, 2014; Gemmetto et al., 2016). This again confirms a complex and mixed nature of the diversification as well as the specialization of nations in different trading layers (industries or commodities) that cannot be detected if we only consider the aggregated trade network. 


\section{Appendix D. Additional community structure results}

We report some additional tables and figures related to the application of the proposed methodology of community detection.

\begin{tabular}{|c|c|c|c|c|c|}
\hline Countries & $\begin{array}{l}\text { Number of Sectors } \\
\text { in Community } 1\end{array}$ & $\begin{array}{l}\text { Number of Sectors } \\
\text { in Community } 2\end{array}$ & $\begin{array}{l}\text { Number of Sectors } \\
\text { Isolated }\end{array}$ & $\begin{array}{c}\text { Community } \\
\text { with highest } \\
\text { number of sectors }\end{array}$ & $\begin{array}{c}\text { Gini } \\
\text { heterogeneity } \\
\text { Index }\end{array}$ \\
\hline AUS & 33 & 0 & 18 & 1 & $64.9 \%$ \\
\hline AUT & 2 & 1 & 20 & 13 & $72.5 \%$ \\
\hline BEL & 3 & 0 & 18 & 22 & $83.4 \%$ \\
\hline BGR & 41 & 0 & 15 & 1 & $46.4 \%$ \\
\hline BRA & 0 & 0 & 13 & 5 & $46.3 \%$ \\
\hline CAN & 0 & 35 & 16 & 2 & $60.5 \%$ \\
\hline CHE & 2 & 0 & 20 & 14 & $72.6 \%$ \\
\hline CHN & 41 & 1 & 10 & 1 & $46.1 \%$ \\
\hline CYP & 0 & 2 & 23 & 16 & $81.1 \%$ \\
\hline CZE & 0 & 30 & 17 & 2 & $69.9 \%$ \\
\hline $\mathrm{DEU}$ & 37 & 6 & 11 & 1 & $55.1 \%$ \\
\hline DNK & 5 & 0 & 14 & 10 & $62.1 \%$ \\
\hline ESP & 1 & 0 & 10 & 3 & $40.9 \%$ \\
\hline $\mathrm{EST}$ & 0 & 0 & 20 & 18 & $77.5 \%$ \\
\hline FIN & 2 & 0 & 16 & 6 & $53.8 \%$ \\
\hline FRA & 1 & 36 & 10 & 2 & $58.0 \%$ \\
\hline GBR & 8 & 33 & 12 & 2 & $62.9 \%$ \\
\hline GRC & 38 & 0 & 18 & 1 & $54.0 \%$ \\
\hline HRV & 0 & 0 & 16 & 7 & $53.8 \%$ \\
\hline HUN & 0 & 1 & 27 & 24 & $87.7 \%$ \\
\hline IDN & 0 & 0 & 15 & 9 & $58.3 \%$ \\
\hline IND & 36 & 0 & 20 & 1 & $58.7 \%$ \\
\hline IRL & 1 & 12 & 29 & 2 & $94.0 \%$ \\
\hline ITA & 2 & 0 & 11 & 4 & $40.9 \%$ \\
\hline JPN & 39 & 0 & 9 & 1 & $50.2 \%$ \\
\hline KOR & 46 & 0 & 8 & 1 & $32.4 \%$ \\
\hline LTU & 0 & 0 & 23 & 15 & $74.7 \%$ \\
\hline LUX & 0 & 2 & 44 & 39 & $98.7 \%$ \\
\hline LVA & 0 & 0 & 18 & 17 & $76.1 \%$ \\
\hline MEX & 0 & 33 & 17 & 2 & $64.6 \%$ \\
\hline MLT & 0 & 0 & 30 & 25 & $95.0 \%$ \\
\hline NLD & 11 & 2 & 21 & 23 & $80.6 \%$ \\
\hline NOR & 28 & 0 & 19 & 1 & $74.1 \%$ \\
\hline POL & 0 & 45 & 11 & 2 & $35.4 \%$ \\
\hline PRT & 30 & 0 & 14 & 1 & $70.2 \%$ \\
\hline ROU & 0 & 0 & 16 & 19 & $78.3 \%$ \\
\hline RUS & 0 & 28 & 26 & 2 & $74.9 \%$ \\
\hline SVK & 0 & 4 & 19 & 20 & $80.2 \%$ \\
\hline SVN & 0 & 0 & 17 & 12 & $66.8 \%$ \\
\hline SWE & 3 & 0 & 10 & 11 & $63.9 \%$ \\
\hline TUR & 0 & 0 & 18 & 8 & $54.0 \%$ \\
\hline TWN & 2 & 0 & 14 & 21 & $79.3 \%$ \\
\hline USA & 0 & 41 & 12 & 2 & $46.2 \%$ \\
\hline ROW & 31 & 6 & 5 & 1 & $66.9 \%$ \\
\hline
\end{tabular}

Table D.1: In this Table, we report for each country the number of sectors that belong to the two largest communities (1 and 2, respectively), the number of sector that does not belong to any community (i.e. isolated), the indication of the community with the highest number of sectors of that country and the Gini heterogeneity index to measure the dispersion of sectors along the communities in the same country. 


\begin{tabular}{|c|c|c|c|c|c|}
\hline Sectors & $\begin{array}{l}\text { Number of Countries } \\
\text { in Community } 1\end{array}$ & $\begin{array}{l}\text { Number of Countries } \\
\text { in Community } 2\end{array}$ & $\begin{array}{l}\text { Number of Countries } \\
\text { Isolated }\end{array}$ & $\begin{array}{c}\text { Community } \\
\text { with highest } \\
\text { number of countries }\end{array}$ & $\begin{array}{c}\text { Gini } \\
\text { heterogeneity } \\
\text { Index }\end{array}$ \\
\hline $\mathrm{A} 01$ & 10 & 5 & 0 & 1 & $92.0 \%$ \\
\hline $\mathrm{A} 02$ & 8 & 5 & 10 & 1 & $94.3 \%$ \\
\hline A03 & 4 & 1 & 32 & 1 & $98.7 \%$ \\
\hline $\mathrm{B}$ & 21 & 6 & 7 & 1 & $74.8 \%$ \\
\hline $\mathrm{C} 10-\mathrm{C} 12$ & 10 & 5 & 1 & 1 & $92.1 \%$ \\
\hline $\mathrm{C} 13-\mathrm{C} 15$ & 4 & 0 & 35 & 1 & $98.9 \%$ \\
\hline C16 & 9 & 6 & 4 & 1 & $92.7 \%$ \\
\hline $\mathrm{C} 17$ & 6 & 3 & 19 & 1 & $96.8 \%$ \\
\hline $\mathrm{C} 18$ & 8 & 4 & 6 & 1 & $94.5 \%$ \\
\hline C19 & 17 & 6 & 8 & 1 & $82.4 \%$ \\
\hline $\mathrm{C} 20$ & 11 & 5 & 16 & 1 & $91.8 \%$ \\
\hline $\mathrm{C} 21$ & 9 & 4 & 18 & 1 & $94.3 \%$ \\
\hline $\mathrm{C} 22$ & 6 & 7 & 21 & 2 & $95.1 \%$ \\
\hline $\mathrm{C} 23$ & 11 & 7 & 4 & 1 & $90.1 \%$ \\
\hline $\mathrm{C} 24$ & 8 & 7 & 13 & 1 & $93.0 \%$ \\
\hline $\mathrm{C} 25$ & 9 & 8 & 6 & 1 & $91.4 \%$ \\
\hline $\mathrm{C} 26$ & 6 & 1 & 30 & 1 & $97.5 \%$ \\
\hline $\mathrm{C} 27$ & 5 & 2 & 32 & 1 & $98.2 \%$ \\
\hline $\mathrm{C} 28$ & 5 & $\overline{7}$ & 19 & 2 & $95.5 \%$ \\
\hline $\mathrm{C} 29$ & 6 & 11 & 14 & 2 & $90.8 \%$ \\
\hline C30 & 4 & 7 & 27 & 2 & $96.3 \%$ \\
\hline C31-C32 & 6 & 4 & 19 & 1 & $96.5 \%$ \\
\hline C33 & 2 & 2 & 27 & 1 & $98.9 \%$ \\
\hline D35 & 13 & 6 & 6 & 1 & $88.4 \%$ \\
\hline E36 & 4 & 1 & 24 & 1 & $98.3 \%$ \\
\hline E37-E39 & 2 & 3 & 22 & 2 & $98.3 \%$ \\
\hline $\mathrm{F}$ & 11 & 8 & 0 & 1 & $89.2 \%$ \\
\hline G45 & 4 & 6 & 18 & 2 & $96.4 \%$ \\
\hline G46 & 12 & 8 & 1 & 1 & $88.1 \%$ \\
\hline G47 & 11 & 9 & 0 & 1 & $88.3 \%$ \\
\hline H49 & 11 & 8 & 2 & 1 & $89.3 \%$ \\
\hline H50 & 8 & 3 & 23 & 1 & $95.6 \%$ \\
\hline H51 & 7 & 7 & 11 & 1 & $94.0 \%$ \\
\hline $\mathrm{H} 52$ & 11 & 8 & 2 & 1 & $89.3 \%$ \\
\hline H53 & 6 & 6 & 21 & 1 & $95.7 \%$ \\
\hline I & 11 & 6 & 2 & 1 & $90.6 \%$ \\
\hline J58 & 6 & 6 & 7 & 1 & $95.0 \%$ \\
\hline J59-J60 & 7 & 5 & 11 & 1 & $95.1 \%$ \\
\hline J61 & 10 & 7 & 9 & 1 & $91.4 \%$ \\
\hline J62-J63 & 9 & 8 & 7 & 1 & $91.5 \%$ \\
\hline K64 & 11 & 10 & 0 & 1 & $87.3 \%$ \\
\hline K65 & 9 & 10 & 7 & 2 & $89.7 \%$ \\
\hline K66 & 6 & 9 & 12 & 2 & $93.1 \%$ \\
\hline L68 & 11 & 8 & 1 & 1 & $89.2 \%$ \\
\hline M69-M70 & 10 & 7 & 10 & 1 & $91.4 \%$ \\
\hline M71 & 8 & 7 & 15 & 1 & $93.4 \%$ \\
\hline M72 & 2 & 4 & 30 & 2 & $98.6 \%$ \\
\hline M73 & 6 & 8 & 13 & 2 & $94.0 \%$ \\
\hline M74-M75 & 6 & 2 & 23 & 1 & $97.3 \%$ \\
\hline $\mathrm{N}$ & 8 & 9 & 2 & 2 & $91.1 \%$ \\
\hline O84 & 10 & 8 & 2 & 1 & $90.3 \%$ \\
\hline P85 & 9 & 7 & 8 & 1 & $92.3 \%$ \\
\hline $\mathrm{Q}$ & 8 & 4 & 5 & 1 & $94.5 \%$ \\
\hline R-S & 10 & 7 & 4 & 1 & $91.1 \%$ \\
\hline $\mathrm{T}$ & 1 & 0 & 40 & $1,7,10,16$ & $99.8 \%$ \\
\hline U & 0 & 0 & 44 & & $100.0 \%$ \\
\hline
\end{tabular}

Table D.2: For each sector, the Table reports the number of countries that belong to the two largest communities (1 and 2, respectively), the number of countries that does not belong to any community (i.e. isolated), the indication of the community with the highest number of countries of that sector and the Gini heterogeneity index to measure the dispersion of countries along the communities in the same sector. 


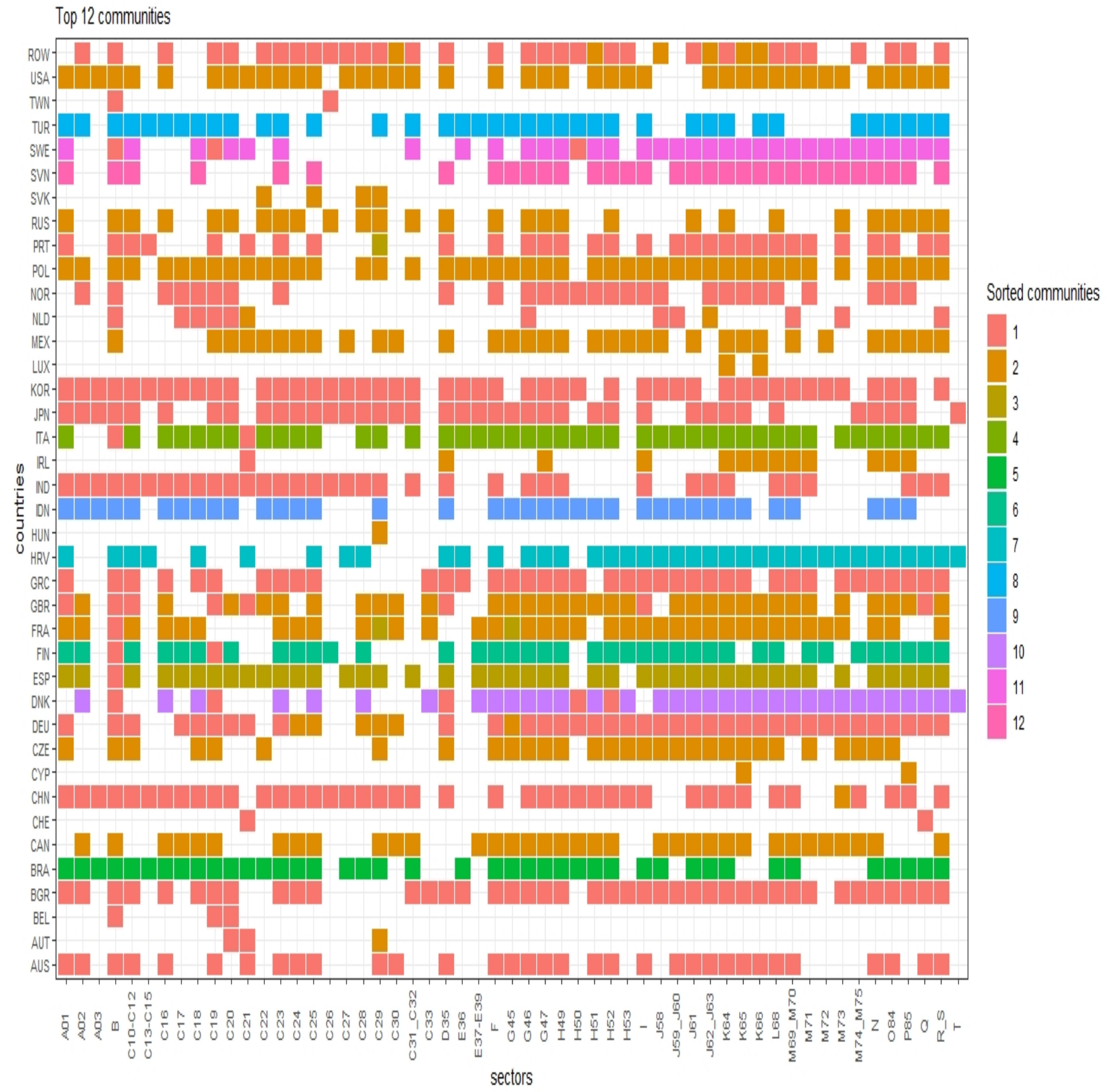

Figure D.1: Membership (in terms of countries and sectors) of larger communities with at least 30 nodes. The communities have been sorted according to the number of nodes. Empty cells represent nodes that belong to smaller communities (lower than 30 nodes) or isolated nodes. The lists of sector names and country names are shown in Tables B.1 and B.2 


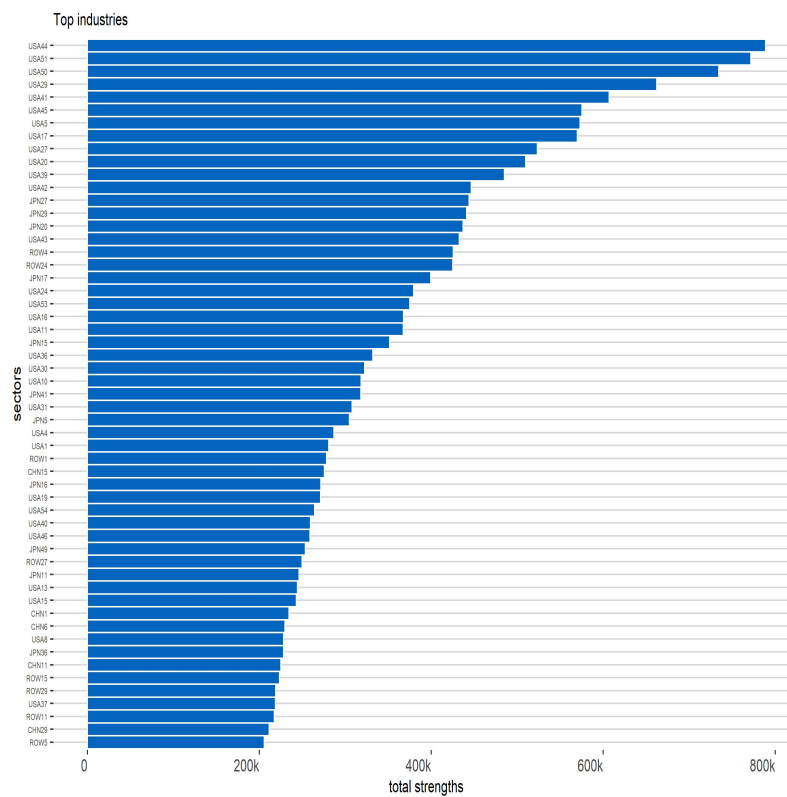

(a) Top industries in Community 1, 2000

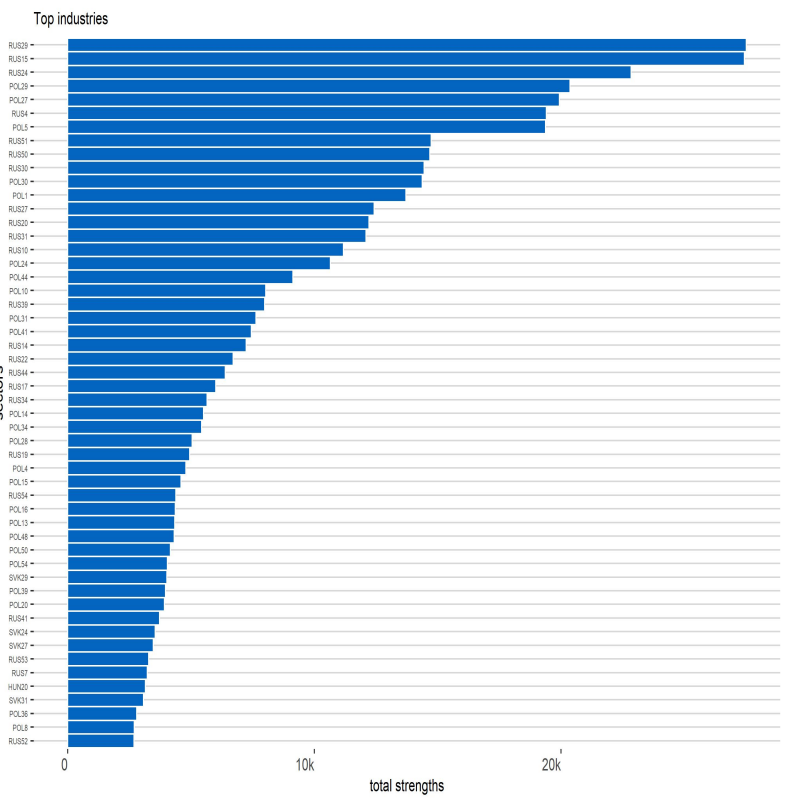

(b) Top industries in Community 2, 2000

Figure D.2: Ranking of top industries in two important international communities in 2000, based on the (total) node strengths.

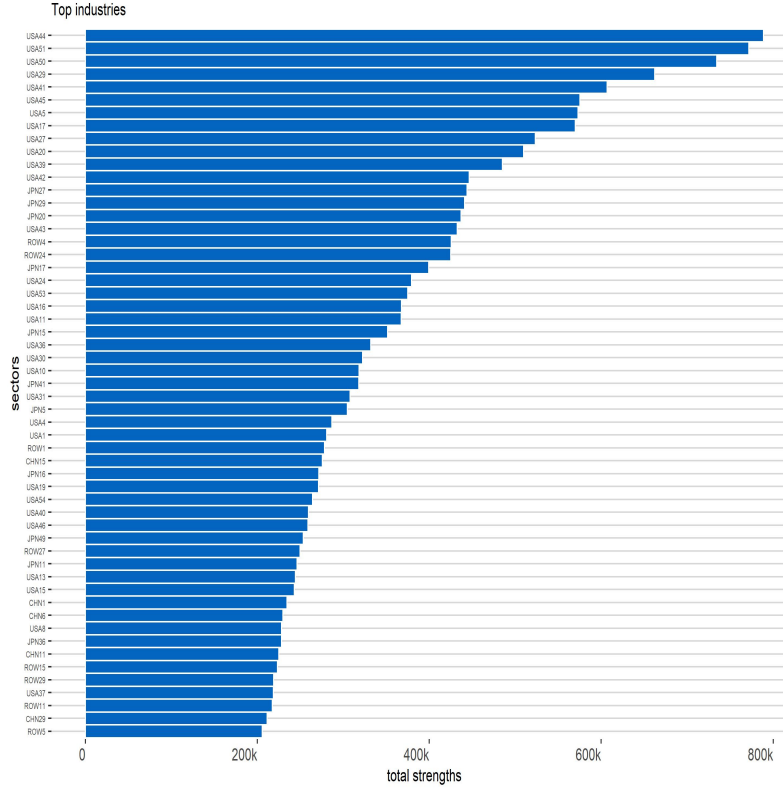

(a) Top industries in Community 1, 2002

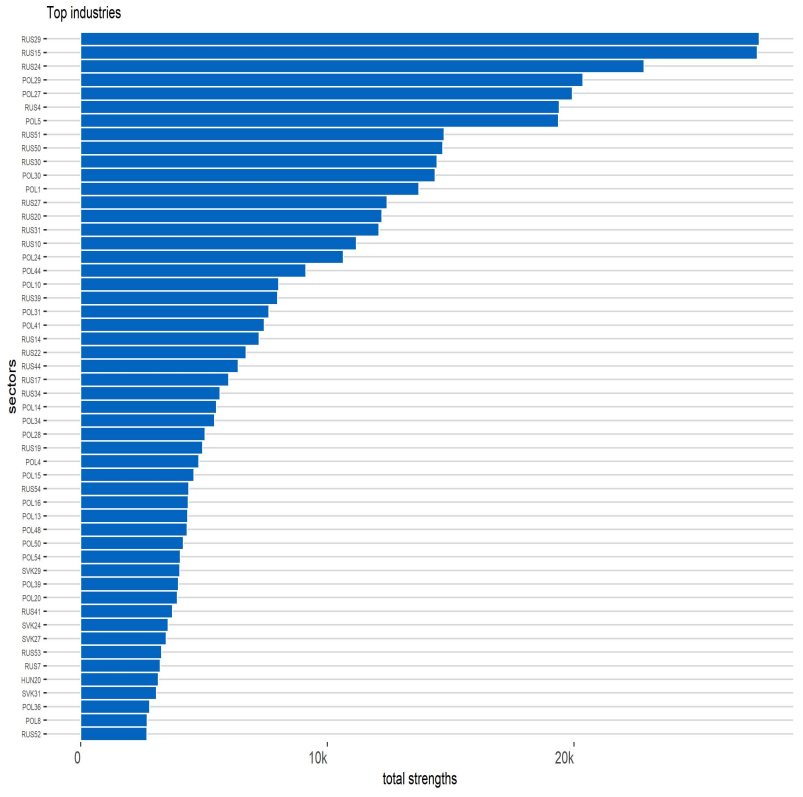

(b) Top industries in Community 2, 2002

Figure D.3: Ranking of top industries in two important international communities in 2002, based on the (total) node strengths. 


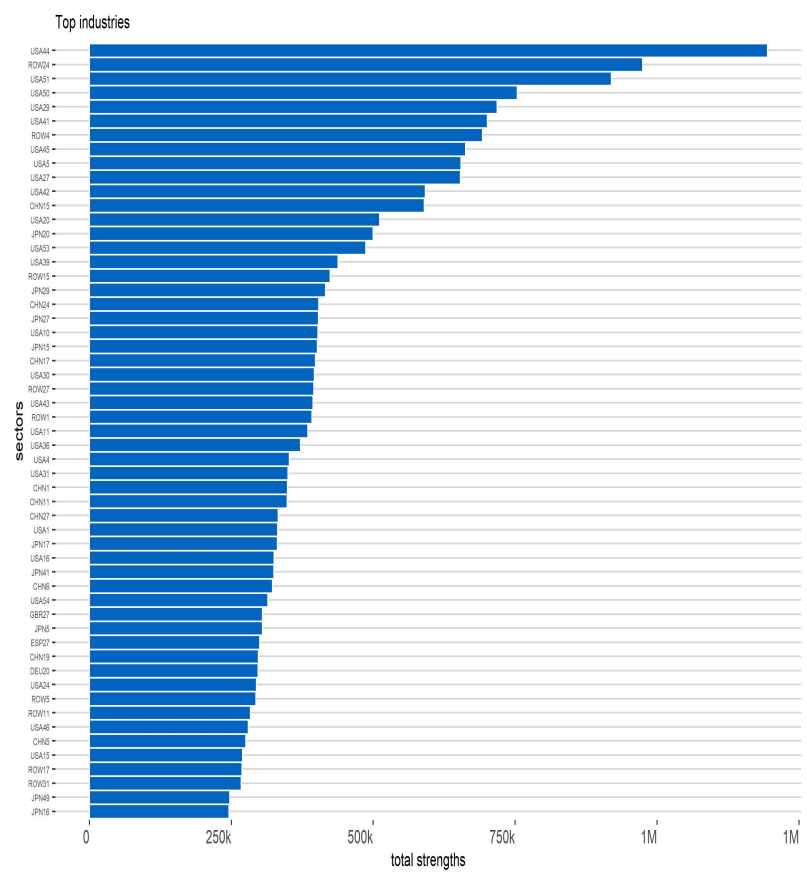

(a) Top industries in Community 1, 2004

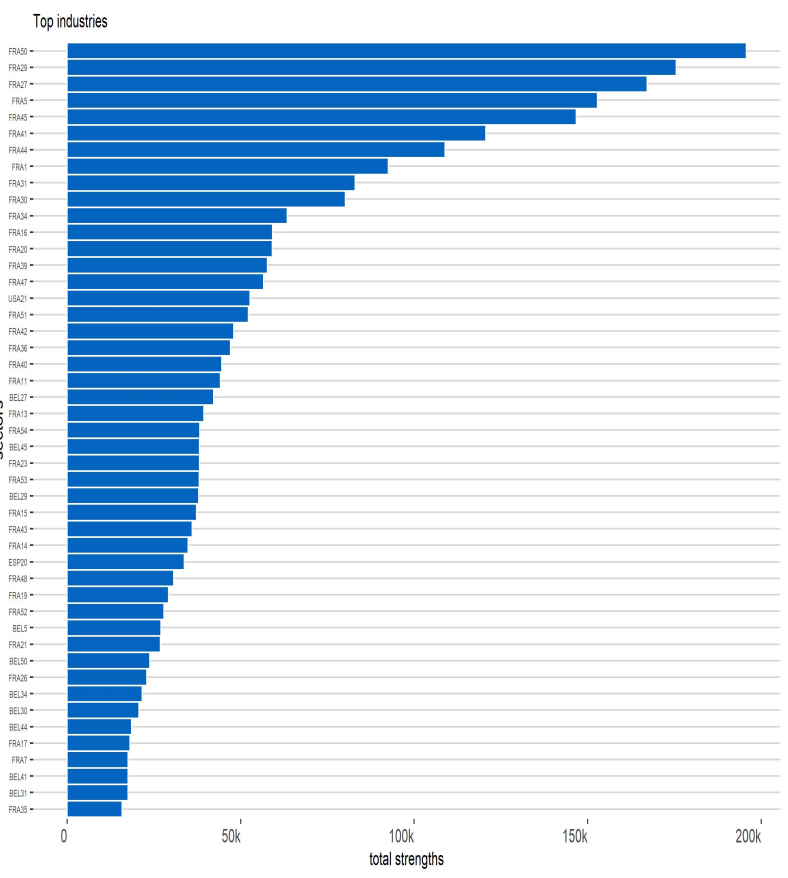

(b) Top industries in Community 2, 2004

Figure D.4: Ranking of top industries in two important international communities in 2004, based on the (total) node strengths.

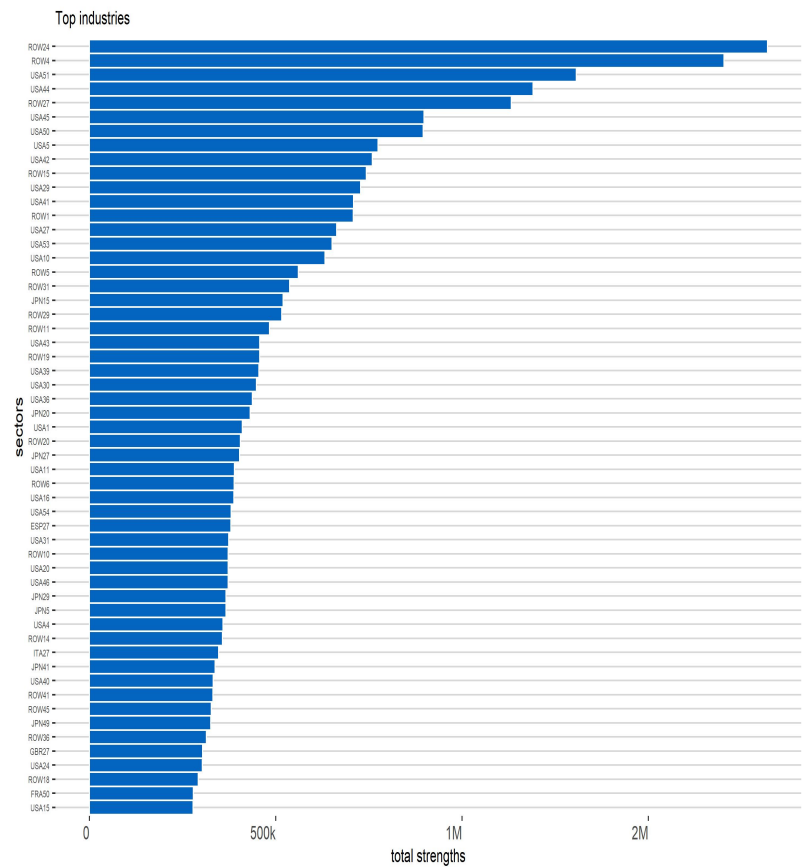

(a) Top industries in Community 1, 2009

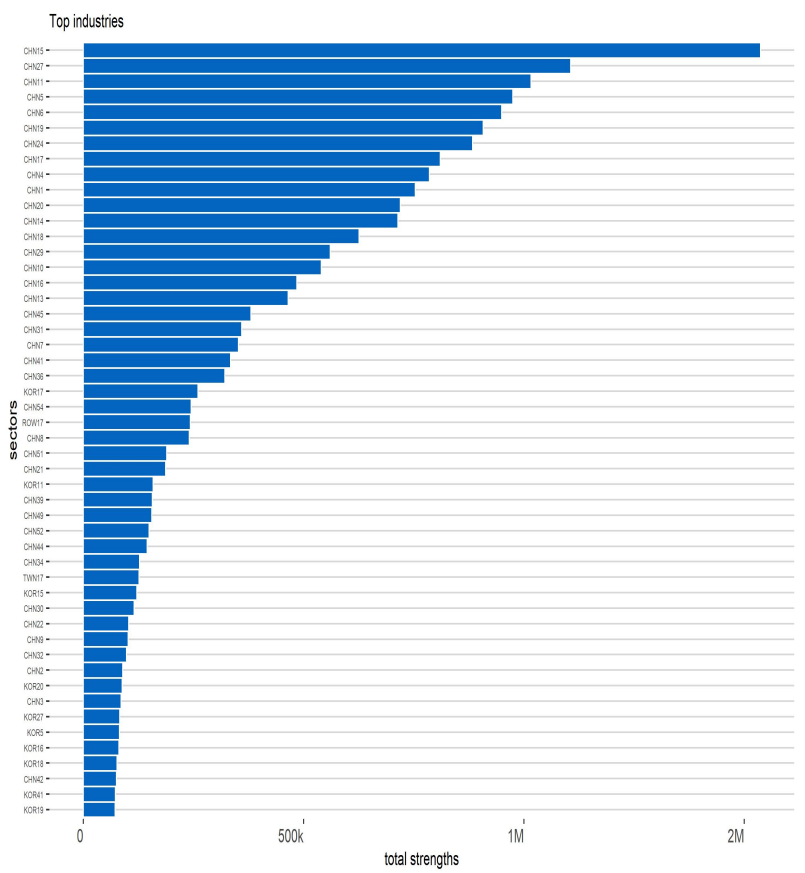

(b) Top industries in Community 2, 2009

Figure D.5: Ranking of top industries in two important international communities in 2009, based on the (total) node strengths. 